Malene Jørgensen, Guy Bauw, and Karen G. Welinder.

\title{
Molecular Properties and Activities of Tuber Proteins of Starch Potato cv. Kuras
}

Supplementary Table S1. Identification of Potato Tuber Proteins Separated by Gelfiltration and SDSPAGE by Tryptic Mass Fingerprinting. Monoisotopic mass, 'exp', experimental; 'cal', calculated; 'ppm', mass accuracy cut-off at $0.5 \mathrm{Da}$; 'protein', indicates assigned protein if more than one is present; 'notes', V, assigned by VEMS only; M, assigned by Mascot only; O, oxygen; C-term, Cterminus of the mature protein; $\mathrm{N}$-term, $\mathrm{N}$-terminus of mature protein; unique: protein variant specific peptide.

TC refers to TIGR contig (http://www.tigr.org/tigr-scripts/tgi/T_index.cgi?species=potato), K to Kuras contig (http://www.bio.aau.dk/en/st-data.htm), and others are GenBank accessions. GenBank accessions DQ and BG are full-length cDNA clones from Kuras.

In the sequences in red bold shows identified peptides and underlined refers to unique peptides.

\section{Fraction I}

Mr SDS PAGE : $\quad 105 \mathrm{kDa}$

Identified protein(s) : Alpha-1,4 glucan phosphorylase L-1 (TC119041)

\begin{tabular}{|c|c|c|c|c|c|}
\hline $\exp$ & cal & ppm & seq & protein & notes \\
\hline 1080.406 & 1080.54 & 121 & YWIGGEDIK & & \\
\hline 1097.384 & 1097.57 & 173 & AFFATAQSVR & & \\
\hline 1120.231 & 1120.60 & 33 & LEEKLTTMR & & \\
\hline 1205.449 & 1205.62 & 142 & IKWEEFPEK & & \\
\hline 1234.430 & 1234.61 & 146 & WTGTEDWVLK & & \\
\hline 1292.545 & 1292.69 & 111 & AVAYDVPIPGYK & & \\
\hline 1335.577 & 1335.78 & 151 & QLLNIFGIVYR & & \\
\hline 1394.571 & 1394.70 & 89 & FVPDERFEEVK & & \\
\hline 1424.582 & 1424.73 & 102 & FIHFTSRNTSSK & & \\
\hline 1536.562 & 1536.67 & 71 & FADNEDLQNEWR & & \\
\hline 1579.615 & 1579.75 & 88 & YHAEFTPVFSPER & & \\
\hline 1601.641 & 1601.78 & 88 & GLNWNEAWNITQR & & \\
\hline 1785.811 & 1785.94 & 71 & TVAYTNHTVLPEALEK & & $\mathrm{V}$ \\
\hline 1785.811 & 1785.86 & 27 & TGYSVVPDAMFDIQVK & & $\mathrm{O}, \mathrm{M}$ \\
\hline 2168.070 & 2168.07 & 2 & VAVQMNDTHPTLCIPELMR & & \\
\hline 2168.070 & 2168.12 & 25 & FITDVGATINHDPEIGDLLK & & \\
\hline 2463.301 & 2463.19 & 44 & EEVGEENFFLFGAQAHEIAGLR & & V \\
\hline 2463.301 & 2463.24 & 25 & MANLCVVGGHAVNGVAEIHSEIVK & & $\mathrm{O}, \mathrm{M}$ \\
\hline 2503.277 & 2503.23 & 19 & NLGHNLENVASQEPDAALGNGGLGR & & \\
\hline 2525.150 & 2525.12 & 11 & SGAFGSYNYDDLIGSLEGNEGFGR & & \\
\hline 2995.746 & 2995.46 & 94 & IHHPITEQGGESDLSSFAPDAASITSSIK & & \\
\hline
\end{tabular}

TC119041, Coverage: $33.7 \%(326 / 966)$

MATANGAHLFNHYSSNSRFIHFTSRNTSSKLFLTKTSHFRRPKRCFHVNNTLSEKIHHPITEQGGESDLSSFAPDAAS ITSSI KYHAEFTPVFSPERFELPKAFFATAQSVRDSLLINWNATYDIYEKLNMKQAYYLSMEFLQGRALLNAIGNLELTGAFAEALKN LGHNLENVASQEPDAALGNGGLGRLASCFLDSLATLNYPAWGYGLRYKYGLFKQRITKDGQEEVAEDWLEIGSPWEVVRNDVS YPIKFYGKVSTGSDGKRYWIGGEDIKAVAYDVPIPGYKTRTTISLRLWSTQVPSADFDLSAFNAGEHTKACEAQANAEKICYI LYPGDESEEGK I LRLKQQYTLCSASLQDI I SRFERRSGDR IKWEEFPEKVAVQMNDTHPTLCI PELMRILIDLKGLNWNEAWN ITQRTVAYTNHTVLPEALEKWSYELMQKLLPRHVE I IEA I DEELVHE IVLKYGSMDLNKLEEKLTTMRI LENFDLPSSVAELF IKPEISVDDDTETVEVHDKVEASDKVVTNDEDDTGKKTSVKIEAAAEKDIDKKTPVSPEPAVIPPKKVRMANLCVVGGHAVNG VAEIHSEIVKEEVFNDFYELWPEKFQNKTNGVTPRRWIRFCNPPLSAI ITKWTGTEDWVLKTEKLAELQKFADNEDLQNEWRE AKRSNKIKVVSFLKEKTGYSVVPDAMFDIQVKRI HEYKRQLLNIFGIVYRYKKMKEMTAAERKTNFVPRVCIFGGKAFATYVQ 
AKRIVKFITDVGATINHDPEIGDLLKVVFVPDYNVSVAELLI PASDLSEH ISTAGMEASGTSNMKFAMNGCIQIGTLDGANVE IREEVGEENFFLFGAQAHEIAGLRKERADGKFVPDERFEEVKEFVRSGAFGSYNYDDLIGSLEGNEGFGRADYFLVGKDFPSY IECQEKVDEAYRDQKRWTTMS I LNTAGSYKFSSDRT IHEYAKDIWNIEAVEIA

Mr SDS PAGE : $\quad 62 \mathrm{kDa}$

Identified protein(s) : Unknown (TC127939)

\begin{tabular}{llllll}
\hline exp & cal & ppm & seq & protein & notes \\
\hline 1030.456 & 1030.60 & 140 & NRKLWWK & & $\mathrm{M}$ \\
1067.541 & 1067.55 & 8 & SGHISDPGLGK & & \\
1067.541 & 1067.63 & 83 & QPINLQKAR & $\mathrm{V}$ \\
1136.359 & 1136.69 & 291 & GLHVLNLSRK & \\
1770.790 & 1770.92 & 72 & MTLGSPASVLTHRSADK & \\
1801.776 & 1801.81 & 19 & SSGGEYDQSFNEGIRR & \\
2308.225 & 2308.08 & 64 & GVSGFWPQNQWVAFPEESSR & \\
3196.061 & 3196.51 & 141 & EVLDEFRGVSGFWPQNQWVAFPEESSR & & \\
\hline
\end{tabular}

TC127939, Coverage: $17.8 \%(97 / 546)$

PVKGDTEEVANHESPVKGDLEEVAYEREDEHDESLSMKRDNSDFDLQARVRSSGGEYDQSFNEGIRRNCSFESEMNEQDHNKS EKDAEAVEVVKSGHISDPGLGKVESWAS PKLQRSCS DLAMRDMVNKLSEQLSLSKSKSFDEMRRLAEKMTLGSPASVLTHRSA DKVMLKKHSSSQLLPSRNRKLWWKLFLWSHRNVQGTGGIQQLP ILANTALNQQGGYSSDTLELGKGMDLSNLGSPGSFTAESL SKGRNDKGKEVLDEFRGVSGFWPQNQWVAFPEESSRFMRISEWVNELPSHPPCLIDEHDHVEDEVNISPSPDAGKSPLISPYP NMNVPEEVAHANTVIRS LNSSSTVAHIAGAGLKVI PAISHLCSLRSVNLSGNF IVQITPGSLPKGLHVLNLSRKKIHTMEGLR EVTRLRVLDLSYNRISRIGQGLSNCTLIKELYLAGNKISDIEGLHRLLKLTVLDLSFNKITTTKALGQLVANYNSLLALNLLG NPIQINISDDQLRKAACSLLPKIAYLNKQPINLQKARDVGTEAVAKAA

Mr SDS PAGE : $\quad 58 \mathrm{kDa}$ Identified protein(s) : 26S proteasome regulatory particle triple A ATPase subunit 2 (TC126136)

\begin{tabular}{llllll}
\hline exp & cal & ppm & seq & protein & notes \\
\hline 1071.576 & 1071.61 & 30 & IEFPLPDIK & & \\
1199.638 & 1199.70 & 54 & KIEFPLPDIK & \\
1328.648 & 1328.76 & 82 & IEFPLPDIKTR & \\
1436.784 & 1436.82 & 26 & IESLDPALLRPGR & \\
2131.071 & 2131.09 & 10 & APLESYADIGGLDAQIQEIK & \\
2488.329 & 2488.33 & 1 & EAVELPLTHPELYEDIGIKPPK & \\
\hline
\end{tabular}

TC126136, Coverage: $15.1 \%(67 / 444)$

MGQGTPGGLNRQLPGDRKNDGDKKEKKEEPAAPPARVGRKQRKQKGPEAAARLPTVTPLTKCKLRLLKLERIKDYLLMEEEFV ANQERLKPQEEKTEEDRSKVDDLRGS PMSVGNLEELIDENHA IVSSSVGPEYYVGILSFVDKDQLEPGCAILMHNKVLSVVGL LQDDVDPMVSVMKVEKAPLESYADIGGLDAQIQEIKEAVELPLTHPELYEDIGIKPPKGVILYGEPGTGKTLLAKAVANSTSA TFLRVVGSELIQKYLGDGPKLVRELFRVADDLSPS IVFIDEIDAVGTKRYDAHSGGEREIQRTMLELLNQLDGFDSRGDVKVI LATNKIESLDPALLRPGRIDRKIEFPLPDIKTRRRIFQI HTARMTLSDDVNLEEFVMTKDEFSGADIKAICTEAGLLALRERR MKVTHADFKKAKDKVMFKKKEGVPEGLYM

Mr SDS PAGE : $\quad 37 \mathrm{kDa}$ Identified protein(s) : Respiratory burst oxidase protein F (AB064343)

\begin{tabular}{|c|c|c|c|c|c|}
\hline $\exp$ & cal & ppm & seq & protein & notes \\
\hline 1003.16 & 1003.51 & 55 & NGVDIVSGSR & & \\
\hline 1079.52 & 1079.57 & 49 & SEFAQSIGIK & & \\
\hline 1156.61 & 1156.60 & 8 & THFARPNWK & & \\
\hline 1197.63 & 1197.65 & 19 & FTLATRWFR & & \\
\hline
\end{tabular}




\begin{tabular}{llll}
1284.65 & 1284.69 & 36 & THFARPNWKK \\
1411.52 & 1411.77 & 177 & VRTHFARPNWK \\
1841.86 & 1842.09 & 126 & GPEGITGILMVILLLFR \\
2233.07 & 2232.94 & 55 & MEEHADSISDFSRSSEYSR \\
2746.3 & 2746.55 & 94 & GDCRLYDVLLLVGLGIGATPFISILK \\
2901.21 & 2901.31 & 37 & VEKINHDELFEYSSQINDESFDSR \\
3065.41 & 3065.52 & 37 & SFSGNLTTTTTDANQRGLGGGGLVNSALEAR \\
\hline
\end{tabular}

\begin{abstract}
AB064343, Coverage: $16.8 \%(158 / 944)$
MKPFSKNDRRRWSFDSVSAGKTADGSASTSPGTEYS INGDQEFVEVTIDLQDDDTIVLRSVEPATAINVIGDISDDNTGIMTP VSISRSPTMKRTSSNRFRQFSQELKAEAVAKRKQLSQELKRFSWSRSFSGNLTTTTTDANQRGLGGGGLVNSALEARTLQKSM AQLDRTRSSAQRALRGLRFNSYKQECVDGWNHVQLNFEKFEKNGYIYRSEFAQSIGIKDSKELALELFDALSRRRRLKVEKIN HDELFEYSSQINDESFDSRLQI FFDIVDKNEDGRITEEEVKEI IMLSASANKLSRLKEQAEEYAALIMEELDPERLGYIELWQ LETLLLQKDTYLNCSQALSCTSQALSQNLQGLRGKSRI HRMSSDFVYIMQENWKRIWVLSLWIMIMIGLFLWKFFQYKQKGAF HVMGYCLLTAKGAAGTLQFYMALI PF PVCRNTITWLRS TRLSYCLPLDDN INFHKT IAGA IVVAVILH IGDHLACDFPRIVRA TEYDYNRYLFHYFQTKQPTYFDLVKGPEGITGILMVILLLFRFTLATRWFRRNLVKLPKPFDRLTGFNAFWYSHHLFVIDYIL LILHGIFLYFAKPWYVRTTWMYLAVPVLRDGGERTLRYFRSGSYSVRLLKVAIYPGNVLTLQMSKPTQFRYKSGQYMFVQCPA VSPFEWHPFSITSAPEDDYIS IHIRQLGDWTQELKRVFSEVCEPPVGGKSGLLRPAERRKKIYPLLLIDGPYGAPKGDCRLYD VLLLVGLGIGATPFISILKDLLNNIVKMEEHADSISDFSRSSEYSRGSNGHTPRRKRILKTTNAYFYWVTREQGSFDWFKGVM NEVAELDQRGVIEMHNYLTSVYEEGDARSALITMVQALKHAKNGVDIVSGSRVRTHFARPNWKKEVTKLSSKHCNARTGVFFC EVPVLGKELSKLCNTFNQKGSSKFEFHKEHF
\end{abstract}

Mr SDS PAGE : $\quad 30 \mathrm{kDa}$

Identified protein(s) : 40S ribosomal protein S2 (TC125898)

\begin{tabular}{|c|c|c|c|c|c|}
\hline $\exp$ & cal & ppm & seq & protein & notes \\
\hline 1374.673 & 1374.67 & 0 & TYGFLTPDFWK & & \\
\hline 1423.684 & 1423.69 & 4 & GGGERGGFGRGFGGR & & M \\
\hline 1447.738 & 1447.74 & 4 & VIVYATEEAAPER & & \\
\hline 1474.671 & 1474.74 & 48 & ETEEEKWVPVTK & & \\
\hline 1540.860 & 1540.87 & 9 & SLEQIYLHSLPIK & & \\
\hline 1573.606 & 1573.88 & 174 & EFQIIDTLIGPSLK & & \\
\hline 1668.835 & 1668.86 & 15 & VLQFAGIEDVFTSSR & & \\
\hline 1690.936 & 1691.12 & 109 & GRIILAKLSVIPVRR & & $3 \mathrm{mis}$ \\
\hline 1693.696 & 1693.84 & 85 & GGGERGGFGRGFGGRGGR & & $3 \mathrm{mis}$ \\
\hline 1746.860 & 1746.89 & 17 & VIVYATEEAAPERVEA & & C-term \\
\hline 1796.925 & 1796.95 & 16 & KVLQFAGIEDVFTSSR & & \\
\hline 1818.237 & 1818.09 & 81 & MVPAPRGAGIVAARVPKK & & $3 \mathrm{mis}$ \\
\hline 1867.948 & 1867.96 & 6 & SPFQEYFDILAKPANK & & \\
\hline 2121.000 & 2121.17 & 80 & VPKKVLQFAGIEDVFTSSR & & M \\
\hline
\end{tabular}

TC125898, Coverage: 52.9\% (148/280)

MAERGGGERGGFGRGFGGRGGRGGDRGGRGRGGRRPRRETEEEKWVPVTKLGRLVKDGKIKSLEQIYLHSLPIKEFQIIDTLI GPSLKDEVMKIMPVQKQTRAGQRTRFKAFVVVGDGNGHVGLGVKCSKEVATAIRGRIILAKLSVIPVRRGYWGNKIGKPHTVP CKVTGKCGSVTVRMVPAPRGAGIVAARVPKKVLQFAGIEDVFTSSRGSTKTLGNFVKATFDCLMKTYGFLTPDEWKETRFTKS PFQEYFDILAKPANKVIVYATEEAAPERVEA

Mr SDS PAGE : $\quad 29 \mathrm{kDa}$

Identified protein(s) : Unknown (TC116056)

\begin{tabular}{llllll}
\hline exp & cal & ppm & seq & protein & notes \\
\hline 1039.731 & 1039.53 & 193 & HPFGSPLER & \\
1144.762 & 1144.66 & 89 & DLLRVMRNK & M
\end{tabular}




\begin{tabular}{lllll}
1249.926 & 1249.72 & 165 & FPQLLIEVYK & \\
1453.913 & 1453.79 & 85 & YRFDSIRDLLR & $\mathrm{M}$ \\
1521.102 & 1520.84 & 172 & FDSIRDLLRVMR & $\mathrm{M}$ \\
1723.654 & 1723.91 & 148 & HPFGSPLERDINITK & \\
1903.214 & 1903.01 & 107 & AVKVLAHPFFWTAEMR & \\
1937.124 & 1936.97 & 80 & LSFLRDSSDRVELEDR & $\mathrm{M}$ \\
1966.252 & 1966.05 & 103 & HPFGSPLERDINITKNK & $\mathrm{M}$ \\
2160.124 & 2160.07 & 25 & WDEKMEPPFIKNIGHYR & $\mathrm{M}$ \\
\hline
\end{tabular}

TC116056, Coverage: $38.2 \%(92 / 241)$

MSSLGHHPTGYGSSGWQAPEQLLHGRQTRAIDMFSLGSVLFFCMTGGRHPFGSPLERDINITKNKVDLFLSEH IPEAVDLFSR LLDPNAELRPKAVKVLAHPFFWTAEMRLSFLRDSSDRVELEDRETSSDLLKALEGTAPVALGGKWDEKMEPPFIKNIGHYRRY RFDSIRDLLRVMRNKLNHYRELPTEIQEILGTVPEGFDGYFRRRFPQLLIEVYKVMSEYCKDEACFQKYFTSSVL

Mr SDS PAGE : $\quad 27 \mathrm{kDa}$

Identified protein(s) : 60S ribosomal L13 (TC119161)

Unknown (CV469081)

\begin{tabular}{|c|c|c|c|c|c|}
\hline $\exp$ & cal & ppm & seq & protein & notes \\
\hline 1019.429 & 1019.51 & 75 & TWFNQPAR & $60 \mathrm{~S}$ & \\
\hline 1040.549 & 1040.46 & 86 & GSAPSYTSDR & Unknown & \\
\hline 1147.556 & 1147.60 & 39 & TWFNQPARK & $60 \mathrm{~S}$ & \\
\hline 1147.556 & 1147.68 & 108 & EKYLVVAAVR & Unknown & M \\
\hline 1244.588 & 1244.66 & 57 & SLEGLQTNVQR & $60 \mathrm{~S}$ & \\
\hline 1262.642 & 1262.72 & 63 & LAPTIGIAVDHR & $60 \mathrm{~S}$ & \\
\hline 1292.520 & 1292.78 & 201 & YLVVAAVRFVR & Unknown & \\
\hline 1390.634 & 1390.82 & 131 & KLAPTIGIAVDHR & $60 \mathrm{~S}$ & \\
\hline 1541.634 & 1541.73 & 62 & VKYEQSLEDSGMR & Unknown & \\
\hline 1663.734 & 1663.74 & 1 & MKHNNVIPNGHFKK & $60 \mathrm{~S}$ & $\mathrm{~N}$-term, M \\
\hline 1763.798 & 1763.89 & 52 & SQLAVPKGSAPSYTSDR & Unknown & \\
\hline 2131.028 & 2131.07 & 20 & HWQNYVRTWFNQPARK & $60 \mathrm{~S}$ & M \\
\hline 2188.250 & 2188.27 & 10 & IFPRPTAGSLRPIVHGQTLK & $60 \mathrm{~S}$ & \\
\hline 2188.250 & 2188.24 & 5 & IKCSFLLNNVVDKVLLLTR & Unknown & M \\
\hline 2449.190 & 2449.27 & 33 & FVRTLISRNDEYLMNHIAEK & Unknown & M \\
\hline 2465.251 & 2465.24 & 4 & FVRTLISRNDEYLMVHIAEK & Unknown & $\mathrm{O}, \mathrm{M}$ \\
\hline 2465.251 & 2465.20 & 21 & AGDSSAEELATATQVQGSYLPITR & $60 \mathrm{~S}$ & \\
\hline 2486.272 & 2486.47 & 80 & AVKIFPRPTAGSLRPIVHGQTLK & $60 \mathrm{~S}$ & \\
\hline 2493.269 & 2493.24 & 12 & VKYEQSLEDSGMRSVGNLLDPR & Unknown & M \\
\hline 2794.123 & 2794.43 & 110 & YLVDSFWDELVKFEKFSSINSLK & Unknown & M \\
\hline
\end{tabular}

TC119161, Coverage: $49.0 \%(101 / 206)$

MKHNNVIPNGHFKKHWQNYVRTWFNQPARKTRRRAARQQKAVKIFPRPTAGSLRPIVHGQTLKYNMKVRAGRGFSLEELKAAG I PKKLAPTIGIAVDHRRRNRSLEGLQTNVQRLKTYKAKLVIFPRRAKKVKAGDSSAEELATATQVQGSYLPITREQPAVDFVK VTDEMKSFKAYGKLRIERTNARHMGARLKRAAEAEKEEKK

CV469081, Coverage: $44.6 \%(111 / 249)$

XESENTDGGNVKQSCVKPEILIN ICDLICFCAVHHPYRIKCSFLLNNVVDKVLLLTRRREKYLVVAAVRFVRTLISRNDEYLM NHIAEKNLLKPIVNAFVANGDRYNLINSAVLELFEY IRKDNLKILLKYLVDSFWDELVKFEKFSSINSLKVKYEQSLEDSGMR SVGNLIDPRKRVDERSLEKEEEDYFNEESDEEDSASASVVNASRVKSQLAVPKGSAPSYTSDRSGGVVDSGDDDNDEDYKPXX

Mr SDS PAGE : $\quad 16 \mathrm{kDa}$

Identified protein(s) : Unknown (CN215334) 


\begin{tabular}{llllll}
\hline exp & cal & ppm & seq & protein & notes \\
\hline 1122.4149 & 1122.60 & 166 & MLFYPNLPK & & \\
1407.5233 & 1407.74 & 155 & MIGSDFLEARIR & \\
1454.5346 & 1454.76 & 158 & TPDVTELAPWVAR & \\
1466.7348 & 1466.70 & 25 & NKDTVQEMLASCK & \\
1915.9731 & 1915.88 & 47 & RMNGGEDWLPFCIYSK & \\
2023.8867 & 2024.06 & 87 & DIPGIHIPSLELACKDFR & \\
2072.0083 & 2071.98 & 14 & RRMNGGEDWLPFCIYSK & $\mathrm{M}$ \\
2721.2934 & 2721.20 & 33 & ACKWPDGLTNGDNSLATHFDAMNDK & \\
\hline
\end{tabular}

CN215334, Coverage: $42.8 \%(107 / 229)$

MSETWEWKLILVS LMVWQI I L LFSWYHESFDREKDIPGIHIPSLELACKDFRACKWPDGLTNGDNSLATHFDAMNDKNKDTVQ EMLASCKQI I S FSHF I PRLELCPEKRMLFYPNLPKMIGSDFLEARIRY I HGARGS TNACH I FGHTHFCWDVLLDG IRYVQAPL AYPRERKRRMNGGEDWLPFCIYSKGELTENMSPCYWSDYYASNPRTPDVTELAPWVARFYRKL

Mr SDS PAGE : $\quad 15 \mathrm{kDa}$

Identified protein(s) : Unknown (TC131597)

\begin{tabular}{llllll}
\hline exp & cal & ppm & seq & protein & notes \\
\hline 1060.316 & 1060.56 & 233 & LINELESSR & \\
1318.186 & 1318.65 & 350 & EEKTQAIDQEK & \\
1475.590 & 1475.74 & 102 & GDVETNMLLENLK & \\
1716.728 & 1716.93 & 116 & VESLIAELETVKEEK & $\mathrm{V}$ \\
1716.728 & 1716.86 & 76 & HLERSASESLESIMK & \\
1915.832 & 1915.95 & 64 & KAMESLASALHEVSSEAR & \\
2072.054 & 2072.12 & 33 & TQAIDQEKLAAESVQSLLK & \\
2313.926 & 2314.23 & 130 & LLEDLKLEVEALTEELEEAK & \\
2410.215 & 2410.21 & 2 & QLEGSNDLLHDAEAEIASLKEK & \\
\hline
\end{tabular}

TC131597, Coverage: 44.6\% (131/294)

KEDDEKNKLLENLEHEKEALRKGDVETNMLLENLKLANEVLRKDDGEKNKLLENLKLQIEALRKEDSQKNKLLEDLKLEVEAL TEELEEAKSYEEKLVEKEALLEQLNVDLEASRMAESYAHNLVEECQKKVEELEAQCKEARHLERSASESLES IMKQLEGSNDL LHDAEAEIASLKEKVGLLEMSTTRQKGDLEESERRAQVAREEASEMSKKVESLIAELETVKEEKTQAIDQEKIAAESVQSLLK EKNKLINELESSREEEEKSKKAMESLASALHEVSSEAREAKERXX

\section{Fraction II}

Mr SDS PAGE : $\quad 100 \mathrm{kDa}$

Identified protein(s) : Alpha-1,4 glucan phosphorylase L-1 (TC119041)

9-13 Lipoxygenase (9-13 LOX, TC112107)

Lipoxygenase (LOX, TC112595)

9-Lipoxygenase (9-LOX , TC112798)

Lipoxygenase-2 ( LOX-2, TC112465)

\begin{tabular}{lllll}
\hline exp & cal & ppm & seq & protein \\
\hline 858.426 & 858.47 & 54 & LQEFPPK & 9-13 LOX, LOX, 9-LOX, LOX-2 \\
899.473 & 899.53 & 69 & LPQGPLFK & 9-13 LOX, LOX, 9-LOX, LOX-2 \\
914.598 & 914.57 & 29 & FPTPLVIK & 9-13 LOX, LOX, 9-LOX, LOX-2 \\
919.451 & 919.45 & 4 & TDPKSESR & 9-13 LOX, LOX, 9-LOX, LOX-2 \\
945.603 & 945.53 & 77 & LLYPHFR & 9-13 LOX, LOX, 9-LOX, LOX-2 \\
1080.571 & 1080.54 & 33 & YWIGGEDIK & L-1 \\
1097.601 & 1097.57 & 25 & AFFATAQSVR & L-1
\end{tabular}




\begin{tabular}{|c|c|c|c|c|c|}
\hline 1139.791 & 1139.58 & 185 & ELREVGHGDK & 9-13 LOX, LOX, 9-LOX, LOX-2 & \\
\hline 1154.637 & 1154.63 & 5 & AFATYVQAKR & $\mathrm{L}-1$ & \\
\hline 1161.556 & 1161.59 & 29 & DKEPLAAFDK & 9-13 LOX, LOX, 9-LOX, LOX-2 & \\
\hline 1171.649 & 1171.69 & 39 & QLSVLHPIHK & 9-13 LOX, LOX, 9-LOX, LOX-2 & \\
\hline 1189.679 & 1189.56 & 97 & DNELQAWWK & 9-13 LOX, LOX, 9-LOX, LOX-2 & \\
\hline 1205.714 & 1205.62 & 78 & IKWEEFPEK & L-1 & \\
\hline 1234.654 & 1234.61 & 35 & WTGTEDWVLK & L-1 & \\
\hline 1234.654 & 1234.65 & 3 & KVKGTVVMMNK & 9-13 LOX & Unique, $\mathrm{M}$ \\
\hline 1244.687 & 1244.72 & 31 & FPTPLVIKDSK & 9-13 LOX, LOX, 9-LOX, LOX-2 & \\
\hline 1292.695 & 1292.65 & 38 & NMHINEFFLK & 9-13 LOX & Unique \\
\hline 1292.695 & 1292.69 & 4 & AVAYDVPIPGYK & L-1 & \\
\hline 1298.755 & 1298.75 & 4 & ALTAAIPLEMIR & 9-13 LOX, LOX & \\
\hline 1306.656 & 1306.71 & 42 & YRENELLTLR & 9-13 LOX, LOX, 9-LOX, LOX-2 & \\
\hline 1331.788 & 1331.76 & 23 & TPVSPEPAVIPPK & $\mathrm{L}-1$ & \\
\hline 1332.637 & 1332.65 & 13 & HTTDEIYLGQR & 9-13 LOX, LOX, 9-LOX, LOX-2 & \\
\hline 1336.138 & 1335.78 & 270 & QLLNIFGIVYR & L-1 & \\
\hline 1371.700 & 1371.76 & 43 & ELLRTDGEGILR & 9-13 LOX, LOX, 9-LOX, LOX-2 & \\
\hline 1381.612 & 1381.65 & 27 & TAWRTDEEFAR & 9-13 LOX, LOX, 9-LOX, LOX-2 & \\
\hline 1411.735 & 1411.69 & 35 & TTLGGSAEYPYPR & 9-13 LOX, LOX, 9-LOX & \\
\hline 1434.731 & 1434.81 & 55 & KYRENELLTLR & 9-13 LOX, LOX, 9-LOX, LOX-2 & $\mathrm{V}$ \\
\hline 1452.840 & 1452.83 & 7 & IFFVNQPYLPSK & LOX & Unique \\
\hline 1459.791 & 1459.85 & 41 & KTPVSPEPAVIPPK & L-1 & \\
\hline 1459.791 & 1459.85 & 41 & TPVSPEPAVIPPKK & L-1 & \\
\hline 1459.791 & 1459.78 & 8 & ELFGGHDDSKKVK & 9-LOX & Unique \\
\hline 1536.701 & 1536.67 & 19 & FADNEDLQNEWR & L-1 & \\
\hline 1621.707 & 1621.69 & 10 & QAYYLSMEFLQGR & L-1 & M \\
\hline 1659.951 & 1659.95 & 3 & LYEGGIKLPQGPLFK & 9-13 LOX, LOX, 9-LOX, LOX-2 & \\
\hline 1713.785 & 1713.88 & 58 & DWVFPDQALPADLVK & 9-13 LOX, LOX, 9-LOX, LOX-2 & \\
\hline 1755.931 & 1756.00 & 39 & TDGEGILRFPTPLVIK & 9-13 LOX, LOX, 9-LOX, LOX-2 & \\
\hline 1785.853 & 1785.94 & 48 & TVAYTNHTVLPEALEK & L-1 & \\
\hline 1889.984 & 1890.03 & 26 & QILVNAGGVLESTVFQSK & 9-13 LOX & Unique \\
\hline 1925.927 & 1925.98 & 27 & TGYSVVPDAMFDIQVKR & L-1 & \\
\hline 2007.949 & 2008.00 & 26 & VYTPSDQGVESSIWQLAK & 9-13 LOX, LOX, 9-LOX, LOX-2 & \\
\hline 2018.972 & 2019.02 & 25 & LLYPHFRDTMNINASAR & 9-13 LOX, 9-LOX & \\
\hline 2018.972 & 2018.98 & 4 & ESPEWTKDKEPLAAFDR & 9-13 LOX & Unique, $\mathrm{M}$ \\
\hline 2018.972 & 2019.17 & 96 & LFILNHHDVLIPYLRR & LOX, LOX-2 & \\
\hline 2075.059 & 2075.08 & 8 & VSFQLISSVQGDPTNGLQGK & 9-13 LOX, 9-LOX, LOX & \\
\hline 2135.097 & 2135.12 & 9 & IFFANQPYLPSETPELLR & 9-13 LOX, LOX-2 & \\
\hline 2135.097 & 2135.09 & 3 & VSFQLISSVQSDPANGLQGK & LOX-2 & Unique \\
\hline 2168.100 & 2168.07 & 16 & VAVQMNDTHPTLCIPELMR & L-1 & $\mathrm{V}$ \\
\hline 2168.100 & 2168.12 & 11 & FITDVGATINHDPEIGDLLK & L-1 & \\
\hline 2206.152 & 2206.14 & 6 & SGPVNAPYTLLFPTSEGGLTGK & 9-13 LOX, LOX, 9-LOX, LOX-2 & \\
\hline 2228.131 & 2228.22 & 39 & HVEIIEAIDEELVHEIVLK & L-1 & \\
\hline 2263.148 & 2263.21 & 28 & IFFANQPYLPSETPELLRK & 9-13 LOX, LOX-2 & \\
\hline 2263.148 & 2263.14 & 4 & TDEEFAREMLAGVNPVIISR & LOX & Unique, $\mathrm{O}$ \\
\hline 2277.205 & 2277.19 & 7 & TDEEFAREMLAGVNPIIISR & 9-13 LOX, 9-LOX, LOX-2, & \\
\hline 2308.168 & 2308.21 & 19 & LLIEDYPYAVDGLEIWSAIK & 9-13 LOX, LOX, 9-LOX, LOX-2 & \\
\hline 2345.156 & 2345.09 & 29 & LDPEAYGNQNSTITAEHIEDK & 9-13 LOX, LOX, 9-LOX, LOX-2 & \\
\hline 2501.783 & 2501.16 & 249 & IYDYDIYNDLGNPDQGKENVR & 9-13 LOX, 9-LOX & \\
\hline 2525.173 & 2525.12 & 21 & SGAFGSYNYDDLIGSLEGNEGFGR & L-1 & \\
\hline
\end{tabular}

TC119041, Coverage: $23.4 \%$ (238 / 966) 
MATANGAHLFNHYSSNSRFIHFTSRNTSSKLFLTKTSHFRRPKRCFHVNNTLSEKIHHP ITEQGGESDLSSFAPDAASITSS I KYHAEFTPVFSPERFELPKAFFATAQSVRDSLLINWNATYDIYEKLNMKQAYYLSMEFLQGRALLNAIGNLELTGAFAEALKN LGHNLENVASQEPDAALGNGGLGRLASCFLDSLATLNYPAWGYGLRYKYGLFKQRITKDGQEEVAEDWLEIGSPWEVVRNDVS YPIKFYGKVSTGSDGKRYWIGGEDIKAVAYDVPIPGYKTRTTISLRLWSTQVPSADFDLSAFNAGEHTKACEAQANAEKICYI LYPGDESEEGKI LRLKQQYTLCSASLQDI I SRFERRSGDRIKWEEFPEKVAVQMNDTHPTLCIPELMRILIDLKGLNWNEAWN ITQRTVAYTNHTVLPEALEKWSYELMQKLLPRHVEI IEAIDEELVHEIVLKYGSMDLNKLEEKLTTMRI LENEDLPSSVAELF IKPEISVDDDTETVEVHDKVEASDKVVTNDEDDTGKKTSVKIEAAAEKDIDKKTPVSPEPAVIPPKKVRMANLCVVGGHAVNG VAEIHSEIVKEEVFNDFYELWPEKFQNKTNGVTPRRWIRFCNPPLSAI ITKWTGTEDWVLKTEKLAELQKFADNEDLQNEWRE AKRSNKIKVVSFLKEKTGYSVVPDAMFDIQVKRI HEYKRQLLNIFGIVYRYKKMKEMTAAERKTNFVPRVCIFGGKAFATYVQ AKRIVKFITDVGATINHDPEIGDLLKVVFVPDYNVSVAELLI PASDLSEH ISTAGMEASGTSNMKFAMNGCIQIGTLDGANVE IREEVGEENFFLFGAQAHEIAGLRKERADGKFVPDERFEEVKEFVRSGAFGSYNYDDLIGSLEGNEGFGRADYFLVGKDFPSY IECQEKVDEAYRDQKRWTTMS ILNTAGSYKFSSDRTIHEYAKDIWNIEAVEIA

TC112107, Coverage: 44.3\% (381/861)

MIGQITSGLFGGHDDSKKVKGTVVMMNKNVLDFTDLASSLTGKIFDVLGQKVSFQLISSVQGDPTNGLQGKHSNPAYLENSLF TLTPLTAGSETAFGVTF DWNEEFGVPGA I I KNMH INEFFLKSLTLEDVPNHGKVHFVCNSWVYPSLNYKSDRIFFANQPYLP SETPELLRKYRENELLTLRGDGTGKREAWDRIYDYDIYNDLGNPDQGKENVRTTLGGSAEYPYPRRGRTGRPPTRTDPKSESR IPLILSLDIYVPRDERFGHLKMS DFLTYALKS IVQF ILPELHALFDGTPNEFDSFEDVLRLYEGGIKLPQGPLFKALTAAIPL EMIRELLRTDGEGILRFPTPLVIKDSKTAWRTDEEFAREMLAGVNPIIISRLQEFPPKSKLDPEAYGNQNSTITAEHIEDKLD GLTVDEAMNNNKLFILNHHDVI I PYLRRINTTITKTYASRTLLFLQDNGSLKPLAIELSLPHPDGDQFGVTSKVYTPSDQGVE SSIWQLAKAYVAVNDTGVHQL ISHWLNTHAVIE PFVIATNRQLSVLHPIHKLLYPHFRDTMNINASARQILVNAGGVLESTVF QSKFAMEMSAVVYKDWVFPDQALPADLVKRGVAVEDSSSPHGVRLLIEDYPYAVDGLEIWSAIKSWVTDYCSFYYGSDEEILK DNELQAWWKELREVGHGDKKNEPWWPEMKTPQELI DSCTTI IWIASALHAAVNFGQYPYAGYLPNRPTVSRRFMPEPGTPEYE ELKRNPDKAFLKT ITAQLQTLLGVSLVE ILSRHTTDE IYLGQRESPEWTKDKEPLAAFDRFGKKLTDIEKQ I IQRNGDN ILTN RSGPVNAPYTLLFPTSEGGLTGKGIPNSVSI

TC112595, Coverage: $36.5 \%(314 / 860)$

MIGQIKSGLFGPPDDSKKLKGTVVMMNKNALGFTDLAGSLTDTAFDVLGQKVSFQLISSVQGDPTNGLQGKHSNPAYLENSLF TLPPLTVDETSFGVTFDWNEEFGVPGAFI I KNTH INEFFLKSLTLEDVPNHGKVHFVCNSWVYPSFSYKSDRIFFVNQPYLPS KTPELLRKYRENELLTLRGDGTGKREAWDRIYDYDIYNDLGNPDEGKENVRTTLGGSAEYPYPRRGRTGRPPTRTDPKSESRI PLILSLDIYVPRDERFGHLKMSDFLTYALKS IVQF I LPELHALFDGTPNEFDSFEDVLRLYEGGIKLPQGPLFKALTAAIPLE MIRELLRTDGEGILRFPTPLVIKDSKTAWRTDEEFAREMLAGVNPVIISRLQEFPPKSKLDPEAYGNQNSTITAEHIEDKLDG LTVDEAMNNNKLFILNHHDVLIPYLRRINTTTTKTYASRTLLFLQDNGSLKPLAIELSLPHPDGDQFGVTSKVYTPSDQGVES SIWQLAKAYVAVNDS GVHQLI S HWLNTHAVIEPFVIATNRQLSVLHPIHKLLYPHFRDTMN INAMARQILINAGGVLESTVFP SKFAMEMSAVVYKDWVFPDQALPADLVKRGVAVEDSSSPHGVRLLIEDYPYAVDGLEIWSAIKSWVTDYCSFYYGSDEEILKD NELQAWWKELREVGHGDKKNEPWWPEMERPQEL I DSTT I IWIASALHAAVNFGQYPYAGYLPNRPTVSRRFMPEPGTPEYEE LKKNPDKAFLKTITAQLQTLLGVSLIEILSRHTTDEIYLGQRESPEWTKDKEPLAAFDKFGKKLTDIEKQ I IQRNGDN ILTNR SGPVNAPYTLLFPTSEGGLTGKGIPNSVSI

TC112798, Coverage: $38.7 \%(334 / 864)$

MNIGQ IMGGRELFGGHDDSKKVKGTVVMMKKNALDFTDLAGSLTDKAFDVLGQKVSFQLISSVQGDPTNGLQGKHSNPAYLEN SLFTLTPLTAGSETAFGVTFDWNEEFGVPGAFI IKNTH INEFFLKSLTLEDVPNHGKVHFDCNSWVYPSFRYKSDRIFEANQP YLPSKTPELLRKYRENELLTLRGDGTGKREAWDRIYDYDIYNDLGNPDQGKENVRTTLGGSAEYPYPRRGRTGRPPTRTDPKS ESRI PLLLSLDIYVPRDERFGHLKMS DFLTYALKS IVQF I LPELHALFDGT PNEFDSFEDVLRLYEGGIKLPQGPLFKALTAA I PLEMIKELLRTDGEGILRFPTPLVIKDSKTAWRTDEEFAREMLAGVNPIIISRLQEFPPKSKLDPEAYGNQNSTITAEHIED KLDGLTVDEAMNNNKLFILNHHDLLIPYLRRINTTITKSYASRTLLFLQDNGSLKPLAIELSLPHPDGDQFGVTSKVYTPSDQ GVESSIWQLAKAYVAVNDAGVHQL I SHWLNTHAVIEPFVIATNRQLSVLHPIHKLLYPHFRDTMNINASARQ I LINAGGVLES TVFQSKFALEMSAVVYKDWVFPDQALPADLVKRGVAVEDSSSPHGVRLLIEDYPYAVDGLEIWSAIKSWVTDYCSFYYGSDEE I LKDNELQAWWKELREVGHGDKKNEPWWPEMETPQELIDSCTTI IWIASALHAAVNFGQYPYAGYLPNRPTVSRRFMPEPGTP EYEELKKNPDKAFLKTITAQLQTLLGVSLVEILSRHTTDEIYLGQRESPEWTKDKEPLAAFDKFGKKLTDIEKQI IQRNGDNI LTNRSGPVNAPYTLLFPTSEGGLTGKGIPNSVS I

TC112465, Coverage: 32.4\% (279 / 860)

MLGQIVGGLIGGHHDSKKVKGTVVMMKKNALDFTDLAGSLTDKIFEALGQKVSFQLISSVQSDPANGLQGKHSNPAYLENFLF TLTPLAAGETAFGVTFDWNEEFGVPGAFI I KNTH INEFFLKSLTLEDVPNHGKVHFVCNSWVYPSERYKSDRIFFANQPYLPS ETPELLRKYRENELITLRGDGTGKREAWDRIYDYDVYNDLGNPDQGEQNVRTTLGGSADYPYPRRGRTGRPPTRTDPKSESRI PLILSLDIYVPRDERFGHLKMSDFLTYALKS IVQFILPELHALFDGTPNEFDSFEDVLRLYEGGIKLPQGPLFKALTAAIPLE MMKELLRTDGEGILRFPTPLVIKDSKTAWRTDEEFAREMLAGVNPIIISRLQEFPPKSKLDPEAYGNQNSTITAEHIEDKLDG 
LTVDEAMNNNKLF ILNHHDVLI PYLRRINTTTTKTYASRTLLFLQDNGSLKPLAIELSLPHPDGDQFGVTSKVYTPSDQGVES SIWQLAKAYVAVNDSGVHQLISHWLNTHAV IEP FVIATNRQLSVLHPIHKLLYPHFRDTMN INAMARQIL INAGGVLESTVEP SKFAMEMSAVVYKDWVFPDQALPADLVKRGVAVEDSSSPHGVRLLIEDYPYAVDGLEIWSAIKSWVTDYCSFYYGSDEEILKD NELQAWWKELREVGHGDKKNEPWWPEMETPQEL I DSCTT I IWIASALHAAVNFGQYPYAGYLPNRPTVSRRFMPEPGTPEYEE LKKNPDKAFLKTITAQLQTLLGVSLIEI LSRHTTDE IYLGQRESPEWTKDKEPLAAFDKFGKKLTDIEKQ I I QRNGDNILTNR SGPVNAPYTLLFPTSEGGLTGKGI PNSVS I

Mr SDS PAGE : $\quad 95 \mathrm{kDa}$

Identified protein(s) : Lipoxygenase -2 (TC112465)

\begin{tabular}{|c|c|c|c|c|c|}
\hline $\exp$ & cal & ppm & seq & protein & notes \\
\hline 1133.680 & 1133.58 & 85 & DKEPLAAFDK & & \\
\hline 1189.667 & 1189.56 & 87 & DNELQAWWK & & \\
\hline 1298.769 & 1298.75 & 15 & ALTAAIPLEMIR & & \\
\hline 1398.753 & 1398.78 & 17 & EMLAGVNPVIISR & & \\
\hline 1511.792 & 1511.92 & 84 & IPLILSLDIYVPR & & \\
\hline 1713.759 & 1713.88 & 73 & DWVFPDQALPADLVK & & \\
\hline 1862.995 & 1863.06 & 37 & LFILNHHDVLIPYLR & & \\
\hline 1869.911 & 1869.99 & 40 & DWVFPDQALPADLVKR & & \\
\hline 1873.039 & 1873.04 & 2 & QILINAGGVLESTVFPSK & & \\
\hline 2007.997 & 2008.00 & 3 & VYTPSDQGVESSIWQLAK & & \\
\hline 2247.192 & 2247.14 & 21 & TDEEFAREMLAGVNPVIISR & & \\
\hline 2308.283 & 2308.21 & 31 & LLIEDYPYAVDGLEIWSAIK & & \\
\hline 2502.175 & 2502.14 & 13 & IYDYDIYNDLGNPDEGKENVR & & \\
\hline 2690.490 & 2690.58 & 33 & LPQGPLFKALTAAIPLEMIRELLR & & M \\
\hline
\end{tabular}

TC112595, Coverage: $21.4 \%(184 / 860)$

MIGQIKSGLFGPPDDSKKLKGTVVMMNKNALGFTDLAGSLTDTAFDVLGQKVSFQLISSVQGDPTNGLQGKHSNPAYLENSLF TLPPLTVDETSFGVTFDWNEEFGVPGAFI IKNTH INEFFLKSLTLEDVPNHGKVHFVCNSWVYPSFSYKSDRIFFVNQPYLPS KTPELLRKYRENELLTLRGDGTGKREAWDRIYDYDIYNDLGNPDEGKENVRTTLGGSAEYPYPRRGRTGRPPTRTDPKSESRI PLILSLDIYVPRDERFGHLKMSDFLTYALKS IVQF I LPELHALFDGTPNEFDSFEDVLRLYEGGIKLPQGPLFKALTAAIPLE MIRELLRTDGEGILRFPTPLVIKDSKTAWRTDEEFAREMLAGVNPVIISRLQEFP PKSKLDPEAYGNQNSTITAEHIEDKLDG LTVDEAMNNNKLFILNHHDVLIPYLRRINTTTTKTYASRTLLFLQDNGSLKPLAIELSLPHPDGDQFGVTSKVYTPSDQGVES SIWQLAKAYVAVNDS GVHQLIS HWLNTHAVIEPFVIATNRQLSVLHP I HKLLYPHFRDTMNINAMARQILINAGGVLESTVFP SKEAMEMSAVVYKDWVFPDQALPADLVKRGVAVEDSSSPHGVRLLIEDYPYAVDGLEIWSAIKSWVTDYCSFYYGSDEEILKD NELQAWWKELREVGHGDKKNEPWWPEMERPQELIDSCTTI IWIASALHAAVNFGQYPYAGYLPNRPTVSRRFMPEPGTPEYEE LKKNPDKAFLKTITAQLQTLLGVSLIEILSRHTTDE IYLGQRES PEWTKDKEPLAAFDKFGKKLTDIEKQ I IQRNGDNILTNR SGPVNAPYTLLFPTSEGGLTGKGIPNSVSI

Mr SDS PAGE : $\quad 65 \mathrm{kDa}$

Identified protein(s) : Cpn 60 alpha subunit (TC112135)

\begin{tabular}{|c|c|c|c|c|c|}
\hline $\exp$ & cal & ppm & seq & protein & notes \\
\hline 1017.523 & 1017.56 & 36 & APGFGERRK & & $\mathrm{M}$ \\
\hline 1062.576 & 1062.56 & 17 & LIAEFENAR & & \\
\hline 1121.600 & 1121.63 & 28 & VAAPAAPQGLQV & & C-term \\
\hline 1182.717 & 1182.68 & 28 & LADAVGLTLGPR & & \\
\hline 1234.673 & 1234.63 & 34 & NVVLDEYGTPK & & \\
\hline 1243.715 & 1243.71 & 1 & TVLGLIEELEK & & \\
\hline 1371.778 & 1371.81 & 23 & TVLGLIEELEKK & & \\
\hline 1469.738 & 1469.73 & 7 & GYISPQFVTNSEK & & \\
\hline 1555.892 & 1555.91 & 9 & LGLLSVTSGANPVSLK & & \\
\hline 1561.699 & 1561.69 & 6 & ELFETDSVYDSEK & & \\
\hline 1754.899 & 1754.91 & 7 & AIELPDAMENAGAALIR & & \\
\hline
\end{tabular}




\begin{tabular}{lllll}
1769.231 & 1769.02 & 119 & GILNVAAIKAPGFGERR & M \\
1920.946 & 1920.95 & 3 & DSTTIIADAASKDEIQSR & \\
2120.019 & 2120.08 & 30 & TNDSAGDGTTTASVLAREIIK & \\
2264.254 & 2264.25 & 2 & AIVAPASLIAQNAGVEGEVVVEK & \\
2264.254 & 2264.31 & 25 & APLLIIAEDITGEALATLVVNK & $\mathrm{V}$ \\
2309.314 & 2309.29 & 10 & SRSALQAGIDKLADAVGLTLGPR & $\mathrm{M}$ \\
3000.620 & 3000.58 & 12 & NATFAAIEEGIVPGGGAAFVHLSTYVPAIK & \\
\hline
\end{tabular}

TC112135, Coverage: $43.9 \%(258 / 588)$

MASANA I STAS I I PS P SKQGGLKNRKVSQLQGQRFGNKGAKNRFVVKACAKE IAFDQKSRSALQAGIDKLADAVGLTLGPRGR NVVLDEYGTPKVVNDGVTIARAIELPDAMENAGAALIREVASKTNDSAGDGTTTASVLAREIIKLGLISVTSGANPVSLKRGI DKTVLGLIEELEKKARPVKGRDDIKAIAS I SAGNDES I GTMIADA I DKVGPDGVLS IESSSSLETTVHVEEGMEIDRGYISPQ FVTNSEKLIAEFENARVLVTDQK I SAIKDI I PLLEKTTQLRAPLLI IAEDITGEALATLVVNKLRGILNVAAIKAPGFGERRK ALLQDIAIVTGAEYQATDLGLLVENTPVEALGIARKVTITKDSTTIIADAASKDEIQSRIAQLKKELFETDSVYDSEKLAERI AKLSGGVAVIKVGAATEAELEDRKLRIEDAKNATFAAIEEGIVPGGGAAFVHLSTYVPAIKEKIEDADERLGADI IQKAIVAP ASLIAQNAGVEGEVVVEKVKEAEWEMGYNAMTDIYENLVDAGVIDPAKVIRCALQNSASVAGMVLTTQAIVVEKAKPKVAAPA APQGLQV

Mr SDS PAGE : $\quad 60 \mathrm{kDa}$ Identified protein(s) : Cpn 60 beta subunit (TC119088)

\begin{tabular}{|c|c|c|c|c|c|}
\hline $\exp$ & cal & ppm & seq & protein & notes \\
\hline 1182.719 & 1182.71 & 8 & LADLVGVTLGPK & & \\
\hline 1256.632 & 1256.68 & 42 & DLVNVLEDAIR & & \\
\hline 1280.717 & 1280.77 & 40 & VVAAGANPVLITR & & \\
\hline 1491.638 & 1491.70 & 42 & GYVSPYFVTDSEK & & \\
\hline 1517.792 & 1517.84 & 32 & GRNVVLESKYGAPK & & M \\
\hline 1541.703 & 1541.77 & 43 & EVELEDPVENIGAK & & \\
\hline 1559.728 & 1559.85 & 78 & YGAPKIVNDGVTVAR & & M \\
\hline 1643.753 & 1643.88 & 76 & AAVEEGIVVGGGCTLLR & & \\
\hline 1665.760 & 1665.80 & 22 & NLIEAADQDYEKEK & & \\
\hline 1822.902 & 1822.95 & 29 & SQYLDDIATLTGGTVIR & & \\
\hline 1841.839 & 1841.76 & 40 & EPEAAVAGNPMDNSGYGY & & C-term \\
\hline 1900.026 & 1900.04 & 7 & LSGGVAVIQVGAQTETELK & & \\
\hline 2194.234 & 2194.19 & 20 & LSSVANISSTSFGSKRNVALR & & M \\
\hline 2205.213 & 2205.14 & 33 & VAQIKNLIEAADQDYEKEK & & M \\
\hline 2329.543 & 2329.29 & 109 & NVVLESKYGAPKIVNDGVTVAR & & M \\
\hline 2430.329 & 2430.27 & 23 & TNDLAGDGTTTSVVLAQGLIAEGVK & & \\
\hline
\end{tabular}

TC119088, Coverage: $37.2 \%(223$ / 599)

MASTFAGMSSAGPLAAPSTSSNKLSSVANISSTSFGSKRNVALRKSRRPTILAAAKELHFNKDGSAIKKLQNGVNKLADLVGV TLGPKGRNVVLESKYGAPKIVNDGVTVAREVELEDPVENIGAKLVRQAAAKTNDLAGDGTTTSVVLAQGLIAEGVKVVAAGAN PVLITRGIEKTAKALVAELKNMSKEVEDSELADVAAVSAGNNLEVGSMIAEAMSKVGRKGVVTLEEGKSAENSLRVVEGMQFD RGYVSPYFVTDSEKMSVEYENCKLLLVDKKITNARDLVNVLEDAIRNGYPILI IAEDIEQEALATLVVNKLRGALKVAALKAP GFGERKSQYLDDIATLTGGTVIREELGLTLDKADKEVLGHAAKVVLTKDATTIVGDGSTQEAVNKRVAQIKNLIEAADQDYEK EKLNERIAKLSGGVAVIQVGAQTETELKEKKLRVEDALNATKAAVEEGIVVGGGCTLLRLAAKVDAIKGTLANDEEKVGADIV KRALSYPLKLIAKNAGVNGSVVSEKVLSSDDPKFGYNAATGNYEDLMAAG I I DPTKVVRCCLEHAASVAKTFLMSDCVVVEIK EPEAAVAGNPMDNSGYGY

Mr SDS PAGE : $\quad 50 \mathrm{kDa}$ Identified protein(s) : Respiratory burst oxidase protein F (AB064343)

\begin{tabular}{llllll}
\hline exp & cal & ppm & seq & protein & notes \\
\hline 1156.684 & 1156.60 & 72 & THFARPNWK & &
\end{tabular}




\begin{tabular}{llll}
1284.723 & 1284.70 & 21 & THFARPNWKK \\
1360.676 & 1360.65 & 17 & GVMNEVAELDQR \\
1421.746 & 1421.68 & 43 & TTNAYFYWVTR \\
1448.715 & 1448.77 & 41 & QFSQELKAEAVAK \\
1706.825 & 1706.90 & 42 & DSKELALELFDALSR \\
1757.892 & 1758.02 & 73 & KIYPLLLIDGPYGAPK \\
1777.989 & 1777.79 & 111 & MSSDFVYIMQENWK \\
1791.895 & 1791.87 & 12 & LSYCLPLDDNINFHK \\
2262.102 & 2262.29 & 85 & IWVLSLWIMIMIGLFLWK \\
2263.130 & 2263.23 & 43 & VAIYPGNVLTLQMSKPTQFR \\
3338.810 & 3338.68 & 40 & GVIEMHNYLTSVYEEGDARSALITMVQALK \\
\hline
\end{tabular}

\begin{abstract}
AB064343, Coverage: 18.4\% (174/944)
MKPFSKNDRRRWSFDSVSAGKTADGSASTSPGTEYS INGDQEFVEVTIDLQDDDTIVLRSVEPATAINVIGDISDDNTGIMTP VSISRSPTMKRTSSNRFRQFSQELKAEAVAKRKQLSQELKRFSWSRSFSGNLTTTTTDANQRGLGGGGLVNSALEARTLQKSM AQLDRTRSSAQRALRGLRFNSYKQECVDGWNHVQLNFEKFEKNGYIYRSEFAQS IG I KDSKELALELFDALSRRRRLKVEKIN HDELFEYSSQINDESFDSRLQIFFDIVDKNEDGRITEEEVKEIIMLSASANKLSRLKEQAEEYAALIMEELDPERLGYIELWQ LETLLLQKDTYLNCSQALSCTSQALSQNLQGLRGKSRI HRMSSDFVYIMQENWKR IWVLSLWIMIMIGLFLWKFFQYKQKGAF HVMGYCLLTAKGAAGTLQFYMALI PF PVCRNT ITWLRS TRLSYCLPLDDNINFHKTIAGAIVVAVILHIGDHLACDFPRIVRA TEYDYNRYLFHYFQTKQPTYFDLVKGPEGITGILMVILLLFRFTLATRWFRRNLVKLPKPFDRLTGFNAFWYSHHLFVIDYIL LILHGIFLYFAKPWYVRTTWMYLAVPVLRDGGERTLRYFRSGSYSVRLLKVAIYPGNVLTLQMSKPTQFRYKSGQYMFVQCPA VSPFEWHPFS ITSAPEDDY IS I H IRQLGDWTQELKRVFSEVCEPPVGGKSGLLRPAERRKKIYPLLLIDGPYGAPKGDCRLYD VLLLVGLGIGATPFIS I LKDLLNNIVKMEEHADS I S DFSRSSEYSRGSNGHTPRRKRILKTTNAYFYWVTREQGSFDWFKGVM NEVAELDQRGVIEMHNYLTSVYEEGDARSALITMVQALKHAKNGVDIVSGSRVRTHFARPNWKKEVTKLSSKHCNARTGVFFC EVPVLGKELSKLCNTFNQKGSSKFEFHKEHF
\end{abstract}

Mr SDS PAGE : $\quad 30 \mathrm{kDa}$ Identified protein(s) : Unknown (TC120533)

\begin{tabular}{|c|c|c|c|c|c|}
\hline $\exp$ & cal & ppm & seq & protein & notes \\
\hline 1779.838 & 1779.81 & 15 & FIFSSSPSHSSGSPCPR & & \\
\hline 1858.800 & 1859.02 & 119 & AKFLLQNGFGVYNIFK & & \\
\hline 1977.929 & 1978.06 & 66 & SGQATSFLAATTAIVSSPLR & & \\
\hline 1387.646 & 1387.72 & 52 & AAGQEIGTGTVLDR & & \\
\hline 2104.022 & 2104.09 & 32 & FLTTGVSGLAAVGFGVYCVSR & & \\
\hline
\end{tabular}

TC120533, Coverage: $32.5 \%(88 / 271)$ MSESSRWTMPEMAGILSNKI PQDELKPGDH IYSWRNAYLYAHHG I YVGDGMVI HFTRAAGQEIGTGTVLDRFIFSSSPSHSSG SPCPRCGDQSRTEGVISSCLECFLCGGELYLFKYGVSPGVFIAKTRGGTCTIAPSDP PEHVLHRAKFLLQNGFGVYNIFKNNC EDFAIYCKTGLLVFTNVSVGRSGQATSFAATTAIVSSPLRFLTTGVSGLAAVGFGVYCVSRYVSDIGVRRDVMKI PVERLIL CPGPLESDEAATITDTSKEKGS

\title{
Fraction III
}

Mr SDS PAGE : $\quad 150 \mathrm{kDa}$ Identified protein(s) : Alpha-1,4 glucan phosphorylase L-1 (L-1, TC119041) Alpha glucan water dikinase (GWD, TC126433)

\begin{tabular}{llllll}
\hline exp & cal & ppm & seq & protein & notes \\
\hline 912.488 & 912.48 & 5 & ADYFLVGK & L-1 & GWD \\
916.503 & 916.51 & 7 & GITLDELR & L-1 \\
927.494 & 927.42 & 76 & YGSMDLNK & GWD
\end{tabular}




\begin{tabular}{|c|c|c|c|c|c|}
\hline 998.575 & 998.53 & 44 & AFATYVQAK & L-1 & \\
\hline 1015.560 & 1015.59 & 29 & QTILSNIAR & GWD & \\
\hline 1080.548 & 1080.54 & 11 & YWIGGEDIK & L-1 & \\
\hline 1082.579 & 1082.61 & 28 & VLEEPPALSK & GWD & \\
\hline 1082.579 & 1082.48 & 91 & MKEMTAAER & L-1 & $\mathrm{O}, \mathrm{M}$ \\
\hline 1097.577 & 1097.57 & 3 & AFFATAQSVR & L-1 & \\
\hline 1133.581 & 1133.56 & 20 & ADGKFVPDER & L-1 & \\
\hline 1182.614 & 1182.62 & 1 & FEEVKEFVR & L-1 & \\
\hline 1205.641 & 1205.62 & 17 & IKWEEFPEK & L-1 & \\
\hline 1205.641 & 1205.63 & 5 & DLGHYMKTLK & GWD & \\
\hline 1229.459 & 1229.58 & 101 & SDFDLGVYWK & GWD & \\
\hline 1234.611 & 1234.61 & 0 & WTGTEDWVLK & L-1 & \\
\hline 1234.611 & 1234.65 & 32 & IYVVQTRPQM & GWD & C-term \\
\hline 1246.703 & 1246.67 & 23 & AGSAASLSSLLNR & GWD & \\
\hline 1254.737 & 1254.75 & 12 & IRDEILVIQR & GWD & \\
\hline 1258.685 & 1258.66 & 17 & VLSDDINQGVAK & GWD & \\
\hline 1289.664 & 1289.68 & 16 & GLGETLVGAYPGR & GWD & \\
\hline 1292.685 & 1292.69 & 3 & AVAYDVPIPGYK & L-1 & \\
\hline 1331.707 & 1331.76 & 38 & TPVSPEPAVIPPK & L-1 & \\
\hline 1335.734 & 1335.78 & 33 & QLLNIFGIVYR & L-1 & \\
\hline 1382.650 & 1382.73 & 56 & SQSKEEPLHVTK & GWD & \\
\hline 1393.682 & 1393.70 & 10 & LSEGDFSALGEIR & GWD & \\
\hline 1430.744 & 1430.73 & 9 & NDVSYPIKFYGK & L-1 & \\
\hline 1437.756 & 1437.77 & 8 & TNFVPRVCIFGGK & L-1 & \\
\hline 1455.919 & 1455.84 & 53 & TTVLDLSAPAQLVK & GWD & \\
\hline 1459.819 & 1459.85 & 23 & KTPVSPEPAVIPPK & L-1 & \\
\hline 1459.819 & 1459.85 & 23 & TPVSPEPAVIPPKK & L-1 & \\
\hline 1498.729 & 1498.74 & 7 & AIHSEPNFRGDQK & GWD & \\
\hline 1521.750 & 1521.79 & 27 & KLSEGDFSALGEIR & GWD & \\
\hline 1536.631 & 1536.67 & 26 & FADNEDLQNEWR & L-1 & \\
\hline 1579.574 & 1579.75 & 114 & YHAEFTPVFSPER & L-1 & \\
\hline 1591.894 & 1591.93 & 21 & EELRPLLLKPNNR & GWD & \\
\hline 1601.703 & 1601.78 & 49 & GLNWNEAWNITQR & L-1 & \\
\hline 1605.676 & 1605.77 & 60 & QAYYLSMEFLQGR & L-1 & \\
\hline 1615.734 & 1615.77 & 23 & ETWSLPNDRPDGTK & GWD & \\
\hline 1655.768 & 1655.78 & 5 & AAETPFSASSSDGLTSK & GWD & \\
\hline 1674.855 & 1674.84 & 6 & SNIPDDLAQAQAYIR & GWD & \\
\hline 1785.862 & 1785.94 & 42 & TVAYTNHTVLPEALEK & L-1 & \\
\hline 1903.918 & 1903.97 & 25 & LTDLLQNAFTSHPQYR & GWD & \\
\hline 1910.874 & 1910.95 & 39 & VVIDYSSDPLITDGNFR & GWD & \\
\hline 1925.878 & 1925.98 & 53 & TGYSVVPDAMFDIQVKR & L-1 & \\
\hline 1947.925 & 1947.96 & 16 & FGKETWSLPNDRPDGTK & GWD & \\
\hline 2029.044 & 2029.06 & 8 & GPDVSVPEELVQIQSYLR & GWD & \\
\hline 2029.044 & 2029.13 & 44 & ALLNAIGNLELTGAFAEALK & L-1 & $\mathrm{V}$ \\
\hline 2029.044 & 2029.05 & 3 & YGSMDLNKLEEKLTTMR & L-1 & M \\
\hline 2140.067 & 2140.07 & 0 & AGHAIEELYGSPQDIEGVVR & GWD & \\
\hline 2168.109 & 2168.07 & 20 & VAVQMNDTHPTLCIPELMR & L-1 & \\
\hline 2168.109 & 2168.12 & 6 & FITDVGATINHDPEIGDLLK & L-1 & $\mathrm{V}$ \\
\hline 2186.251 & 2186.22 & 13 & DLNSPQVLGYPSKPIGLFIK & GWD & \\
\hline 2194.064 & 2194.10 & 15 & YHAEFTPVFSPERFELPK & L-1 & \\
\hline 2228.229 & 2228.22 & 5 & HVEIIEAIDEELVHEIVLK & L-1 & \\
\hline 2354.185 & 2354.21 & 9 & YWIGGEDIKAVAYDVPIPGYK & L-1 & \\
\hline 2440.588 & 2440.21 & 155 & AGHAIEELYGSPQDIEGVVRDGK & GWD & \\
\hline
\end{tabular}




\begin{tabular}{lllll}
2503.510 & 2503.23 & 112 & NLGHNLENVASQEPDAALGNGGLGR & L-1 \\
2525.250 & 2525.12 & 51 & SGAFGSYNYDDLIGSLEGNEGFGR & L-1 \\
2995.550 & 2995.46 & 29 & IHHPITEQGGESDLSSFAPDAASITSSIK & L-1 \\
\hline
\end{tabular}

TC119041, Coverage: 42.2\% (408/966)

MATANGAHLFNHYSSNSRFIHFTSRNTSSKLFLTKTSHFRRPKRCFHVNNTLSEKIHHPITEQGGESDLSSFAPDAASITSSI KYHAEFTPVFSPERFELPKAFFATAQSVRDSLLINWNATYDIYEKLNMKQAYYLSMEFLQGRALLNAIGNLELTGAFAEALKN LGHNLENVASQEPDAALGNGGLGRLASCFLDSLATLNYPAWGYGLRYKYGLFKQRITKDGQEEVAEDWLEIGSPWEVVRNDVS YPIKFYGKVSTGSDGKRYWIGGEDIKAVAYDVPIPGYKTRTTISLRLWSTQVPSADFDLSAFNAGEHTKACEAQANAEKICYI LYPGDESEEGK I LRLKQQYTLCSASLQDI I SRFERRSGDRIKWEEFPEKVAVQMNDTHPTLCI PELMRILIDLKGLNWNEAWN ITQRTVAYTNHTVLPEALEKWS YELMQKLLPRHVEI IEA IDEELVHEIVLKYGSMDLNKLEEKLTTMR I LENFDLP S SVAELF IKPEISVDDDTETVEVHDKVEASDKVVTNDEDDTGKKTSVKIEAAAEKDIDKKTPVSPEPAVIPPKKVRMANLCVVGGHAVNG VAEIHSEIVKEEVFNDFYELWPEKFQNKTNGVTPRRWIRFCNPPLSAI ITKWTGTEDWVLKTEKLAELQKFADNEDLQNEWRE AKRSNKIKVVSFLKEKTGYSVVPDAMFDIQVKRI HEYKRQLLNIFGIVYRYKKMKEMTAAERKTNEVPRVCIFGGKAFATYVQ AKRIVKFITDVGATINHDPEIGDLLKVVFVPDYNVSVAELLI PASDLSEH I STAGMEASGTSNMKFAMNGCIQIGTLDGANVE IREEVGEENFFLFGAQAHEIAGLRKERADGKFVPDERFEEVKEFVRSGAFGSYNYDDLIGSLEGNEGFGRADYFLVGKDFPSY IECQEKVDEAYRDQKRWTTMS ILNTAGSYKFSSDRTIHEYAKDIWNIEAVEIA

TC126433, Coverage: $22.0 \%(322 / 1464)$

MSNSLGNNLLYQGFLTSTVLEHKSRISP PCVGGNSLFQQQVISKSPLSTEFRGNRLKVQKKKI PMEKNRAFSSSPHAVLTTDT SSQLAEKFSLEGNIELQVDVRPPTSGDVSFVDFQVTNGSDKLFLHWGAVKFGKETWSLPNDRPDGTKVYKNKALRTPFVKSGS NSILRLEIRDTAIEAIEFLIYDEAHDKWIKNNGGNFHIKLSRKEIRGPDVSVPEELVQIQSYLRWERKGKQNYTPEKEKEEYE AARTELQEE IARGAS IQDIRARLTKTNDKSQSKEEPLHVTKSNIPDDLAQAQAY IRWEKAGKPNY P PEKQ IEELEEARRELQL ELEKGITLDELRKKITKGEIKTKVEKHLKRSSFAVERIQRKKRDFGHLINKYTSSPAVQVQKVLEEPPALSKIKLYAKEKEEQ I DDP I LNKKI FKVDDGELLVLVAKSSGKTKVHLATDLNQP I TLHWALSKS PGEWMVPP S S ILPPGS I ILDKAAETPFSASSSD GLTSKVQSLDIVIEDGNFVGMPFVLLSGEKWIKNQGSDFYVGFSAASKLALKAAGDGSGTAKSLLDKIADMESEAQKSFMHRF NIAADLIEDATSAGELGFAGI LVWMRFMATRQL IWNKNYNVKPREISKAQDRLTDLLQNAFTSHPQYREILRMIMSTVGRGGE GDVGQRIRDEILVIQRKNDCKGGMMEEWHQKLHNNTS PDDVVICQALIDYIKSDFDLGVYWKTLNENGITKERLLSYDRAIHS EPNFRGDQKGGLLRDLGHYMKTLKAVHSGADLESAIANCMGYKTEGEGFMVGVQINPVSGLPSGFQDLLHFVLDHVEDKNVET LLERLLEAREELRPLLLKPNNRLKDLLFLDIALDSTVRTAVERGYEELNNANPEKIMYFISLVLENLALSVDDNEDLVYCLKG WNQALSMSNGGDNHWALFAKAVLDRTRLALASKAEWYHHLLQPSAEYLGS ILGVDQWALNIFTEEI IRAGSAASLSSLLNRLD PVLRKTANLGSWQI ISPVEAVGYVVVVDELLSVQNEIYEKPTILVAKSVKGEEEIPDGAVALITPDMP DVLSHVSVRARNGKV CFATCFDPNILADLQAKEGR I LLLKPTPSDI IYSEVNE IELQSSSNLVEAETSATLRLVKKQFGGCYAISADEFTSEMVGAKS RNIAYLKGKVPSSVGI PTSVALPFGVFEKVLSDDINQGVAKELQI LTKKLSEGDFSALGEIRTTVLDLSAPAQLVKELKEKMQ GSGMPWPGDEGPKRWEQAWMA I KKVWASKWNERAYFSTRKVKLDHDYLCMAVLVQE I INADYAFVIHTTNPSSGDDSEIYAEV VRGLGETLVGAYPGRALSFICKKKDLNSPQVLGYPSKPIGLFIKRSI IFRSDSNGEDLEGYAGAGLYDSVPMDEEEKVVIDYS SDPLITDGNFRQTILSNIARAGHAIEELYGSPQDIEGVVRDGKIYVVQTRPQM

Mr SDS PAGE : $\quad 140 \mathrm{kDa}$

Identified protein(s) : Alpha-1,4 glucan phosphorylase L-1 (L-1, TC119041)

Alpha glucan water dikinase (GWD, TC126433)

\begin{tabular}{llllll}
\hline exp & cal & ppm & seq & protein & notes \\
\hline 912.484 & 912.48 & 2 & ADYFLVGK & L-1 & \\
927.496 & 927.42 & 78 & YGSMDLNK & L-1 & \\
998.547 & 998.53 & 17 & AFATYVQAK & L-1 & \\
1080.525 & 1080.54 & 10 & YWIGGEDIK & L-1 & \\
1082.521 & 1082.61 & 81 & VLEEPPALSK & GWD & \\
1082.521 & 1082.48 & 38 & MKEMTAAER & L-1 & O, M \\
1097.562 & 1097.57 & 11 & AFFATAQSVR & L-1 & \\
1100.555 & 1100.57 & 17 & AGKPNYPPEK & GWD & \\
1100.555 & 1100.55 & 0 & WSYELMQK & L-1 & O, M \\
1175.610 & 1175.60 & 12 & VWASKWNER & GWD & \\
1182.585 & 1182.62 & 25 & FEEVKEFVR & L-1 & L-1 \\
1205.630 & 1205.62 & 8 & IKWEEFPEK &
\end{tabular}




\begin{tabular}{|c|c|c|c|c|c|}
\hline 1205.630 & 1205.63 & 4 & DLGHYMKTLK & GWD & \\
\hline 1234.580 & 1234.61 & 24 & WTGTEDWVLK & L-1 & \\
\hline 1234.580 & 1234.66 & 66 & IYVVQTRPQM & GWD & C-term \\
\hline 1246.716 & 1246.67 & 33 & AGSAASLSSLLNR & GWD & \\
\hline 1254.725 & 1254.75 & 22 & IRDEILVIQR & GWD & \\
\hline 1289.634 & 1289.68 & 40 & GLGETLVGAYPGR & GWD & \\
\hline 1292.667 & 1292.69 & 17 & AVAYDVPIPGYK & L-1 & \\
\hline 1318.768 & 1318.67 & 72 & RWEQAWMAIK & GWD & \\
\hline 1331.708 & 1331.76 & 37 & TPVSPEPAVIPPK & L-1 & \\
\hline 1335.765 & 1335.78 & 10 & QLLNIFGIVYR & L-1 & \\
\hline 1393.667 & 1393.70 & 20 & LSEGDFSALGEIR & GWD & \\
\hline 1394.671 & 1394.70 & 17 & FVPDERFEEVK & L-1 & \\
\hline 1430.719 & 1430.73 & 8 & NDVSYPIKFYGK & L-1 & \\
\hline 1455.863 & 1455.84 & 15 & TTVLDLSAPAQLVK & GWD & \\
\hline 1459.819 & 1459.85 & 23 & KTPVSPEPAVIPPK & L-1 & \\
\hline 1459.819 & 1459.85 & 23 & TPVSPEPAVIPPKK & L-1 & \\
\hline 1498.718 & 1498.74 & 14 & AIHSEPNFRGDQK & GWD & \\
\hline 1521.770 & 1521.79 & 14 & KLSEGDFSALGEIR & GWD & \\
\hline 1536.642 & 1536.67 & 19 & FADNEDLQNEWR & L-1 & \\
\hline 1577.679 & 1577.72 & 28 & NQGSDFYVGFSAASK & GWD & \\
\hline 1579.696 & 1579.75 & 37 & YHAEFTPVFSPER & L-1 & \\
\hline 1591.886 & 1591.93 & 26 & EELRPLLLKPNNR & GWD & \\
\hline 1602.085 & 1601.78 & 189 & GLNWNEAWNITQR & L-1 & \\
\hline 1605.691 & 1605.77 & 51 & QAYYLSMEFLQGR & L-1 & \\
\hline 1605.691 & 1605.68 & 7 & MQGSGMPWPGDEGPK & GWD & $2 \mathrm{O}, \mathrm{M}$ \\
\hline 1615.790 & 1615.77 & 12 & ETWSLPNDRPDGTK & GWD & \\
\hline 1655.712 & 1655.78 & 39 & AAETPFSASSSDGLTSK & GWD & \\
\hline 1785.863 & 1785.94 & 42 & TVAYTNHTVLPEALEK & $\mathrm{L}-1$ & V \\
\hline 1785.863 & 1785.85 & 7 & TGYSVVPDAMFDIQVK & L-1 & $\mathrm{O}, \mathrm{M}$ \\
\hline 1903.891 & 1903.97 & 39 & LTDLLQNAFTSHPQYR & GWD & \\
\hline 1910.881 & 1910.95 & 36 & VVIDYSSDPLITDGNFR & GWD & \\
\hline 1925.929 & 1925.98 & 26 & TGYSVVPDAMFDIQVKR & L-1 & $\mathrm{V}$ \\
\hline 1925.929 & 1925.92 & 5 & FVPDERFEEVKEFVR & L-1 & M \\
\hline 2029.231 & 2029.06 & 84 & GPDVSVPEELVQIQSYLR & GWD & \\
\hline 2029.231 & 2029.13 & 49 & ALLNAIGNLELTGAFAEALK & L-1 & \\
\hline 2140.020 & 2140.07 & 22 & AGHAIEELYGSPQDIEGVVR & GWD & \\
\hline 2168.118 & 2168.12 & 2 & FITDVGATINHDPEIGDLLK & L-1 & \\
\hline 2168.118 & 2168.07 & 24 & VAVQMNDTHPTLCIPELMR & L-1 & $\mathrm{V}$ \\
\hline 2186.195 & 2186.22 & 13 & DLNSPQVLGYPSKPIGLFIK & GWD & \\
\hline 2194.094 & 2194.10 & 1 & YHAEFTPVFSPERFELPK & L-1 & \\
\hline 2194.094 & 2194.08 & 6 & EKEEYEAARTELQEEIAR & GWD & M \\
\hline 2440.290 & 2440.21 & 33 & AGHAIEELYGSPQDIEGVVRDGK & GWD & \\
\hline 2503.244 & 2503.23 & -6 & NLGHNLENVASQEPDAALGNGGLGR & L-1 & \\
\hline 2525.205 & 2525.12 & 33 & SGAFGSYNYDDLIGSLEGNEGFGR & L-1 & \\
\hline 2995.579 & 2995.46 & 38 & IHHPITEQGGESDLSSFAPDAASITSSIK & L-1 & \\
\hline
\end{tabular}

TC119041, Coverage: $37.6 \%(371 / 966)$

MATANGAHLFNHYSSNSRFIHFTSRNTSSKLFLTKTSHFRRPKRCFHVNNTLSEKIHHPITEQGGESDLSSFAPDAASITSSI KYHAEFTPVFSPERFELPKAFFATAQSVRDSLL INWNATYD IYEKLNMKQAYYLSMEFLQGRALLNAIGNLELTGAFAEALKN LGHNLENVASQEPDAALGNGGLGRLASCELDSLATLNYPAWGYGLRYKYGLFKQRITKDGQEEVAEDWLEIGSPWEVVRNDVS YPIKFYGKVSTGSDGKRYWIGGEDIKAVAYDVPIPGYKTRTTISLRLWSTQVPSADFDLSAFNAGEHTKACEAQANAEKICYI LYPGDESEEGK I LRLKQQYTLCSASLQDI I SRFERRSGDRIKWEEFPEKVAVQMNDTHPTLCIPELMRI LI DLKGLNWNEAWN ITQRTVAYTNHTVLPEALEKWSYELMQKLLPRHVE I IEA I DEELVHE IVLKYGSMDLNKLEEKLTTMRI LENEDLPSSVAELF 
IKPEISVDDDTETVEVHDKVEASDKVVTNDEDDTGKKTSVKIEAAAEKDI DKKTPVSPEPAVI PPKKVRMANLCVVGGHAVNG VAEIHSE IVKEEVFNDFYELWPEKFQNKTNGVTPRRWIRFCNPP LSA I ITKWTGTEDWVLKTEKLAELQKFADNEDLQNEWRE AKRSNK I KVVSFLKEKTGYSVVPDAMFDIQVKR I HEYKRQLLNIFGIVYRYKKMKEMTAAERKTNFVPRVC IFGGKAFATYVQ AKRIVKFITDVGATINHDPEIGDLLKVVFVPDYNVSVAELLI PASDLSEH I STAGMEASGTSNMKFAMNGC IQI GTLDGANVE IREEVGEENFFLFGAQAHEIAGLRKERADGKFVPDERFEEVKEFVRSGAFGSYNYDDLIGSLEGNEGFGRADYFLVGKDFPSY IECQEKVDEAYRDQKRWTTMS ILNTAGSYKESSDRTIHEYAKDIWNIEAVEIA

TC126433, Coverage: $22.0 \%$ (322/ 1464)

MSNSLGNNLLYQGFLTSTVLEHKSRIS P PCVGGNSLFQQQVISKS PLSTEFRGNRLKVQKKKI PMEKNRAFSSSPHAVLTTDT SSQLAEKFSLEGNIELQVDVRPPTSGDVSFVDFQVTNGS DKLFLHWGAVKFGKETWSLPNDRPDGTKVYKNKALRTPFVKSGS NS I LRLE IRDTA IEA IEF L IYDEAHDKWI KNNGGNFHIKLSRKE IRGPDVSVPEELVQIQSYLRWERKGKQNYTPEKEKEEYE AARTELQEEIARGAS IQDIRARLTKTNDKSQSKEEPLHVTKSNI P DDLAQAQAY IRWEKAGKPNYPPEKQ IEELEEARRELQL ELEKGITLDELRKKITKGE IKTKVEKHLKRSSFAVERIQRKKRDFGHL INKYTSSPAVQVQKVLEEPPALSKIKLYAKEKEEQ I DDP I LNKK I FKVDDGELLVLVAKSSGKTKVHLATDLNQP I TLHWALSKS P GEWMVP PSS I LP PGS I I LDKAAETPFSASSSD GLTSKVQSLDIVIEDGNFVGMPFVLLSGEKWIKNQGSDFYVGFSAASKLALKAAGDGSGTAKSLLDKIADMESEAQKS FMHRF NIAADL IEDATSA GELGFA GI LVWMRFMATRQL IWNKNYNVKPRE I SKAQDRLTDLLQNAFTSHPQYRE I LRMIMSTVGRGGE GDVGQRIRDEILVIQRKNDCKGGMMEEWHQKLHNNT SPDDVVICQALI DY I KS DFDLGVYWKT LNENG ITKERLLSYDRAIHS EPNFRGDQKGGLLRDLGHYMKTLKAVHSGADLESAIANCMGYKTEGEGFMVGVQINPVSGLPSGFQDLLHFVLDHVEDKNVET LLERLLEAREELRPLLLKPNNRLKDLLFLDIALDSTVRTAVERGYEELNNANPEKIMYFISLVLENLALSVDDNEDLVYCLKG WNQALSMSNGGDNHWALFAKAVLDRTRLALASKAEWYHHLLQPSAEY LGS I LGVDQWALNIFTEEI IRAGSAASLSSLLNRLD PVLRKTANLGSWQI I S PVEAVGYVVVVDELLSVQNE IYEKPT I LVAKSVKGEEE I PDGAVALIT P DMP DVLSHVSVRARNGKV CFATCFDPNI LADLQAKEGR I LLLKPTPSDI IYSEVNE IELQSSSNLVEAETSATLRLVKKQFGGCYAI SADEFTSEMVGAKS RNIAYLKGKVPSSVGI PTSVALPFGVFEKVLSDDINQGVAKELQI LTKKLSEGDFSALGEIRTTVLDLSAPAQLVKELKEKMQ GSGMPWPGDEGPKRWEQAWMAIKKVWASKWNERAYFSTRKVKLDHDYLCMAVLVQE I INADYAFV I HTTNPSSGDDSE IYAEV VRGLGETLVGAYPGRALSF ICKKKDLNSPQVLGYPSKPIGLFIKRS I I FRS DSNGEDLEGYAGAGLYDSVPMDEEEKVVIDYS SDPLITDGNFRQTILSNIARAGHAIEELYGSPQDIEGVVRDGKIYVVQTRPQM

Mr SDS PAGE : $\quad 110 \mathrm{kDa}$

Identified protein(s) : Alpha-1,4 glucan phosphorylase L-1 (TC119041)

\begin{tabular}{|c|c|c|c|c|c|}
\hline $\exp$ & cal & ppm & seq & protein & notes \\
\hline 912.477 & 912.48 & 6 & ADYFLVGK & & \\
\hline 1080.558 & 1080.54 & 20 & YWIGGEDIK & & \\
\hline 1084.516 & 1084.51 & 3 & WSYELMQK & & \\
\hline 1097.618 & 1097.57 & 41 & AFFATAQSVR & & \\
\hline 1123.439 & 1123.53 & 81 & VDEAYRDQK & & \\
\hline 1182.602 & 1182.62 & 12 & FEEVKEFVR & & \\
\hline 1205.666 & 1205.62 & 38 & IKWEEFPEK & & \\
\hline 1234.668 & 1234.61 & 46 & WTGTEDWVLK & & \\
\hline 1272.575 & 1272.65 & 57 & DIWNIEAVEIA & & C-term \\
\hline 1292.748 & 1292.69 & 46 & AVAYDVPIPGYK & & \\
\hline 1331.743 & 1331.76 & 10 & TPVSPEPAVIPPK & & \\
\hline 1335.865 & 1335.78 & 65 & QLLNIFGIVYR & & \\
\hline 1394.690 & 1394.70 & 4 & FVPDERFEEVK & & \\
\hline 1430.691 & 1430.73 & 28 & NDVSYPIKFYGK & & \\
\hline 1459.834 & 1459.85 & 12 & KTPVSPEPAVIPPK & & \\
\hline 1459.834 & 1459.85 & 12 & TPVSPEPAVIPPKK & & \\
\hline 1536.712 & 1536.67 & 26 & FADNEDLQNEWR & & \\
\hline 1572.766 & 1572.77 & 5 & WTTMSILNTAGSYK & & \\
\hline 1579.793 & 1579.75 & 25 & YHAEFTPVFSPER & & V \\
\hline 1579.793 & 1579.76 & 21 & WSYELMQKLLPR & & $\mathrm{O}, \mathrm{M}$ \\
\hline 1601.820 & 1601.78 & 24 & GLNWNEAWNITQR & & \\
\hline 1649.751 & 1649.84 & 54 & EMTAAERKTNFVPR & & M \\
\hline 1715.729 & 1715.78 & 31 & ICYILYPGDESEEGK & & \\
\hline 1769.835 & 1769.88 & 24 & TGYSVVPDAMFDIQVK & & \\
\hline
\end{tabular}




\begin{tabular}{lllll}
1785.939 & 1785.94 & 0 & TVAYTNHTVLPEALEK & \\
1844.881 & 1844.84 & 24 & EEVFNDFYELWPEK & \\
1926.003 & 1925.98 & 12 & TGYSVVPDAMFDIQVKR & M \\
1958.013 & 1957.95 & 30 & DSLLINWNATYDIYEK & \\
2029.175 & 2029.13 & 21 & ALLNAIGNLELTGAFAEALK & \\
2168.178 & 2168.12 & 25 & FITDVGATINHDPEIGDLLK & $\mathrm{V}$ \\
2168.178 & 2168.07 & 51 & VAVQMNDTHPTLCIPELMR & \\
2194.120 & 2194.10 & 11 & YHAEFTPVFSPERFELPK & \\
2228.292 & 2228.22 & 34 & HVEIIEAIDEELVHEIVLK & $\mathrm{O}$ \\
2443.239 & 2443.14 & 40 & DGQEEVAEDWLEIGSPWEVVR \\
2463.235 & 2463.19 & 17 & EEVGEENFFLFGAQAHEIAGLR & \\
2463.235 & 2463.23 & 6 & MANLCVVGGHAVNGVAEIHSEIVK & \\
2503.361 & 2503.23 & 53 & NLGHNLENVASQEPDAALGNGGLGR \\
2525.294 & 2525.12 & 68 & SGAFGSYNYDDLIGSLEGNEGFGR & \\
2591.397 & 2591.29 & 42 & EEVGEENFFLFGAQAHEIAGLRK & $\mathrm{V}$ \\
2785.474 & 2785.37 & 38 & ITKDGQEEVAEDWLEIGSPWEVVR & \\
2995.698 & 2995.46 & 78 & IHHPITEQGGESDLSSFAPDAASITSSIK & \\
\hline
\end{tabular}

TC119041, Coverage: $53.7 \%(519 / 966)$

MATANGAHLFNHYSSNSRF IHFTSRNTSSKLFLTKTSHFRRPKRCFHVNNTLSEKIHHPITEQGGESDLSSFAPDAASITSSI KYHAEFTPVFS PERFELPKAFFATAQSVRDSLLINWNATYDIYEKLNMKQAYYLSMEFLQGRALLNAIGNLELTGAFAEALKN LGHNLENVASQEPDAALGNGGLGRLASCFLDSLATLNYPAWGYGLRYKYGLFKQRITKDGQEEVAEDWLEIGSPWEVVRNDVS YPIKFYGKVSTGSDGKRYWIGGEDIKAVAYDVPIPGYKTRTTISLRLWSTQVPSADFDLSAFNAGEHTKACEAQANAEKICYI LYPGDESEEGK I LRLKQQYTLCSASLQDI I SRFERRSGDRIKWEEFPEKVAVQMNDTHPTLCI PELMRILIDLKGLNWNEAWN ITQRTVAYTNHTVLPEALEKWSYELMOKLLPRHVE I IEA IDEELVHEIVLKYGSMDLNKLEEKLTTMRILENFDLPSSVAELF IKPEISVDDDTETVEVHDKVEASDKVVTNDEDDTGKKTSVKIEAAAEKDI DKKTPVSPEPAVIPPKKVRMANLCVVGGHAVNG VAEIHSEIVKEEVFNDFYELWPEKFQNKTNGVT PRRW IRFCNP PLSA I ITKWTGTEDWVLKTEKLAELQKFADNEDLQNEWRE AKRSNKIKVVSFLKEKTGYSVVPDAMFDIQVKRI HEYKRQLLNIFGIVYRYKKMKEMTAAERKTNFVPRVCIFGGKAFATYVQ AKRIVKFITDVGATINHDPEIGDLLKVVFVPDYNVSVAELLIPASDLSEH ISTAGMEASGTSNMKEAMNGCIQIGTLDGANVE IREEVGEENFFLFGAQAHEIAGLRKERADGKFVPDERFEEVKEFVRSGAFGSYNYDDLIGSLEGNEGFGRADYFLVGKDFPSY IECQEKVDEAYRDQKRWTTMS ILNTAGSYKF SS DRT I HEYAKD IWNIEAVEIA

Mr SDS PAGE : $\quad 100 \mathrm{kDa}$

Identified protein(s) : 9-13 Lipoxygenase (9-13 LOX, TC112107)

Lipoxygenase (LOX, TC112595)

Alpha-1,4 glucan phosphorylase L-1 (TC119041)

\begin{tabular}{llllll}
\hline exp & cal & ppm & seq & protein & notes \\
\hline 858.458 & 858.47 & 17 & LQEFPPK & $9-13$ LOX, LOX & \\
899.505 & 899.53 & 34 & LPQGPLFK & $9-13$ LOX, LOX & 9-13 LOX, LOX \\
914.630 & 914.57 & 64 & FPTPLVIK & $9-13$ LOX & Unique \\
919.483 & 919.52 & 45 & IFDVLGQK & $9-13$ LOX, LOX & \\
945.635 & 945.53 & 111 & LLYPHFR & L-1 \\
1080.603 & 1080.54 & 62 & YWIGGEDIK & L-1 \\
1097.633 & 1097.57 & 54 & AFFATAQSVR & $9-13$ LOX, LOX & Unique \\
1139.823 & 1139.58 & 213 & ELREVGHGDK & L-1 & $9-13$ LOX \\
1154.669 & 1154.63 & 33 & AFATYVQAKR & $9-13$ LOX, LOX & \\
1161.588 & 1161.59 & 2 & DKEPLAAFDR & $9-13$ LOX, LOX & L-1 \\
1171.681 & 1171.69 & 12 & QLSVLHPIHK & L-1 & Unique, M \\
1189.711 & 1189.56 & 124 & DNELQAWWK & 9-13 LOX &
\end{tabular}




\begin{tabular}{|c|c|c|c|c|c|}
\hline 1244.719 & 1244.72 & 5 & FPTPLVIKDSK & 9-13 LOX, LOX & \\
\hline 1292.727 & 1292.65 & 63 & NMHINEFFLK & 9-13 LOX & Unique \\
\hline 1292.727 & 1292.72 & 5 & AVAYDVPIPGYK & L-1 & M \\
\hline 1298.787 & 1298.75 & 28 & ALTAAIPLEMIR & 9-13 LOX, LOX & \\
\hline 1306.688 & 1306.71 & 8 & YRENELLTLR & 9-13 LOX, LOX & \\
\hline 1331.820 & 1331.76 & 47 & TPVSPEPAVIPPK & L-1 & \\
\hline 1332.669 & 1332.65 & 11 & HTTDEIYLGQR & 9-13 LOX, LOX & \\
\hline 1336.170 & 1335.78 & 94 & QLLNIFGIVYR & L-1 & \\
\hline 1371.732 & 1371.76 & 19 & ELLRTDGEGILR & 9-13 LOX, LOX & \\
\hline 1381.644 & 1381.65 & 4 & TAWRTDEEFAR & 9-13 LOX, LOX & \\
\hline 1411.767 & 1411.69 & 58 & TTLGGSAEYPYPR & 9-13 LOX, LOX & \\
\hline 1434.763 & 1434.81 & 32 & KYRENELLTLR & 9-13 LOX, LOX & \\
\hline 1452.872 & 1452.86 & 8 & IFFVNQPYLPSK & LOX & Unique \\
\hline 1459.823 & 1459.85 & 19 & KTPVSPEPAVIPPK & L-1 & \\
\hline 1459.823 & 1459.85 & 19 & TPVSPEPAVIPPKK & L-1 & \\
\hline 1536.733 & 1536.67 & 40 & FADNEDLQNEWR & L-1 & \\
\hline 1579.894 & 1579.75 & 88 & YHAEFTPVFSPER & L-1 & $\mathrm{V}$ \\
\hline 1579.894 & 1579.82 & 47 & WSYELMQKLLPR & L-1 & $\mathrm{O}, \mathrm{M}$ \\
\hline 1601.786 & 1601.78 & 3 & GLNWNEAWNITQR & L-1 & \\
\hline 1621.739 & 1621.76 & 13 & QAYYLSMEFLQGR & L-1 & $\mathrm{O}, \mathrm{M}$ \\
\hline 1659.983 & 1659.95 & 22 & LYEGGIKLPQGPLFK & 9-13 LOX, LOX & \\
\hline 1713.817 & 1713.88 & 40 & DWVFPDQALPADLVK & 9-13 LOX, LOX & \\
\hline 1755.963 & 1756.00 & 21 & TDGEGILRFPTPLVIK & 9-13 LOX, LOX & \\
\hline 1785.885 & 1785.94 & 30 & TVAYTNHTVLPEALEK & L-1 & \\
\hline 1890.016 & 1890.03 & 9 & QILVNAGGVLESTVFQSK & 9-13 LOX & Unique \\
\hline 1925.959 & 1925.98 & 10 & TGYSVVPDAMFDIQVKR & L-1 & \\
\hline 2007.981 & 2008.00 & 10 & VYTPSDQGVESSIWQLAK & 9-13 LOX, LOX & \\
\hline 2019.004 & 2019.02 & 9 & LLYPHFRDTMNINASAR & 9-13 LOX & Unique \\
\hline 2019.004 & 2019.17 & 80 & LFILNHHDVLIPYLRR & LOX & Unique \\
\hline 2075.091 & 2075.08 & 7 & VSFQLISSVQGDPTNGLQGK & 9-13 LOX, LOX & \\
\hline 2135.129 & 2135.12 & 6 & IFFANQPYLPSETPELLR & 9-13 LOX & Unique \\
\hline 2168.132 & 2168.07 & 30 & VAVQMNDTHPTLCIPELMR & L-1 & $\mathrm{V}$ \\
\hline 2168.132 & 2168.12 & 4 & FITDVGATINHDPEIGDLLK & L-1 & \\
\hline 2206.184 & 2206.14 & 20 & SGPVNAPYTLLFPTSEGGLTGK & 9-13 LOX, LOX & \\
\hline 2228.163 & 2228.22 & 25 & HVEIIEAIDEELVHEIVLK & L-1 & \\
\hline 2263.180 & 2263.21 & 14 & IFFANQPYLPSETPELLRK & 9-13 LOX & Unique \\
\hline 2263.180 & 2263.17 & 4 & TDEEFAREMLAGVNPVIISR & LOX & $\mathrm{O}$, Unique, $\mathrm{M}$ \\
\hline 2277.237 & 2277.23 & 3 & TDEEFAREMLAGVNPIIISR & 9-13 LOX & $\mathrm{O}$, Unique \\
\hline 2308.200 & 2308.21 & 5 & LLIEDYPYAVDGLEIWSAIK & 9-13 LOX, LOX & \\
\hline 2345.188 & 2345.09 & 42 & LDPEAYGNQNSTITAEHIEDK & 9-13 LOX, LOX & \\
\hline 2501.815 & 2501.16 & 262 & IYDYDIYNDLGNPDEGKENVR & LOX & Unique, $\mathrm{M}$ \\
\hline 2525.205 & 2525.12 & 33 & SGAFGSYNYDDLIGSLEGNEGFGR & L-1 & \\
\hline
\end{tabular}

TC112107, Coverage: $42.9 \%$ (369/861)

MIGQITSGLFGGHDDSKKVKGTVVMMNKNVLDFTDLASSLTGKIFDVLGQKVSFQLISSVQGDPTNGLQGKHSNPAYLENSLF TLTPLTAGSETAFGVTFDWNEEFGVPGA I IKNMHINEFFLKS LTLEDVPNHGKVHFVCNSWVYPS LNYKSDRIFFANQPYLP SETPELLRKYRENELLTLRGDGTGKREAWDRIYDYDIYNDLGNPDQGKENVRTTLGGSAEYPYPRRGRTGRPPTRTDPKSESR IPLILSLDIYVPRDERFGHLKMSDFLTYALKS IVQF ILPELHALFDGTPNEFDSFEDVLRLYEGGIKLPQGPLFKALTAAIPL EMIRELLRTDGEGILRFPTPLVIKDSKTAWRTDEEFAREMLAGVNPIIISRLQEFPPKSKLDPEAYGNQNSTITAEHIEDKLD GLTVDEAMNNNKLFILNHHDV I I PYLRRINTTITKTYASRTLLFLQDNGSLKPLAIELSLPHPDGDQFGVTSKVYTPSDQGVE SSIWQLAKAYVAVNDTGVHQL I S HWLNTHAVIEPFVIATNRQLSVLHPIHKLLYPHFRDTMNINASARQILVNAGGVLESTVF QSKFAMEMSAVVYKDWVFPDQALPADLVKRGVAVEDSSSPHGVRLLIEDYPYAVDGLEIWSAIKSWVTDYCSFYYGSDEEILK DNELQAWWKELREVGHGDKKNE PWWPEMKTPQEL I DSCTT I IWIASALHAAVNFGQYPYAGYLPNRPTVSRRFMPEPGTPEYE 
ELKRNPDKAFLKTITAQLQTLLGVSLVEILSRHTTDEIYLGQRESPEWTKDKEPLAAFDRFGKKLTDIEKQI IQRNGDNILTN RSGPVNAPYTLLFPTSEGGLTGKGI PNSVS I

TC112595, Coverage: 35\% (301/860)

MIGQIKSGLFGPPDDSKKLKGTVVMMNKNALGFTDLAGSLTDTAFDVLGQKVSFQLISSVQGDPTNGLQGKHSNPAYLENSLF TLPPLTVDETSFGVTFDWNEEFGVPGAFI I KNTH INEFFLKSLTLEDVPNHGKVHFVCNSWVYPSFSYKSDRIFFVNQPYLPS KTPELLRKYRENELLTLRGDGTGKREAWDRIYDYDIYNDLGNPDEGKENVRTTLGGSAEYPYPRRGRTGRPPTRTDPKSESRI

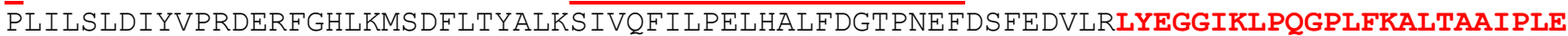
MIRELLRTDGEGILRFPTPLVIKDSKTAWRTDEEFAREMLAGVNPVIISRLQEFPPKSKLDPEAYGNQNSTITAEHIEDKLDG LTVDEAMNNNKLFILNHHDVLIPYLRRINTTTTKTYASRTLLFLQDNGSLKPLAIELSLPHPDGDQFGVTSKVYTPSDQGVES SIWQLAKAYVAVNDSGVHQLISHWLNTHAVIEPFVIATNRQLSVLHPIHKLLYPHFRDTMN INAMARQIL INAGGVLESTVFP SKFAMEMSAVVYKDWVF'PDQALPADLVKRGVAVEDS S SP HGVRLLIEDYPYAVDGLEIWSAIKSWVTDYCSFYYGSDEEILKD NELQAWWKELREVGHGDKKNEPWWPEMERPQEL I DSCTTI IWIASALHAAVNFGQYPYAGYLPNRPTVSRRFMPEPGTPEYEE LKKNPDKAFLKTITAQLQTLLGVSLIEILSRHTTDEIYLGQRESPEWTKDKEPLAAFDKFGKKLTDIEKQI IQRNGDNILTNR SGPVNAPYTLLFPTSEGGLTGKGIPNSVSI

TC119041, Coverage: $27.3 \%(264 / 966)$

MATANGAHLFNHYSSNSRFIHFTSRNTSSKLFLTKTSHFRRPKRCEHVNNTLSEKIHHPITEQGGESDLSSFAPDAASITSS I KYHAEFTPVFSPERFELPKAFFATAQSVRDSLLINWNATYDIYEKLNMKQAYYLSMEFLQGRALLNA IGNLELTGAFAEALKN LGHNLENVASQEPDAALGNGGLGRLASCELDSLATLNYPAWGYGLRYKYGLFKQRITKDGQEEVAEDWLEIGSPWEVVRNDVS YPIKFYGKVSTGSDGKRYWIGGEDIKAVAYDVPIPGYKTRTTISLRLWSTQVPSADFDLSAFNAGEHTKACEAQANAEKICYI LYPGDESEEGKILRLKQQYTLCSASLQDI ISRFERRSGDRIKWEEFPEKVAVQMNDTHPTLCIPELMRILIDLKGLNWNEAWN ITQRTVAYTNHTVLPEALEKWSYELMQKLLPRHVEI IEAIDEELVHEIVLKYGSMDLNKLEEKLTTMRI LENFDLPSSVAELF IKPEISVDDDTETVEVHDKVEASDKVVTNDEDDTGKKTSVKIEAAAEKDIDKKTPVSPEPAVIPPKKVRMANLCVVGGHAVNG VAE I HSEIVKEEVFNDFYELWPEKFQNKTNGVTPRRWIRFCNPPLSAI ITKWTGTEDWVLKTEKLAELQKFADNEDLQNEWRE AKRSNKIKVVSFLKEKTGYSVVPDAMF'DIQVKRI HEYKRQLLNIFGIVYRYKKMKEMTAAERKTNFVPRVCIFGGKAFATYVQ AKRIVKFITDVGATINHDPEIGDLLKVVFVPDYNVSVAELLI PASDLSEH ISTAGMEASGTSNMKFAMNGCIQIGTLDGANVE IREEVGEENFFLFGAQAHEIAGLRKERADGKFVPDERFEEVKEFVRSGAFGSYNYDDLIGSLEGNEGFGRADYFLVGKDFPSY IECQEKVDEAYRDQKRWTTMS I LNTAGSYKFSSDRTIHEYAKDIWNIEAVEIA

Mr SDS PAGE : $\quad 75 \mathrm{kDa}$

Identified protein(s) : Heat shock protein Hsp90-2-like (TC118998)

\begin{tabular}{|c|c|c|c|c|c|}
\hline $\exp$ & cal & ppm & seq & protein & notes \\
\hline 891.403 & 891.42 & 24 & FYEAFSK & & \\
\hline 963.503 & 963.56 & 62 & SKFAELLR & & \\
\hline 1020.462 & 1020.47 & 5 & EEYAAFYK & & \\
\hline 1053.490 & 1053.53 & 34 & APFDLFDTK & & \\
\hline 1133.568 & 1133.58 & 14 & AVENSPFLEK & & \\
\hline 1209.579 & 1209.63 & 39 & RAPFDLFDTK & & \\
\hline 1234.545 & 1234.60 & 46 & DTSGENLGRGTK & & \\
\hline 1256.684 & 1256.70 & 10 & ADLVNNLGTIAR & & \\
\hline 1261.625 & 1261.68 & 42 & KAVENSPFLEK & & \\
\hline 1304.619 & 1304.65 & 22 & EVSNEWSLVNK & & \\
\hline 1320.615 & 1320.66 & 32 & HFSVEGQLEFK & & \\
\hline 1323.643 & 1323.61 & 28 & EDQLEYLEER & & \\
\hline 1527.757 & 1527.80 & 29 & GIVDSEDLPLNISR & & \\
\hline 1541.727 & 1541.76 & 21 & SLTNDWEEHLAVK & & \\
\hline 1560.804 & 1560.82 & 12 & ELISNSSDALDKIR & & \\
\hline 1845.856 & 1845.93 & 38 & KPEEITKEEYAAFYK & & \\
\hline 2426.177 & 2426.06 & 47 & HNDDEQYVWESQAGGSFTVTR & & \\
\hline
\end{tabular}

TC118998, Coverage: $19.2 \%(169 / 881)$

MSDIETFAFQAE INQLLSLIINTFYSNKEIFLRELISNSSDALDKIRFESLTDKSKLDAQPELFIHI IPDKTNNTLTI IDSGI GMTKADLVNNLGTIARSGTKEFMEAIAAGADVSMI GQFGVGFYSAYLVAEKVIVTTKHNDDEQYVWESQAGGSFTVTRDTSGE 
NLGRGTKMVLYLKEDQLEYLEERRLKDLIKKHSEF I SYP I SLWVEKT IEKE I SDDEEEEEKKDEEGKVEEVDEDKEKEEKKKK KVKEVSNEWSLVNKQKP IWMRKPEEITKEEYAAFYKSLTNDWEEHLAVKHFSVEGQLEFKAVLFVPKRAPFDLFDTKKKPNNI KLYVRRVFIMDNCEELI PEYLSFVKGIVDSEDLPLNISRETLQQNKILKVIRKNLVKKCVELFFEIAENKEDYNKFYEAFSKN LKLGI HEDSQNRSKFAELLRYHSTKSGDEMTSLKDYVTRMKEGQNDIYYITGESKKAVENSPFLEKLKKKGYEVLYMVDAIDE YSIGQLKEFEGKKLVSATKEGLKLDESEDEKKKQEELKEKFEGLCKVMKDVLGDKVEKVIVSDRVVDSPCCLVTGEYGWTANM ERIMKAQALRDSSMAGYMSSKKTMEINPENSIMDELRKRADADKNDKSVKDLVLLLFETALLTSGFSLEEPNTFGNRIHRMLK LGLS I DEESGDVDADMPALEDPEADAEGSKMEEVIKFI INVLIVYGFPSYLFS SFCF I P SEQYVIGFNA ILLEWQFHVGYNSL FVLTEVVNI ISFSCPKKNKCHFVAIDIFTGKKLEDIVPSSHNCDVPHVNRTDYVIDISEDGFVSLLTDNGNTKDDLRLPTDDS LLSQIKDGFAEGKDLVVSVMSAMGEEQINGLKDIGPSNLLMGLLDAKFLY

Mr SDS PAGE : $\quad 70 \mathrm{kDa}$

Identified protein(s) : Heat shock protein Hsp90-2-like (TC118998)

\begin{tabular}{llllll}
\hline exp & cal & ppm & seq & protein & notes \\
\hline 891.410 & 891.42 & 16 & FYEAFSK & \\
1133.587 & 1133.58 & 3 & AVENSPFLEK & \\
1209.630 & 1209.63 & 3 & RAPFDLFDTK & \\
1234.576 & 1234.60 & 21 & DTSGENLGRGTK & \\
1256.655 & 1256.70 & 32 & ADLVNNLGTIAR & \\
1304.656 & 1304.65 & 6 & EVSNEWSLVNK & \\
1320.674 & 1320.66 & 12 & HFSVEGQLEFK & \\
1527.790 & 1527.80 & 8 & GIVDSEDLPLNISR \\
1541.800 & 1541.76 & 26 & SLTNDWEEHLAVK \\
1601.896 & 1601.75 & 93 & SGDEMTSLKDYVTR & \\
1617.241 & 1616.74 & 307 & EGQNDIYYITGESK & \\
1678.811 & 1678.87 & 34 & TNNTLTIIDSGIGMTK & \\
1834.826 & 1834.94 & 61 & HSEFISYPISLWVEK & \\
\hline
\end{tabular}

TC118998, Coverage: $18.0 \%(159 / 881)$

MSDIETFAFQAEINQLLSLIINTFYSNKEIFLRELISNSSDALDKIRFESLTDKSKLDAQPELFIHIIPDKTNNTLTIIDSGI GMTKADLVNNLGTIARSGTKEFMEAIAAGADVSMIGQFGVGFYSAYLVAEKVIVTTKHNDDEQYVWESQAGGSFTVTRDTSGE NLGRGTKMVLYLKEDQLEYLEERRLKDLIKKHSEFISYPISLWVEKTIEKEISDDEEEEEKKDEEGKVEEVDEDKEKEEKKKK KVKEVSNEWSLVNKQKP I WMRKPEEITKEEYAAFYKSLTNDWEEHLAVKHFSVEGQLEFKAVLFVPKRAPFDLFDTKKKPNNI KLYVRRVFIMDNCEELI PEYLSFVKGIVDSEDLPLNISRETLQQNKI LKVIRKNLVKKCVELFFEIAENKEDYNKFYEAFSKN LKLGI HEDSQNRSKFAELLRYHSTKSGDEMTSLKDYVTRMKEGQNDIYYITGESKKAVENSPFLEKLKKKGYEVLYMVDAIDE YSIGQLKEFEGKKLVSATKEGLKLDESEDEKKKQEELKEKFEGLCKVMKDVLGDKVEKVIVSDRVVDSPCCLVTGEYGWTANM ERIMKAQALRDS SMAGYMSSKKTMEINPENS IMDELRKRADADKNDKSVKDLVLLLFETALLTSGFSLEEPNTFGNRIHRMLK LGLS IDEESGDVDADMPALEDPEADAEGSKMEEVD

Mr SDS PAGE : $\quad 45 \mathrm{kDa}$ Identified protein(s) : Patatin2-k1 (Kuras, DQ114417)

\begin{tabular}{llllll}
\hline exp & cal & ppm & seq & protein & notes \\
\hline 628.372 & 628.36 & 19 & WGPLR & & \\
1047.468 & 1047.62 & 144 & TNKPVIFTK & \\
1133.526 & 1133.66 & 115 & YLLQVLQEK & \\
1305.578 & 1305.67 & 69 & LAQEDPAFSSIK & \\
1437.572 & 1437.69 & 79 & DSPETYEEALKR & \\
1757.810 & 1757.94 & 76 & VHQALTEVAISSFDIK & \\
1853.734 & 1853.93 & 107 & QMLLLSLGTGTNSEFDK & \\
2783.640 & 2783.46 & 66 & GIIPATILEFLEGQLQEVDNNKDAR & & \\
\hline
\end{tabular}

DQ114417, Coverage: $28.9 \%$ (105/363) 
TLGEMVTVLS I DGGGIKGIIPATILEFLEGQLQEVDNNKDARLADYFDVIGGTSTGGLLTAMITTPNENNRPFAAAKDIVPFY FEHGPH I FNSSGT IFGPMYDGKYLLQVLQEKLGETRVHQALTEVAISSFDIKTNKPVIFTKSNLAKSPELDAKMYDICYSTAA APIYFPPHYFVTHTSNGDRYEFNLVDGAVATVGDPALLSLSVATRLAQEDPAFSSIKSLDYKQMLLLSLGTGTNSEFDKTYTA EEAAKWGPLRWLLAIQQMTNAASSYMTDYYLSTVFQARHSQNNYLRVQENALTGTTTEMDDASEANMELLVQVGETLLKKPVS KDSPETYEEALKRFAKLLSDRKKLRANKASY

\section{Fraction IV}

Mr SDS PAGE : $\quad 150 \mathrm{kDa}$

Identified protein(s) : Alpha-glucan water dikinase (GWD, TC126433)

\begin{tabular}{|c|c|c|c|c|c|}
\hline $\exp$ & cal & ppm & seq & protein & notes \\
\hline 916.503 & 916.51 & 7 & GITLDELR & & \\
\hline 946.931 & 946.50 & 460 & EISKAQDR & & \\
\hline 1015.560 & 1015.59 & 29 & QTILSNIAR & & V \\
\hline 1015.569 & 1015.55 & 21 & KIPMEKNR & & M \\
\hline 1082.579 & 1082.61 & 28 & VLEEPPALSK & & \\
\hline 1205.641 & 1205.63 & 5 & DLGHYMKTLK & & \\
\hline 1229.459 & 1229.58 & 101 & SDFDLGVYWK & & \\
\hline 1246.703 & 1246.67 & 23 & AGSAASLSSLLNR & & \\
\hline 1254.737 & 1254.75 & 12 & IRDEILVIQR & & \\
\hline 1258.685 & 1258.66 & 17 & VLSDDINQGVAK & & \\
\hline 1289.664 & 1289.68 & 16 & GLGETLVGAYPGR & & \\
\hline 1393.682 & 1393.70 & 10 & LSEGDFSALGEIR & & \\
\hline 1455.919 & 1455.84 & 53 & TTVLDLSAPAQLVK & & \\
\hline 1498.729 & 1498.74 & 7 & AIHSEPNFRGDQK & & \\
\hline 1521.750 & 1521.79 & 27 & KLSEGDFSALGEIR & & \\
\hline 1591.894 & 1591.93 & 21 & EELRPLLLKPNNR & & \\
\hline 1615.734 & 1615.77 & 23 & ETWSLPNDRPDGTK & & \\
\hline 1655.768 & 1655.78 & 5 & AAETPFSASSSDGLTSK & & \\
\hline 1674.855 & 1674.84 & 6 & SNIPDDLAQAQAYIR & & \\
\hline 1903.918 & 1903.97 & 25 & LTDLLQNAFTSHPQYR & & \\
\hline 1910.874 & 1910.95 & 39 & VVIDYSSDPLITDGNFR & & \\
\hline 1947.925 & 1947.96 & 16 & FGKETWSLPNDRPDGTK & & \\
\hline 2029.044 & 2029.06 & 8 & GPDVSVPEELVQIQSYLR & & \\
\hline 2140.067 & 2140.07 & 0 & AIEELYGSPQDIEGVVR & & \\
\hline 2186.251 & 2186.22 & 13 & DLNSPQVLGYPSKPIGLFIK & & \\
\hline 2440.588 & 2440.21 & 155 & AGHAIEELYGSPQDIEGVVRDGK & & \\
\hline
\end{tabular}

TC126433, Coverage: $21.0 \%(308 / 1464)$

MSNSLGNNLLYQGFLTSTVLEHKSRISP PCVGGNSLFQQQVISKSPLSTEFRGNRLKVQKKKIPMEKNRAFSSSPHAVLTTDT SSQLAEKFSLEGNIELQVDVRPPTSGDVSFVDFQVTNGSDKLFLHWGAVKFGKETWSLPNDRPDGTKVYKNKALRTPFVKSGS NSILRLEIRDTAIEA IEFLIYDEAHDKWIKNNGGNFHIKLSRKEIRGPDVSVPEELVQIQSYLRWERKGKQNYTPEKEKEEYE AARTELQEE I ARGAS IQDIRARLTKTNDKSQSKEEPLHVTKSNIPDDLAQAQAYIRWEKAGKPNYP PEKQIEELEEARRELQL ELEKGITLDELRKKITKGEIKTKVEKHLKRSSFAVERIQRKKRDFGHLINKYTSSPAVQVQKVLEEPPALSKIKLYAKEKEEQ I DDP I LNKKIFKVDDGELLVLVAKSSGKTKVHLATDLNQP ITLHWALSKS PGEWMVPPSS ILPPGS I LDKAAETPFSASSSD GLTSKVQSLDIVIEDGNFVGMPFVLLSGEKWIKNQGSDFYVGFSAASKLALKAAGDGSGTAKSLLDKIADMESEAQKSFMHRF NIAADL IEDATSAGELGFAGI LVWMRFMATRQL IWNKNYNVKPREISKAQDRLTDLLQNAFTSHPQYREILRMIMSTVGRGGE GDVGQRIRDEILVIQRKNDCKGGMMEEWHQKLHNNTSPDDVVICQALIDY IKSDFDLGVYWKTLNENGITKERLLSYDRAIHS EPNFRGDQKGGLLRDLGHYMKTLKAVHSGADLESA IANCMGYKTEGEGFMVGVQ INPVSGLPSGFQDLLHFVLDHVEDKNVET LLERLLEAREELRPLLLKPNNRLKDLLFLDIALDSTVRTAVERGYEELNNANPEKIMYFISLVLENLALSVDDNEDLVYCLKG WNQALSMSNGGDNHWALFAKAVLDRTRLALASKAEWYHHLLQPSAEYLGS I LGVDQWALNIFTEE I RAGSAASLSSLLNRLD PVLRKTANLGSWQI I S PVAVGYVVVVDELLSVQNEIYEKPTILVAKSVKGEEEIPDGAVALITPDMPDVLSHVSVRARNGKV CFATCFDPNI LADLQAKEGRILLLKPTPSDI IYSEVNE IELQSSSNLVEAETSATLRLVKKQFGGCYAISADEFTSEMVGAKS 
RNIAYLKGKVPSSVGIPTSVALPFGVFEKVLSDDINQGVAKELQILTKKLSEGDFSALGEIRTTVLDLSAPAQLVKELKEKMQ GSGMPWPGDEGPKRWEQAWMAIKKVWASKWNERAYFSTRKVKLDHDYLCMAVLVQEI INADYAFVIHTTNPSSGDDSEIYAEV VRGLGETLVGAYPGRALSFICKKKDLNSPQVLGYPSKPIGLFIKRS I I FRSDSNGEDLEGYAGAGLYDSVPMDEEEKVVIDYS SDPLITDGNFRQTILSNIARAGHAIEELYGSPQDIEGVVRDGKIYVVQTRPQM

Mr SDS PAGE : $\quad 140 \mathrm{kDa}$

Identified protein(s) : Alpha-1,4 glucan phosphorylase L-1 (TC119041)

\begin{tabular}{|c|c|c|c|c|c|}
\hline $\exp$ & cal & ppm & seq & protein & notes \\
\hline 912.468 & 912.48 & 16 & ADYFLVGK & & \\
\hline 927.474 & 927.42 & 54 & YGSMDLNK & & \\
\hline 998.555 & 998.53 & 24 & AFATYVQAK & & \\
\hline 1080.528 & 1080.54 & 8 & YWIGGEDIK & & \\
\hline 1097.557 & 1097.57 & 15 & AFFATAQSVR & & \\
\hline 1133.561 & 1133.56 & 2 & ADGKFVPDER & & \\
\hline 1182.594 & 1182.62 & 18 & FEEVKEFVR & & \\
\hline 1205.621 & 1205.62 & 1 & IKWEEFPEK & & \\
\hline 1234.591 & 1234.61 & 16 & WTGTEDWVLK & & \\
\hline 1292.665 & 1292.69 & 18 & AVAYDVPIPGYK & & \\
\hline 1331.687 & 1331.76 & 53 & TPVSPEPAVIPPK & & \\
\hline 1335.714 & 1335.78 & 48 & QLLNIFGIVYR & & \\
\hline 1430.724 & 1430.73 & 5 & NDVSYPIKFYGK & & M \\
\hline 1437.736 & 1437.77 & 22 & TNFVPRVCIFGGK & & \\
\hline 1459.799 & 1459.85 & 36 & KTPVSPEPAVIPPK & & \\
\hline 1459.799 & 1459.85 & 36 & TPVSPEPAVIPPKK & & \\
\hline 1579.554 & 1579.75 & 127 & YHAEFTPVFSPER & & \\
\hline 1605.656 & 1605.77 & 73 & QAYYLSMEFLQGR & & \\
\hline 1785.842 & 1785.94 & 54 & TVAYTNHTVLPEALEK & & $\mathrm{V}$ \\
\hline 1785.842 & 1785.83 & 7 & TGYSVVPDAMFDIQVK & & $\mathrm{O}, \mathrm{M}$ \\
\hline 1925.858 & 1925.98 & 63 & TGYSVVPDAMFDIQVKR & & $\mathrm{V}$ \\
\hline 1925.858 & 1925.96 & 53 & FVPDERFEEVKEFVR & & M \\
\hline 2029.024 & 2029.13 & 53 & ALLNAIGNLELTGAFAEALK & & \\
\hline 2168.089 & 2168.07 & 11 & VAVQMNDTHPTLCIPELMR & & \\
\hline 2168.089 & 2168.12 & 16 & FITDVGATINHDPEIGDLLK & & $\mathrm{V}$ \\
\hline 2194.044 & 2194.10 & 24 & YHAEFTPVFSPERFELPK & & \\
\hline 2228.209 & 2228.22 & 4 & HVEIIEAIDEELVHEIVLK & & \\
\hline 2354.165 & 2354.21 & 18 & YWIGGEDIKAVAYDVPIPGYK & & \\
\hline 2525.230 & 2525.12 & 43 & SGAFGSYNYDDLIGSLEGNEGFGR & & \\
\hline 2995.530 & 2995.46 & 22 & IHHPITEQGGESDLSSFAPDAASITSSIK & & \\
\hline
\end{tabular}

TC119041, Coverage: $35.2 \%(340 / 966)$

MATANGAHLFNHYSSNSRF IHFTSRNTSSKLFLTKTSHFRRPKRCFHVNNTLSEKIHHPITEQGGESDLSSFAPDAASITSSI KYHAEFTPVFSPERFELPKAFFATAQSVRDSLL INWNATYDIYEKLNMKQAYYLSMEFLQGRALLNAIGNLELTGAFAEALKN LGHNLENVASQEPDAALGNGGLGRLASCFLDSLATLNYPAWGYGLRYKYGLFKQRITKDGQEEVAEDWLEIGSPWEVVRNDVS YPIKFYGKVSTGSDGKRYWIGGEDIKAVAYDVPIPGYKTRTTISLRLWSTQVPSADFDLSAFNAGEHTKACEAQANAEKICYI LYPGDESEEGKILRLKQQYTLCSASLQDI I SRFERRSGDRIKWEEFPEKVAVQMNDTHPTLCIPELMRILIDLKGLNWNEAWN ITQRTVAYTNHTVLPEALEKWSYELMQKLLPRHVEI IEAIDEELVHEIVLKYGSMDLNKLEEKLTTMRI LENFDLPSSVAELF IKPEISVDDDTETVEVHDKVEASDKVVTNDEDDTGKKTSVKIEAAAEKDIDKKTPVSPEPAVIPPKKVRMANLCVVGGHAVNG VAEIHSEIVKEEVFNDFYELWPEKFQNKTNGVTPRRWIRFCNPPLSAI ITKWTGTEDWVLKTEKLAELQKEADNEDLQNEWRE AKRSNKIKVVSFLKEKTGYSVVPDAMFDIQVKRI HEYKRQLLNIFGIVYRYKKMKEMTAAERKTNFVPRVCIFGGKAFATYVQ AKRIVKFITDVGATINHDPEIGDLLKVVFVPDYNVSVAELLI PASDLSEH ISTAGMEASGTSNMKFAMNGCIQIGTLDGANVE IREEVGEENFFLFGAQAHEIAGLRKERADGKFVPDERFEEVKEFVRSGAFGSYNYDDLIGSLEGNEGFGRADYFLVGKDFPSY IECQEKVDEAYRDQKRWTTMS ILNTAGSYKFSSDRT IHEYAKDIWNIEAVEIA 
Mr SDS PAGE : $\quad 105 \mathrm{kDa}$

Identified protein(s) : Alpha-1,4 glucan phosphorylase L-1 (L-1, TC119041)

1,4-alpha-glucan branching enzyme, Q-enzyme (Q, TC119364)

\begin{tabular}{|c|c|c|c|c|c|}
\hline $\exp$ & cal & ppm & seq & protein & notes \\
\hline 1107.593 & 1107.56 & 31 & DQFGVWSIR & Q & $\mathrm{V}$ \\
\hline 1107.593 & 1107.56 & 28 & VGCDLPGKYR & Q & \\
\hline 1112.720 & 1112.46 & 230 & EGNNWSYDK & Q & \\
\hline 1292.687 & 1292.69 & 1 & AVAYDVPIPGYK & L-1 & \\
\hline 1335.763 & 1335.78 & 11 & QLLNIFGIVYR & L-1 & \\
\hline 1424.742 & 1424.73 & 10 & FIHFTSRNTSSK & L-1 & \\
\hline 1424.742 & 1424.73 & 7 & LFNYANWEVLR & Q & \\
\hline 1437.751 & 1437.77 & 11 & TNFVPRVCIFGGK & L-1 & \\
\hline 1579.814 & 1579.75 & 38 & YHAEFTPVFSPER & L-1 & $\mathrm{V}$ \\
\hline 1579.814 & 1579.82 & 4 & WSYELMQKLLPR & L-1 & $\mathrm{O}, \mathrm{M}$ \\
\hline 1601.799 & 1601.78 & 11 & GLNWNEAWNITQR & L-1 & \\
\hline 1605.853 & 1605.77 & 50 & QAYYLSMEFLQGR & L-1 & \\
\hline 1616.775 & 1616.75 & 18 & VALDSDAWEFGGHGR & Q & \\
\hline 1643.793 & 1643.77 & 12 & NTYEGYKVGCDLPGK & Q & $\mathrm{V}$ \\
\hline 1643.793 & 1643.80 & 1 & YEGPLEEFAQGYLK & Q & \\
\hline 1661.744 & 1661.74 & 3 & WWLEEYNFDGFR & Q & \\
\hline 1673.734 & 1673.90 & 97 & IPDVDSKPVIPHNSR & Q & \\
\hline 1785.879 & 1785.94 & 33 & TVAYTNHTVLPEALEK & L-1 & $\mathrm{V}$ \\
\hline 1785.879 & 1785.86 & 10 & TGYSVVPDAMFDIQVK & L-1 & $\mathrm{O}, \mathrm{M}$ \\
\hline 1864.904 & 1864.85 & 31 & FADNEDLQNEWREAK & L-1 & $\mathrm{V}$ \\
\hline 1957.987 & 1957.95 & 17 & DSLLINWNATYDIYEK & L-1 & \\
\hline 1966.904 & 1966.90 & 4 & FAAPYDGVYWDPPPSER & Q & \\
\hline 2085.019 & 2084.98 & 19 & WIDYLKNKNDEDWSMK & Q & M \\
\hline 2168.135 & 2168.12 & 5 & FITDVGATINHDPEIGDLLK & L-1 & \\
\hline 2168.135 & 2168.07 & 32 & VAVQMNDTHPTLCIPELMR & L-1 & $\mathrm{V}$ \\
\hline 2168.135 & 2168.00 & 62 & NDEDWSMKEVTSSLTNRR & $\mathrm{Q}$ & M \\
\hline 2228.220 & 2228.22 & 1 & HVEIIEAIDEELVHEIVLK & L-1 & \\
\hline 2257.212 & 2257.04 & 74 & YTEKCIAYAESHDQSIVGDK & Q & \\
\hline 2305.186 & 2305.15 & 15 & DSLSTNISNIDQTVVVSVEER & Q & \\
\hline 2443.242 & 2443.14 & 41 & DGQEEVAEDWLEIGSPWEVVR & L-1 & \\
\hline 2463.288 & 2463.19 & 38 & EEVGEENFFLFGAQAHEIAGLR & L-1 & V \\
\hline 2463.288 & 2463.24 & 19 & MANLCVVGGHAVNGVAEIHSEIVK & L-1 & $\mathrm{O}, \mathrm{M}$ \\
\hline 2470.315 & 2470.26 & 22 & TENIGLLNLDPTLEPYLDHFR & Q & \\
\hline 2503.316 & 2503.23 & 35 & NLGHNLENVASQEPDAALGNGGLGR & L-1 & \\
\hline 2525.247 & 2525.12 & 50 & SGAFGSYNYDDLIGSLEGNEGFGR & L-1 & \\
\hline 2995.723 & 2995.46 & 86 & IHHPITEQGGESDLSSFAPDAASITSSIK & L-1 & \\
\hline
\end{tabular}

TC112041, Coverage: $37.8 \%(365 / 966)$

MATANGAHLFNHYSSNSRFIHFTSRNTSSKLFLTKTSHFRRPKRCFHVNNTLSEKIHHPITEQGGESDLSSFAPDAASITSSI KYHAEFTPVFSPERFELPKAFFATAQSVRDSLLINWNATYDIYEKLNMKQAYYLSMEFLQGRALLNAIGNLELTGAFAEALKN LGHNLENVASQEPDAALGNGGLGRLASCFLDSLATLNYPAWGYGLRYKYGLFKQRITKDGQEEVAEDWLEIGSPWEVVRNDVS YPIKFYGKVSTGSDGKRYWIGGEDIKAVAYDVPIPGYKTRTTISLRLWSTQVPSADFDLSAFNAGEHTKACEAQANAEKICYI LYPGDESEEGKILRLKQQYTLCSASLQDI I SRFERRSGDRIKWEEFPEKVAVQMNDTHPTLCIPELMRILIDLKGLNWNEAWN ITQRTVAYTNHTVLPEALEKWSYELMQKLLPRHVEI IEAIDEELVHEIVLKYGSMDLNKLEEKLTTMRI LENEDLP SSVAELF IKPEISVDDDTETVEVHDKVEASDKVVTNDEDDTGKKTSVKIEAAAEKDI DKKTPVSPEPAVI P PKKVRMANLCVVGGHAVNG VAEIHSEIVKEEVFNDFYELWPEKFQNKTNGVTPRRWIRFCNPPLSAI ITKWTGTEDWVLKTEKLAELQKFADNEDLQNEWRE AKRSNKIKVVSFLKEKTGYSVVPDAMFDIQVKRI HEYKRQLLNIFGIVYRYKKMKEMTAAERKTNFVPRVCIFGGKAFATYVQ AKRIVKFITDVGATINHDPEIGDLLKVVFVPDYNVSVAELLI PASDLSEH ISTAGMEASGTSNMKEAMNGCIQIGTLDGANVE 
IREEVGEENFFLFGAQAHEIAGLRKERADGKFVPDERFEEVKEFVRSGAFGSYNYDDLIGSLEGNEGFGRADYFLVGKDFPSY IECQEKVDEAYRDQKRWTTMS I LNTAGSYKFSSDRT IHEYAKDIWNIEAVEIA

TC119364, Coverage: $22.1 \%(207 / 937)$

MLFQLNGMIQMQTSGVRTYKKAAAEAKYHNLINGINFNVLSKPIRGSFPSFS PKVSSGASRNKICFPSQHSTGLKFGSQERSW DISSTPKSRVRKDERMKHSSA ISAVLTDDNSTMAPLEEDVKTENIGLLNLDPTLEPYLDHFRHRMKRYVDQKMLIEKYEGPLE EFAQGYLKFGFNREDGC IVYREWAPAAQEAEVIGDFNGWNGSNHMMEKDQFGVWSIRIPDVDSKPVIPHNSRVKFRFKHGNGV WVDRI PAWIKYATADATKFAAPYDGVYWDPPPSERYHFKYPRPPKPRAPRIYEAHVGMSSSEPRVNSYREFADDVLPRIKANN YNTVQLMAIMEHSYYGSFGYHVTNFFAVSSRYGNPEDLKYLIDKAHSLGLQVLVDVVHSHASNNVTDGLNGFDIGQGSQESYF HAGERGYHKLWDSRLFNYANWEVLRFLLSNLRWWLEEYNFDGFRFDGITSMLYVHHG INMGFTGNYNEYFSEATDVDAVVYLM LANNLI HKI F P DATVIAEDVS GMP GLSRPVSEGG I GFDYRLAMA I PDKWIDYLKNKNDEDWSMKEVTSSLTNRRYTEKCIAYA ESHDQSIVGDKTIAFLLMDKEMYSGMSCLTDASPVVDRGIALHKMIHFFTMALGGEGYLNFMGNEFGHPEW IDFPREGNNWSY DKCRRQWNLADSEHLRYKFMNAFDRAMNSLDEKFSFLASGKQIVSSMDDDNKVVVFERGDLVFVFNFHPKNTYEGYKVGCDLP GKYRVALDSDAWEFGGHGRTGHDVDHFTSPEGI PGVPETNFNGRPNSFKVLSPARTCVAYYRVDERMSETEDYQTDICSELLP TANIEESDEKLKDSLSTNISNI DERMSETEVYQTDISSELLPTANIEESDEKLKDSLSTNISNIDQTVVVSVEERDKELKDSP SVSIISDVVPAEWDDSDANVWGED

Mr SDS PAGE : $\quad 100 \mathrm{kDa}$ Identified protein(s) : Lipoxygenase (TC112595)

\begin{tabular}{|c|c|c|c|c|c|}
\hline $\exp$ & cal & ppm & seq & protein & notes \\
\hline 1188.595 & 1188.60 & 2 & MSDFLTYALK & & \\
\hline 1275.559 & 1275.61 & 41 & FAMEMSAVVYK & & \\
\hline 1298.725 & 1298.75 & 19 & ALTAAIPLEMIR & & \\
\hline 1398.727 & 1398.78 & 36 & EMLAGVNPVIISR & & \\
\hline 1511.869 & 1511.92 & 33 & IPLILSLDIYVPR & & \\
\hline 1713.787 & 1713.88 & 57 & DWVFPDQALPADLVK & & \\
\hline 1862.952 & 1863.06 & 60 & LFILNHHDVLIPYLR & & \\
\hline 2007.915 & 2008.00 & 43 & VYTPSDQGVESSIWQLAK & & \\
\hline 2206.067 & 2206.14 & 33 & SGPVNAPYTLLFPTSEGGLTGK & & \\
\hline 2308.134 & 2308.21 & 33 & LLIEDYPYAVDGLEIWSAIK & & \\
\hline 2354.113 & 2354.19 & 32 & NALGFTDLAGSLTDTAFDVLGQK & & M \\
\hline 2502.055 & 2502.14 & 35 & IYDYDIYNDLGNPDEGKENVR & & \\
\hline
\end{tabular}

TC119595, Coverage: 22.4\% (193/860)

MIGQIKSGLFGPPDDSKKLKGTVVMMNKNALGFTDLAGSLTDTAFDVLGQKVSFQLISSVQGDPTNGLQGKHSNPAYLENSLF TLPPLTVDETSFGVTFDWNEEFGVPGAFI IKNTH INEFFLKSLTLEDVPNHGKVHFVCNSWVYPSFSYKSDRIFFVNQPYLPS KTPELLRKYRENELLTLRGDGTGKREAWDRIYDYDIYNDLGNPDEGKENVRTTLGGSAEYPYPRRGRTGRPPTRTDPKSESRI PLILSLDIYVPRDERFGHLKMSDFLTYALKS IVQFILPELHALFDGTPNEFDSFEDVLRLYEGGIKLPQGPLFKALTAAIPLE MIRELLRTDGEGILRFPTPLVIKDSKTAWRTDEEFAREMLAGVNPVIISRLQEFPPKSKLDPEAYGNQNSTITAEH IEDKLDG LTVDEAMNNNKLFILNHHDVLIPYLRRINTTTTKTYASRTLLFLQDNGSLKPLAIELSLPHPDGDQFGVTSKVYTPSDQGVES SIWQLAKAYVAVNDSGVHQLIS HWLNTHAVIEP FVIATNRQLSVLHP I HKLLYPHFRDTMNINAMARQ I L INAGGVLESTVEP SKFAMEMSAVVYKDWVFPDQALPADLVKRGVAVEDSSSPHGVRLLIEDYPYAVDGLEIWSAIKSWVTDYCSFYYGSDEEILKD NELQAWWKELREVGHGDKKNEPWWPEMERPQELIDSCTTI IWIASALHAAVNFGQYPYAGYLPNRPTVSRREMPEPGTPEYEE LKKNPDKAFLKTITAQLQTLLGVSLIEILSRHTTDE IYLGQRES PEWTKDKEPLAAFDKFGKKLTDIEKQI IQRNGDNI LTNR SGPVNAPYTLLFPTSEGGLTGKGIPNSVS I

Mr SDS PAGE : $\quad 95 \mathrm{kDa}$

Identified protein(s) : Elongation factor EF-2 (EF, TC111732)

Nematode resistance-like protein (TC121585)

\begin{tabular}{|c|c|c|c|c|c|}
\hline $\exp$ & cal & ppm & seq & protein & notes \\
\hline 1107.462 & 1107.57 & 99 & LGVTMKSDEK & $\mathrm{EF}$ & \\
\hline 1107.462 & 1107.53 & 57 & YMNLSHSQK & NM & \\
\hline
\end{tabular}




\begin{tabular}{|c|c|c|c|c|c|}
\hline 1294.558 & 1294.64 & 65 & DSVVAGFQWASK & $\mathrm{EF}$ & $\mathrm{V}$ \\
\hline 1294.558 & 1294.57 & 9 & MTDTRADEAER & $\mathrm{EF}$ & \\
\hline 1360.711 & 1360.70 & 10 & IFLDIACFFSGK & NM & \\
\hline 1360.711 & 1360.58 & 96 & FGVDESKMMER & EF & $2 \mathrm{O}, \mathrm{M}$ \\
\hline 1424.680 & 1424.68 & 3 & LWGENFFDPATK & $\mathrm{EF}$ & \\
\hline 1521.671 & 1521.73 & 37 & SQFQGACFLHEVR & NM & \\
\hline 1605.832 & 1605.85 & 10 & SDPVVSFRETVLEK & $\mathrm{EF}$ & \\
\hline 1634.765 & 1634.84 & 47 & ILAEEFGWDKDLAK & $\mathrm{EF}$ & \\
\hline 1713.818 & 1713.88 & 38 & MNQESSLLPSPEIIR & NM & N-term \\
\hline 1723.858 & 1723.78 & 45 & EATDDPRMCSRLWK & NM & $\mathrm{O}, \mathrm{M}$ \\
\hline 1742.863 & 1742.91 & 28 & AYLPVVESFGFSGTLR & $\mathrm{EF}$ & \\
\hline 1757.886 & 1757.95 & 39 & LIRTPDFSVTPNLER & NM & \\
\hline 1792.894 & 1792.85 & 22 & SQFQGACFLHEVRDR & NM & \\
\hline 1807.887 & 1807.93 & 24 & NATLTNEKEVDAHPIR & $\mathrm{EF}$ & \\
\hline 1807.887 & 1807.89 & 2 & GKFISPELMSSIEESR & NM & M \\
\hline 1809.884 & 1809.91 & 12 & GKFISPELMSSIEESR & NM & $\mathrm{V}$ \\
\hline 1864.871 & 1864.86 & 4 & STGISLYYEMTDESLR & $\mathrm{EF}$ & \\
\hline 1879.856 & 1879.95 & 50 & KGLKEQMTPLSEFEDK & $\mathrm{EF}$ & M \\
\hline 2022.926 & 2022.98 & 25 & VENLYEGPLDDAYANAIR & $\mathrm{EF}$ & \\
\hline 2257.200 & 2257.20 & 1 & STLTDSLVAAAGIIAQEVAGDVR & $\mathrm{EF}$ & \\
\hline 2308.217 & 2308.10 & 50 & NCDPEGPLMLYVSKMIPASDK & $\mathrm{EF}$ & \\
\hline 2318.132 & 2318.23 & 44 & VLLVLDDVDHIDQLNALAGER & NM & \\
\hline 2342.153 & 2342.14 & 5 & YRVENLYEGPLDDAYANAIR & $\mathrm{EF}$ & \\
\hline 2436.247 & 2436.31 & 26 & LHCTHTAIQTIPSSMSLLKNLK & NM & \\
\hline
\end{tabular}

TC111732, Coverage: $25.1 \%(212 / 843)$

MVKFTAEELRRIMDFKHNIRNMSVIAHVDHGKSTLTDSLVAAAGI IAQEVAGDVRMTDTRADEAERGITIKSTGISLYYEMTD ESLRNFKGERNGNEYLINLIDSPGHVDFSSEVTAALRITDGALVVVDCVEGVCVQTETVLRQALGERIRPVLTVNKMDRCFLE LQVDGEEAYQTFQRVIENANVIMATYEDPLLGDVQVYPEKGTVAFSAGLHGWAFTLTNFAKMYASKFGVDESKMMERLWGENF FDPATKKWTTKNSGTASCKRGFVQFCYEP I KQ I INTCMNDQKDKLWPMLQKLGVTMKSDEKDLMGKALMKRVMQTWLPASTAL LEMMIYHLPSPAIAQRYRVENLYEGPLDDAYANAIRNCDPEGPLMLYVSKMIPASDKGRFFAFGRVFAGKVCTGMKVRIMGPN YVPGEKKDLYVKNIQRTVIWMGKRQETVEDVPCGNTVAMVGLDQY ITKNATLTNEKEVDAHPIRAMKFSVSPVVRVAVQCKVA SDLPKLVEGLKRLAKS DPMVVCS IEESGEHI IAGAGELHLE ICLKDLQDDFMGGAE I I KSDPVVSFRETVLEKSTRTVMSKSP NKHNRLYMEARPMEEGLAEAIDDGRI GPRDDPKVRSKILAEEFGWDKDLAKK I WCFGPETTGPNMVVDMCKGVQYLNE IKDSV VAGFQWASKEGALAEENMRGICFEVCDVVLHSDA I HRGGGQVIPTARRVIYASQLTAKPRLLEPVYLVEIQAPEQALGGIYSV LNQKRGHVFEEMQRPGTPLYNI KAYLPVVESFGFSGTLRAATSGQAFPQCVFDHWEMMS SDPLEVGSQANQLVLDIRKRKGLK EQMTPLSEFEDKL

TC121585, Coverage: $12.2 \%(139 / 1135)$

MNQESSLLPSPEIIRWSYDVFLSFRGEDVRKTFVDHLYLALQQKC INTEKDDEKLEKGKFISPELMSSIEESRIALI IFSKNY ANSTWCLDELTKIMECKNVKGQIVVPVFYDVDPSTVRKQKS I FGEAFSKHEARFQEDKVQKWRAALEEAANISGWDLPNTSNG HEARVMEKIAEDIMARLGSQRHASNARNLVGMES HMHQVYKMLG I GSGGVHFLGILGMSGVGKTTLARVIYDNIRSQFQGACF LHEVRDRSAKQGLERLQEILLSE I LVVKKLRINDSFEGANMQKQRLQYKKVLLVLDDVDHIDQLNALAGEREWFGDGSRI I IT TKDKHLLVKYETEKIYRMKTLNNYESLQLFKQHAFKKNRPTKEFEDLSAQVIKHTDGLPLALKVLGSFLYGRGLDEWISEVER LKQI PENEI LKKLEQSFTGLHNTEQKIFLDIACFFSGKKKDSVTRILESFHFCPVIGIKVLMEKCLITILQGRITIHQLIQDM GWHIVRREATDDPRMCSRLWKREDICPVLERNLGTDKIEGMSLHLTNEEEVNFGGKAFMQMTRLRFLKFRNAYVCQGPEFLPD ELRWLDWHGYPSKSLPNSFKGDQLVSLKLKKSRI IQLWKTSKDLGKLKYMNLSHSQKLIRTPDFSVTPNLERLVLEECTSLVE INFS IENLGKLVLLNLKNCRNLKTLPKRIRLEKLEILVLTGCSKLRTEPEIEEKMNCLAELYLGATSLSELPASVENLSGVGV INLSYCKHLESLPSS IFRLKCLKTLDVSGCSKLKNLPDDLGLLVGLEKLHCTHTAIQTIPSSMSLLKNLKHLSLSGCNALSSQ VSSS S HGQKSMGVNFQNLSGLCSLIMLDLSDCNISDGGILSNLGFLPSLEVLILDGNNFSNIPAAS ISRLTRLKSLALHGCGR LESLPELPPS IKGIYANECTSLMS IDQLTKYPMLSDVSFRNCHQLVKNKQHTSMVDSLLKQMLEALYMNVRFGLYVPGMEIPE WFTYKSWGTQSMSVALPTNWFTPFRGFTVCVVLDKRMPFILGPFNIHKVHGLKNMIWLNLKRYDGLRQKISTSFGP I GSEKPG GLGNTLITHVAFGSSWQLEDDLDYYRNNAFQLEFSACDHYQKDMVKGLGVRLVYEN

Mr SDS PAGE : $\quad 70 \mathrm{kDa}$ 
Identified protein(s) : Hsp90 (HSP, TC118924)

Transketolase 1 (TK, TC118997)

\begin{tabular}{llllll}
\hline exp & cal & ppm & seq & protein & notes \\
\hline 1209.622 & 1209.66 & 34 & FGASAPAGKIYK & TK & \\
1209.622 & 1209.63 & 3 & RAPFDLFDTK & HSP & TK \\
1313.640 & 1313.72 & 59 & NLSQQNLNALAK & HSP & \\
1320.653 & 1320.66 & 4 & HFSVEGQLEFK & TK & \\
1390.733 & 1390.76 & 18 & EFGITAEAVIAAAK & TK & \\
1424.680 & 1424.71 & 18 & VSIEAGTTFGWEK & TK & \\
1458.729 & 1458.75 & 13 & SIITGELPADWEK & HSP & TK \\
1527.768 & 1527.80 & 22 & GIVDSEDLPLNISR & HSP & M \\
1589.752 & 1589.78 & 18 & ALPTYTPESPADATR & HSP & \\
1589.752 & 1589.79 & 24 & HEELKEKFEGLCK & HSP & \\
1663.761 & 1663.90 & 84 & FEGLCKVIKDVLGDK & HSP & \\
1664.760 & 1664.85 & 55 & TNNTLTIIDSGVGMTK & HSP & HSP \\
1748.871 & 1748.96 & 50 & LDAQPELFIHIIPDK & HSP & M \\
1808.864 & 1808.79 & 40 & TIEKEISDDEDEEEK & HSP & \\
1834.854 & 1834.94 & 45 & HSEFISYPISLWVEK & HSP & \\
1896.847 & 1896.90 & 28 & EDYDKFYEAFSKNLK & TK & \\
1963.980 & 1964.09 & 54 & SKLDAQPELFIHIIPDK & TK & \\
2038.951 & 2039.07 & 58 & ITLFLKEDQLEYLEER & HSP & \\
2068.968 & 2069.01 & 22 & QNLGWPYEPFHVPEEVK & HSP & TK \\
2163.010 & 2163.03 & 8 & VVSFVSWELFDEQSAEYK & TK & \\
2426.110 & 2426.06 & 19 & HNDDEQYVWESQAGGSFTVTR & & \\
2445.235 & 2445.22 & 6 & RVFIMDNCEELIPEYLSFVK & LIAFYDDNHISIDGDTEIAFTEDVAAR & \\
3011.532 & 3011.43 & 35 & 96 & GGYTISDNSSGNKPDVILIGTGSELEIAVK & \\
3034.266 & 3034.56 & 96 & & & \\
\hline
\end{tabular}

TC118924, Coverage: $27.5 \%$ (192 / 699)

MAEAETFAFQAE INQLLS L I INTFYSNKE I FLRELISNSSDALDKIRFQSLTDKSKLDAQPELFIHI IPDKTNNTLTIIDSGV GMTKADLVNNLGTIARSGTKEFMEAIAAGADVSMIGQFGVGFYSAYLVAERVVVTTKHNDDEQYVWESQAGGSFTVTRDTSGE DLGRGTKITLFLKEDQLEYLEERRLKDLIKKHSEFISYPISLWVEKTIEKEISDDEDEEEKKDEEGKVEEVDEEKEKEEKKKK KIKEVSNEWSLVNKQKP IWMRKPEEITKEEYAAFYKSLTNDWEEHLAVKHFSVEGQLEFKAVLF I PKRAPFDLFDTKKKPNNI KLYVRRVFIMDNCEELIPEYLSFVKGIVDSEDLPLNISREMLQQNKILKVIRKNLVKKCIELFFE IAENKEDYDKFYEAFSKN LKLGI HEDSQNRSKFAELLRYHSTKSGDEMTS LKDYVTRMKEGQND IYYITGESKKAVENSPFLEKLKKKGYEVLYMVDAIDE YSIGQLKEFEGKKLVSATKEGLKLEETEDEKKKHEELKEKFEGLCKVIKDVLGDKVEKVVVSDRVVDSPCCLVTGEYGWTANM ERIMKAQALRDSSMAGYMSSKKTMEINPENAIMDELRKRADADKNDKSVKDLVLLLFETALLTSGFSLDEPNTFGNRIHRMLK LGLSIDEDSGDAEADMPALEDPEADAEGSKMEEVD

TC118997, Coverage: $22.9 \%(171 / 746)$

MASSSSLTLSQAILSRSVSRHGSSSSTNSQLSPSSLSLPTESGLKSTTATSFRRHILPSAAASGRHSAIRASAAVETLEKTDN ALVEKSVNTIRFLAIDAVEKANSGHPGLPMGCAPMGHI LYDE IMRYNPKNPYWFNRDRFVLSAGHGCMLQYALLHLAGYDAVR EEDLKSFRQWGSKTPGHPENFETPGVEVTTGPLGQGIANAVGLALSEKHLAARFNKPDAEIVDHYTYCIVGDGCQMEGISQEA ASLAGHWGLGKLIAFYDDNHISIDGDTEIAFTEDVAARYEALGWHVIWVKNGNTGYDEIRAAIKEAKAVTDKPTLIKVTTTIG FGSPNKSNSYSVHGSALGAKEVEATRQNLGWPYEPFHVPEEVKSHWSRHVPEGAALEAGWNSKEAEYEKKYPEEAADLKSIIT GELPADWEKALPTYTPESPADATRNLSQQNLNALAKVLPGFLGGSADLASSNMTLMKMFGDFQKSTPEERNIRFGVREHGMGA ICNGIALHS PGLI PYCATFFVFTDYMRAA IRISALAESRVIYVMTHDS IGLGEDGPTHQP IEHLAS FRAMPNVLMLRPADGNE TAGAYRVAI LKSKTPS I LALSRQKLPQLAGTS IEGTAKGGYTISDNSSGNKPDVILIGTGSELEIAVKAADELRKEGKAVRVV SFVSWELFDEQSAEYKESVLPAAVTARVSIEAGTTFGWEKIVGSKGKA I GI DRFGASAPAGKIYKEFGITAEAVIAAAKQVS

Mr SDS PAGE : $\quad 62 \mathrm{kDa}$

Identified protein(s) : 4-alpha-glucanotransferase D-enzyme (TC112373) 


\begin{tabular}{|c|c|c|c|c|c|}
\hline $\exp$ & cal & ppm & seq & protein & notes \\
\hline 918.518 & 918.55 & 37 & KQFLLNR & & \\
\hline 918.518 & 918.55 & 37 & QFLLNRK & & V \\
\hline 1021.633 & 1021.57 & 62 & DILATYGRL & & C-term, M \\
\hline 1113.550 & 1113.52 & 26 & ATDLFDEFR & & \\
\hline 1177.663 & 1177.67 & 5 & LRDILATYGR & & \\
\hline 1290.708 & 1290.75 & 33 & LRDILATYGRL & & C-term, M \\
\hline 1295.575 & 1295.65 & 56 & EELPEPLPTDR & & \\
\hline 1388.693 & 1388.65 & 32 & GWWDTLPQEEK & & \\
\hline 1423.719 & 1423.74 & 17 & KEELPEPLPTDR & & \\
\hline 1424.705 & 1424.68 & 15 & GFAGFWAVPSEEK & & \\
\hline 1594.712 & 1594.76 & 30 & IPSSTSFDNLDAEAK & & \\
\hline 1710.100 & 1709.99 & 61 & VGPGKPLFDAILQAVGK & & \\
\hline 1722.824 & 1722.85 & 18 & IPSSTSFDNLDAEAKK & & \\
\hline 1781.835 & 1781.86 & 14 & ATDLFDEFRIDHFR & & \\
\hline 1820.956 & 1821.00 & 24 & VNYSTISEIKDPLITK & & \\
\hline 1877.944 & 1877.96 & 9 & LLSSEGELKDQLENFR & & \\
\hline 2034.124 & 2034.06 & 32 & RLLSSEGELKDQLENFR & & M \\
\hline 2553.340 & 2553.35 & 4 & AGILLHPTSFPGPYGIGDLGPQAFK & & \\
\hline
\end{tabular}

TC112373, Coverage: $27.8 \%(160 / 576)$

MAIHTCFSLIPSSFS STKLPFPKNTTFQSPIPKLSRPTFMFDRKGSFQNGTAAVPAVGEDFPIDYADWLPKRDPNDRRRAGIL LHPTSFPGPYGIGDLGPQAFKFLDWLHLAGCSLWQVLPLVP PGKRGNEDGSPYSGQDANCGNTLLISLEELVDDGLLKKEELP EPLPTDRVNYSTISEIKDPLITKAAKRLLSSEGELKDQLENERRDPNISSWLEDAAYFAA I DNSVNTISWYDWPEPLKNRHLA ALEEVYQSEKDF I DIFIAQQFLFQRQWKKVRDYARSKGIS IMGDMP IYVGYHSADVWANKKQFLLNRKGFPLIVSGVPPDAFS ETGQLWGSPLYDWKAMEKDGFSWWVCRIQRATDLFDEFRIDHFRGFAGFWAVPSEEKIAILGRWKVGPGKPLFDAILQAVGKI NIIAEDLGVITEDVVQLRKSIEAPGMAVLQFAFGSDAENPHLPHNHEQNQVVYTGTHDNDTIRGWWDTLPQEEKSNVLKYLSN IEEEE ISWGL IEGAVSSVARIA I I PMQDVLGLGS DSRMN I PATQFGNWSWRIPSSTSFDNLDAEAKKLRDILATYGRL

Mr SDS PAGE : $\quad 60 \mathrm{kDa}$

Identified protein(s) : 4-alpha-glucanotransferase D-enzyme (TC112373)

\begin{tabular}{|c|c|c|c|c|c|}
\hline $\exp$ & cal & ppm & seq & protein & notes \\
\hline 918.477 & 918.55 & 82 & KQFLLNR & & $\mathrm{V}$ \\
\hline 918.477 & 918.55 & 82 & QFLLNRK & & \\
\hline 1021.650 & 1021.57 & 79 & DILATYGRL & & C-term, M \\
\hline 1113.605 & 1113.52 & 75 & ATDLFDEFR & & \\
\hline 1132.712 & 1132.60 & 99 & NTTFQSPIPK & & \\
\hline 1295.694 & 1295.65 & 36 & EELPEPLPTDR & & \\
\hline 1388.771 & 1388.65 & 88 & GWWDTLPQEEK & & \\
\hline 1423.757 & 1423.74 & 10 & KEELPEPLPTDR & & \\
\hline 1424.761 & 1424.68 & 53 & GFAGFWAVPSEEK & & \\
\hline 1594.788 & 1594.76 & 18 & IPSSTSFDNLDAEAK & & \\
\hline 1722.885 & 1722.85 & 18 & IPSSTSFDNLDAEAKK & & \\
\hline 1781.811 & 1781.86 & 28 & ATDLFDEFRIDHFR & & \\
\hline 1821.026 & 1821.00 & 14 & VNYSTISEIKDPLITK & & \\
\hline 1877.940 & 1877.96 & 11 & LLSSEGELKDQLENFR & & \\
\hline 2034.048 & 2034.06 & 6 & RLLSSEGELKDQLENFR & & M \\
\hline 2553.390 & 2553.35 & 15 & AGILLHPTSFPGPYGIGDLGPQAFK & & \\
\hline
\end{tabular}

TC112373, Coverage: $26.2 \%(151 / 576)$

MAI HTCFSLI ISSSFSTKLLPFKNTTFQSPIPKLSRPTFMFDRKGSFQNGTAAVPAVGEDFPI DYADWLPKRDPNDRRRAGII LHPTSFPGPYGIGDLGPQAFKFLDWLHLAGCSLWQVLPLVPPGKRGNEDGSPYSGQDANCGNTLLISLEELVDDGLLKKEELP 
EPLPTDRVNYSTISEIKDPLITKAAKRLLSSEGELKDQLENFRRDPNISSWLEDAAYFAAI DNSVNTISWYDWPEPLKNRHLA ALEEVYQSEKDFIDIFIAQQFLFQRQWKKVRDYARSKGIS IMGDMP IYVGYHSADVWANKKQFLLNRKGFPLIVSGVPPDAFS ETGQLWGSPLYDWKAMEKDGFSWWVCRIQRATDLFDEFRIDHFRGFAGFWAVPSEEKIAILGRWKVGPGKPLFDA ILQAVGKI NIIAEDLGVITEDVVQLRKSIEAPGMAVLQFAFGSDAENPHLPHNHEQNQVVYTGTHDNDTIRGWWDTLPQEEKSNVLKYLSN IEEEEISWGLIEGAVSSVARIA I I PMQDVLGLGS DSRMN I PATQFGNWSWRIPSSTSFDNLDAEAKKLRDILATYGRL

Mr SDS PAGE : $\quad 58 \mathrm{kDa}$

Identified protein(s) : Blight resistance protein SH20 (TC132622)

\begin{tabular}{|c|c|c|c|c|c|}
\hline $\exp$ & cal & ppm & seq & protein & notes \\
\hline 1021.598 & 1021.55 & 51 & YLNISHFK & & \\
\hline 1173.610 & 1173.71 & 84 & GLTSLTELIVK & & \\
\hline 1315.697 & 1315.59 & 83 & EATSFPEEMFK & & \\
\hline 1417.778 & 1417.70 & 58 & IQWCCALENIPK & & V \\
\hline 1417.778 & 1417.72 & 41 & NDKEAKEANLSAK & & M \\
\hline 1437.679 & 1437.76 & 56 & SSLDVKDLASFQK & & \\
\hline 1646.748 & 1646.95 & 123 & IGSLTCLKTLGQSVVK & & \\
\hline 1785.852 & 1785.99 & 80 & VISLWMAHGFLLSRR & & \\
\hline 2034.047 & 2034.03 & 9 & RYLLVLDDVWNEDQQK & & \\
\hline
\end{tabular}

TC132622, Coverage: $12.5 \%(115 / 920)$

MAEAFIQVLLENITSFIQRELGLLLGFENDFENISSRFSTIQAVLEDAQEKQLKDKAIKNWLQKLNADAYKVDDLLDECNYES ARLKQPRLGRHHPKTIAFRHKIGKRIKEMMEKLDAIAKERKDFHLHEKI IERQVARPETGSVLTEPQVYGRDKEEDEIVKILI NNVSNAQELSVLP ILGMGGLGKTTLAQMVFNDQRVTEHFHPKIWICVSDDFDEKRLIENI IGNIERSSLDVKDLASFQKKLQQ LLNGKRYLLVLDDVWNEDQQKWDNLRVVLKVGASGASVLTTTRLEKVGSVMGTLQPYQLSNLSQDDCWLLFIQRAFRHQEE IS PNLVAIGKEIVKKSGGVPLAAKTLGGLLRFKREKREWEHVRDSEIWNLPQDEMS ILPALRLSYHHLPLALRQCFAYCAVFPKD TKMEKKKVISLWMAHGFLLSRRNLELEDVRNEGWNELYLRSFFQE IEVRYGNTYFKMXDL IHDLAXSLLSANTSSSN IRE INV ESYTHMMMS I GFSEVVSSYSP SLLQKFVSLRVLNLSYSKFEELPSS IGDLVHLRYMDLSNNIE IRSLPKQLCKLQNLQTLDLQ YCTRLCCLPKQTSKLGSLRNLLLHGCHRLTRTPPRIGSLTCLKTLGQSVVKRKKGYQLGELGSLNLYGS IKISHLERVKNDKE AKEANLSAKENLHSLSMKWDDDEHPHRYESEEVEVLEALKPHSNLTCLKISGFRGIRLPDWMNHSVLKN IVLIE I SGCKNCSC LPPFGDLPCLESLELYRGSAEYVEEVDIDVDSGFPTRIRLPSLRKLCICKFDNLKGLLKKEGGEQFPVLEEMEIRYCPIPTLS PNLKALTSLNISDNKEATSFPEEMFKSLANLKYLNISHFKNLKELPTSLAS LNALKSLKIQWCCALENIPKEGVKGLTSLTEL IVKFSKV

Mr SDS PAGE : $\quad 50 \mathrm{kDa}$ Identified protein(s) : Neutral leucine aminopeptidase (TC112200)

\begin{tabular}{|c|c|c|c|c|c|}
\hline $\exp$ & cal & ppm & seq & protein & notes \\
\hline 987.471 & 987.56 & 92 & FQNPLLQK & & \\
\hline 1189.635 & 1189.69 & 50 & QGGAITAALFLK & & \\
\hline 1271.542 & 1271.63 & 67 & GLTFDSGGYNIK & & \\
\hline 1292.740 & 1292.76 & 16 & FKSESKKPTLK & & M \\
\hline 1430.734 & 1430.80 & 46 & FQNPLLQKLDSK & & \\
\hline 1488.717 & 1488.78 & 42 & DGNSKFQNPLLQK & & \\
\hline 1541.788 & 1541.84 & 35 & SLDILGLGTGPEIEK & & \\
\hline 1597.722 & 1597.78 & 37 & DTLGLTHTNQSDAPK & & \\
\hline 1653.800 & 1653.88 & 49 & IALVGLGSSTSSTAAYR & & \\
\hline 1670.340 & 1669.94 & 241 & SLDILGLGTGPEIEKK & & M \\
\hline 1720.818 & 1720.93 & 63 & NATGFGVSTLVEWVLK & & \\
\hline 1815.795 & 1815.86 & 38 & LSGLLSEASFEEDFSGK & & \\
\hline 2009.972 & 2010.01 & 21 & LSSASAITTGAVLGTFEDNR & & \\
\hline 2009.972 & 2010.03 & 27 & VQWMHIDLAGPVWSDKK & & V \\
\hline 2048.993 & 2049.12 & 63 & ELVNAPANVLTPAVLAEEAK & & \\
\hline 2105.219 & 2105.07 & 70 & SAQASNIAIALASTDGLSAESK & & \\
\hline
\end{tabular}


TC112200, Coverage: $36.9 \%(213 / 577)$

MAALRVSSALACSSSSSFHSYPSFFTKFQSSPIWSFS ISVTPLCSRRAKRMAHS IARDTLGLTHTNQSDAPKISFAAKE IDLV EWKGDI LAVGATEKDLARDGNSKFQNPLLQKLDSKLSGLLSEASFEEDFSGKVGQST I LRLPGLGSKRIALVGLGSSTSSTAA YRS LGEAAAAAAKSAQASNIAIALASTDGLSAESKLSSASAITTGAVLGTFEDNRFKSESKKPTLKSLDILGLGTGPEIEKKI KYAADVCAGVI LGRELVNAPANVLTPAVLAEEAKKIASTYSDVFSANI LDVEQCKELKMGSYLAVAAASANPAHF I HLSYKPS SGEIKKKIALVGKGLTFDSGGYNIKTGPGCS IELMKF DMGGAAAVLGAAKALGQ IKPAGVEVHF IVAACENMISGTGMRPGDI ITASNGKTIEVNNTDAEGRLTLADALVYACNQGVEKIVDLATLTGACVVALGPS IAGIFTPS DDLAKEVVAASEVSGEKLWRL PMEDSYWDSMKSGVADMVNTGGRQGGAITAALFLKQFVDEKVQWMHIDLAGPVWSDKKKNATGFGVSTLVEWVLKNSTN

\section{Mr SDS PAGE : $\quad 45 \mathrm{kDa}$}

Identified protein(s) : Patatin 3-k1 (Kuras, DQ114421)

\begin{tabular}{|c|c|c|c|c|c|}
\hline $\exp$ & cal & ppm & seq & protein & notes \\
\hline 1047.619 & 1047.62 & 1 & TNKPVIFTK & & \\
\hline 1151.517 & 1151.61 & 81 & YLMQVLQEK & & \\
\hline 1289.592 & 1289.67 & 60 & LAQEDPAFASIK & & Unique \\
\hline 1448.791 & 1448.72 & 49 & DIIPFYFDHGPK & & Unique, \\
\hline 1614.991 & 1614.82 & 106 & YDGKYLMVLQEK & & \\
\hline 1757.881 & 1757.94 & 34 & VHQALTEVAISSFDIK & & \\
\hline 1848.781 & 1848.90 & 64 & KSVSEDNHETYEVALK & & Unique, $\mathrm{V}$ \\
\hline 1848.781 & 1848.89 & 59 & WGILQWMSPLWEMR & & Unique, $\mathrm{O}, \mathrm{M}$ \\
\hline 1876.771 & 1876.90 & 69 & SVSEDNHETYEVALKR & & Unique \\
\hline 1962.817 & 1963.00 & 93 & IFEPSGFHLVEPKYDGK & & Unique \\
\hline 2223.321 & 2223.10 & 99 & SVSEDNHETYEVALKRFAK & & Unique, $\mathrm{M}$ \\
\hline 2786.671 & 2786.55 & 43 & VHQALTEVAISSFDIKTNKPVIFTK & & $\mathrm{V}$ \\
\hline
\end{tabular}

DQ114421, Coverage: 30.0\% (109/363)

TLGEMVTVLS IDGGGIKGI IPATI LEFLEGQLQEVDNNTDARLADYFDVIGGTGTGGLLTAMITTPNENNRPFAAAKDIIPFY FDHGPKIFEPSGFHLVEPKYDGKYLMQVLQEKLGETRVHQALTEVAISSFDIKTNKPVIFTKSNLAKTPELDAKMYDICYSTA AAPTYFP PHYFATNTSNGDQY DFNLVDGDVAAVDPS LLS I SVATRLAQEDPAFAS IKS LNYKQMLLLSLGTGTNSEFAKNYTA EEAAKWGILQWMS PLWEMRSAASS YMNDYYLSTVFQALDSQNNYLRVQENALTGTATTFDDASVANMILLVQVGENLLKKSVS EDNHETYEVALKRFAKLLSDRKKLRANKASE

Mr SDS PAGE : $\quad 42 \mathrm{kDa}$

Identified protein(s) : Patatin 2-k1 (Kuras, DQ114417)

\begin{tabular}{llllll}
\hline exp & cal & ppm & seq & protein & notes \\
\hline 628.372 & 628.36 & 19 & WGPLR & \\
1047.468 & 1047.62 & 144 & TNKPVIFTK & \\
1133.526 & 1133.66 & 115 & YLLQVLQEK & \\
1272.512 & 1272.68 & 132 & SNLAKSPELDAK & \\
1281.592 & 1281.59 & 2 & DSPETYEEALK & \\
1305.578 & 1305.67 & 69 & LAQEDPAFSSIK & \\
1437.572 & 1437.69 & 79 & DSPETYEEALKR & \\
1757.810 & 1757.94 & 76 & VHQALTEVAISSFDIK & \\
1853.734 & 1853.93 & 107 & QMLLLSLGTGTNSEFDK & \\
\hline
\end{tabular}

DQ114417, Coverage: 25.3\% (92/363)

TLGEMVTVLS I DGGGIKGI I PAT I LEFLEGQLQEVDNNKDARLADYFDVIGGTSTGGLLTAMITTPNENNRPFAAAKDIVPFY FEHGPH I FNSS GT I FGPMY DGKYLLQVLQEKLGETRVHQALTEVAISSFDIKTNKPVIFTKSNLAKSPELDAKMYDICYSTAA 
APIYFPPHYFVTHTSNGDRYEFNLVDGAVATVGDPALLSLSVATRLAQEDPAFSSIKSLDYKQMLLLSLGTGTNSEFDKTYTA EEAAKWGPLRWLLAIQQMTNAASSYMTDYYLSTVFQARHSQNNYLRVQENALTGTTTEMDDASEANMELLVQVGETLLKKPVS KDSPETYEEALKREAKLLSDRKKLRANKASY

Mr SDS PAGE : $\quad 37 \mathrm{kDa}$

Identified protein(s) : Succinyl-CoA ligase alpha subunit (TC126340)

\begin{tabular}{llllll}
\hline exp & cal & ppm & seq & protein & notes \\
\hline 1084.718 & 1084.56 & 149 & MGHAGAIVSGGK & & \\
1122.526 & 1122.62 & 87 & IGVTMLDVFK & \\
1617.592 & 1617.83 & 148 & YFGSPPPPPAVFVDK & \\
2039.400 & 2038.14 & 16 & ASGTQKPVVAFIAGLTAPPGR & & \\
\hline
\end{tabular}

TC126340, Coverage: $17.2 \%(58 / 337)$

MARQATRLISNLSTKLNPSSPTISAS PLWHQYRYFGSPPPPPAVF'VDKNTRVICQGITGKNGTFHTEQAIEYGTKMVGGVTPK KGGTEHLGLPVFNTVAEAKAETKANASVVYVPPPFAAAAIMEAMEAELDLVVCITEGI PQHDMVRVKAALKKQSRTRLIGPNC PGI IKPGECKIGIMPGYIHKPGRIGIVSRSGTLTYEAVFQTTAVGLGQSTCVGIGGDPFNGTNFVDCLERFIADPQTEGIVLI GEIGGTAEEDAAALIKASGTQKPVVAFIAGLTAPPGRRMGHAGAIVSGGKGTAQDKIKALKEAGVTVCESPAKIGVTMLDVFK QRGLA

\section{Fraction V}

Mr SDS PAGE : $\quad 99 \mathrm{kDa}$ Identified protein(s) : 9- 13 Lipoxygenase (TC112107)

\begin{tabular}{|c|c|c|c|c|c|}
\hline $\exp$ & cal & ppm & seq & protein & notes \\
\hline 1161.629 & 1161.59 & 34 & DKEPLAAFDR & & \\
\hline 1189.604 & 1189.56 & 34 & DNELQAWWK & & \\
\hline 1298.789 & 1298.75 & 30 & ALTAAIPLEMIR & & \\
\hline 1306.748 & 1306.71 & 28 & YRENELLTLR & & \\
\hline 1412.767 & 1412.79 & 18 & EMLAGVNPIIISR & & \\
\hline 1511.935 & 1511.92 & 10 & IPLILSLDIYVPR & & \\
\hline 1580.781 & 1580.82 & 23 & NVLDFTDLASSLTGK & & \\
\hline 1658.872 & 1658.88 & 5 & ENELLTLRGDGTGKR & & M \\
\hline 1713.869 & 1713.88 & 9 & DWVFPDQALPADLVK & & \\
\hline 1822.883 & 1822.87 & 8 & RFMPEPGTPEYEELK & & $\mathrm{V}$ \\
\hline 1822.883 & 1822.87 & 8 & FMPEPGTPEYEELKR & & \\
\hline 1863.014 & 1863.06 & 27 & LFILNHHDVIIPYLR & & \\
\hline 1869.892 & 1869.99 & 50 & DWVFPDQALPADLVKR & & \\
\hline 1889.995 & 1890.03 & 20 & QILVNAGGVLESTVFQSK & & \\
\hline 2008.032 & 2008.00 & 15 & VYTPSDQGVESSIWQLAK & & \\
\hline 2097.979 & 2098.21 & 109 & QLSVLHPIHKLLYPHFR & & \\
\hline 2135.122 & 2135.12 & 2 & IFFANQPYLPSETPELLR & & \\
\hline 2206.169 & 2206.14 & 14 & SGPVNAPYTLLFPTSEGGLTGK & & \\
\hline 2308.294 & 2308.21 & 36 & LLIEDYPYAVDGLEIWSAIK & & \\
\hline 2345.202 & 2345.09 & 48 & LDPEAYGNQNSTITAEHIEDK & & \\
\hline
\end{tabular}

TC112107, Coverage: $31.4 \%(270 / 861$

MIGQITSGLFGGHDDSKKVKGTVVMMNKNVLDFTDLASSLTGKIFDVLGQKVSFQLISSVQGDPTNGLQGKHSNPAYLENSLF TLTPLTAGSETAFGVTFDWNEEFGVPGAF I IKNMH INEFFLKSLTLEDVPNHGKVHFVCNSWVYPS LNYKSDR IFFANQPYLP SETPELLRKYRENELLTLRGDGTGKREAWDRIYDYDIYNDLGNPDQGKENVRTTLGGSAEYPYPRRGRTGRPPTRTDPKSESR IPLILSLDIYVPRDERFGHLKMSDFLTYALKS IVQF ILPELHALFDGTPNEFDSFEDVLRLYEGGIKLPQGPLFKALTAAIPL EMIRELLRTDGEGILRFPTPLVIKDSKTAWRTDEEFAREMLAGVNPIIISRLQEFPPKSKLDPEAYGNQNSTITAEHIEDKLD 
GLTVDEAMNNNKLFILNHHDVI I PYLRRINTTITKTYASRTLLFLQDNGSLKPLAIELSLPHPDGDQFGVTSKVYTPSDQGVE SSIWQLAKAYVAVNDTGVHQL I S HWLNTHAVIEP FVIATNRQLSVLHPIHKLLYPHFRDTMNINASARQILVNAGGVLESTVF QSKFAMEMSAVVYKDWVFPDQALPADLVKRGVAVEDSSSPHGVRLLIEDYPYAVDGLEIWSAIKSWVTDYCSFYYGSDEEILK DNELQAWWKELREVGHGDKKNEPWWPEMKTPQELI DSCTT I IWIASALHAAVNFGQYPYAGYLPNRPTVSRRFMPEPGTPEYE ELKRNPDKAFLKT ITAQLQTLLGVSLVEI LSRHTTDEIYLGQRES PEWTKDKEPLAAFDRFGKKLTDIEKQ I IQRNGDNI LTN RSGPVNAPYTLLFPTSEGGLTGKGIPNSVSI

Mr SDS PAGE : $\quad 98 \mathrm{kDa}$

Identified protein(s) : Lipoxygenase-2 (TC112465)

\begin{tabular}{|c|c|c|c|c|c|}
\hline $\exp$ & cal & ppm & seq & protein & notes \\
\hline 1511.913 & 1511.92 & 4 & IPLILSLDIYVPR & & \\
\hline 1533.901 & 1533.72 & 117 & LDGLTVDEAMNNNK & & \\
\hline 1549.855 & 1549.72 & 87 & LDGLTVDEAMNNNK & & $\mathrm{O}, \mathrm{M}$ \\
\hline 1713.822 & 1713.88 & 37 & DWVFPDQALPADLVK & & \\
\hline 1862.994 & 1863.06 & 38 & LFILNHHDVLIPYLR & & $\mathrm{V}$ \\
\hline 1862.994 & 1862.98 & 8 & MLGQIVGGLIGGHHDSKK & & N-term, O, M \\
\hline 1872.966 & 1873.04 & 41 & QILINAGGVLESTVFPSK & & M \\
\hline 1912.029 & 1912.09 & 32 & IPLILSLDIYVPRDER & & \\
\hline 2135.135 & 2135.12 & 8 & IFFANQPYLPSETPELLR & & \\
\hline 2308.205 & 2308.21 & 3 & LLIEDYPYAVDGLEIWSAIK & & $\mathrm{V}$ \\
\hline 2354.356 & 2354.28 & 32 & LTDIEKQIIQRNGDNILTNR & & M \\
\hline 2775.354 & 2775.43 & 27 & TAWRTDEEFAREMLAGVNPIIISR & & M \\
\hline 2970.364 & 2970.48 & 38 & FAMEMSAVVYKDWVFPDQALPADLVK & & \\
\hline 3031.326 & 3031.49 & 54 & SLTLEDVPNHGKVHFVCNSWVYPSFR & & \\
\hline
\end{tabular}

TC112465, Coverage: $25.0 \%(215 / 860)$

MLGQIVGGLIGGHHDSKKVKGTVVMMKKNALDFTDLAGSLTDKIFEALGQKVSFQLISSVQSDPANGLQGKHSNPAYLENFLF TLTPLAAGETAFGVTFDWNEEFGVPGAF I I KNTH INEFFLKSLTLEDVPNHGKVHFVCNSWVYPSFRYKS DRIFFANQPYLPS ETPELLRKYRENELLTLRGDGTGKREAWDRIYDYDVYNDLGNPDQGEQNVRTTLGGSADYPYPRRGRTGRPPTRTDPKSESRI PLILSLDIYVPRDERFGHLKMSDFLTYALKS IVQFILPELHALFDGTPNEFDSFEDVLRLYEGGIKLPQGPLFKALTAAIPLE MMKELLRTDGEGILRFPTPLVIKDSKTAWRTDEEFAREMLAGVNPIIISRLQEFP PKSKLDPEAYGNQNSTITAEHIEDKLDG LTVDEAMNNNKLFILNHHDVLI PYLRRINTTTTKTYASRTLLFLQDNGSLKPLAIELSLPHPDGDQFGVTSKVYTPSDQGVES S IWQLAKAYVAVNDS GVHQLIS HWLNTHAVIEPFVIATNRQLSVLHP I HKLLYPHFRDTMNINAMARQILINAGGVLESTVFP SKFAMEMSAVVYKDWVFPDQALPADLVKRGVAVEDSSSP HGVRLLIEDYPYAVDGLEIWSAIKSWVTDYCSFYYGSDEEILKD NELQAWWKELREVGHGDKKNEPWWPEMETPQELIDSCTTI IWIASALHAAVNFGQYPYAGYLPNRPTVSRRFMPEPGTPEYEE LKKNPDKAFLKTITAQLQTLLGVSLIEI LSRHTTDE IYLGQRES PEWTKDKEPLAAFDKFGKKLTDIEKQIIQRNGDNILTNR SGPVNAPYTLLFPTSEGGLTGKGIPNSVSI

Mr SDS PAGE : $\quad 90 \mathrm{kDa}$ Identified protein(s) : Lipoxygenase (TC112595)

\begin{tabular}{|c|c|c|c|c|c|}
\hline $\exp$ & cal & ppm & seq & protein & notes \\
\hline 1133.620 & 1133.58 & 32 & DKEPLAAFDK & & \\
\hline 1188.595 & 1188.60 & 2 & MSDFLTYALK & & \\
\hline 1275.616 & 1275.61 & 4 & FAMEMSAVVYK & & \\
\hline 1298.724 & 1298.75 & 20 & ALTAAIPLEMIR & & \\
\hline 1411.737 & 1411.69 & 37 & TTLGGSAEYPYPR & & \\
\hline 1511.920 & 1511.92 & 0 & IPLILSLDIYVPR & & \\
\hline 1711.863 & 1711.80 & 37 & DSKTAWRTDEEFAR & & M \\
\hline 1713.859 & 1713.88 & 15 & DWVFPDQALPADLVK & & \\
\hline 1863.016 & 1863.06 & 26 & LFILNHHDVLIPYLR & & \\
\hline 1872.938 & 1873.04 & 56 & QILINAGGVLESTVFPSK & & \\
\hline 2008.015 & 2008.00 & 6 & VYTPSDQGVESSIWQLAK & & \\
\hline
\end{tabular}




\begin{tabular}{llll}
2074.992 & 2075.08 & 41 & VSFQLISSVQGDPTNGLQGK \\
2206.294 & 2206.14 & 70 & SGPVNAPYTLLFPTSEGGLTGK \\
2308.302 & 2308.21 & 39 & LLIEDYPYAVDGLEIWSAIK \\
2502.305 & 2502.14 & 65 & IYDYDIYNDLGNPDEGKENVR \\
\hline
\end{tabular}

TC112595, Coverage: $27.0 \%(232 / 860)$

MIGQIKSGLFGP PDDSKKLKGTVVMMNKNALGFTDLAGSLTDTAFDVLGQKVSFQLISSVQGDPTNGLQGKHSNPAYLENSLF TLPPLTVDETSFGVTFDWNEEFGVPGAFI IKNTH INEFFLKSLTLEDVPNHGKVHFVCNSWVYPSFSYKSDRIFFVNQPYLPS KTPELLRKYRENELLTLRGDGTGKREAWDRIYDYDIYNDLGNPDEGKENVRTTLGGSAEYPYPRRGRTGRPPTRTDPKSESRI PLILSLDIYVPRDERFGHLKMSDFLTYALKS IVQF I LPELHALFDGTPNEFDSFEDVLRLYEGGIKLPQGPLFKALTAAIPLE MIRELLRTDGEGILRFPTPLVIKDSKTAWRTDEEFAREMLAGVNPVI I SRLQEFPPKSKLDPEAYGNQNSTITAEHIEDKLDG LTVDEAMNNNKLFILNHHDVLIPYLRRINTTTTKTYASRTLLFLQDNGSLKPLAIELSLPHPDGDQFGVTSKVYTPSDQGVES SIWQLAKAYVAVNDSGVHQLISHWLNTHAVIEP FVIATNRQLSVLHP I HKLLYPHFRDTMNINAMARQILINAGGVLESTVFP SKFAMEMSAVVYKDWVFPDQALPADLVKRGVAVEDS SSPHGVRLLIEDYPYAVDGLEIWSAIKSWVTDYCSFYYGSDEEILKD NELQAWWKELREVGHGDKKNEPWWPEMERPQELIDSCTTI IWIASALHAAVNFGQYPYAGYLPNRPTVSRRFMPEPGTPEYEE LKKNP DKAFLKTITAQLQTLLGVSLIEILSRHTTDE IYLGQRESPEWTKDKEPLAAFDKFGKKLTDIEKQI IQRNGDNILTNR SGPVNAPYTLLFPTSEGGLTGKGIPNSVSI

Mr SDS PAGE : $\quad 80 \mathrm{kDa}$

Identified protein(s) : Methionine synthase (TC119108)

\begin{tabular}{|c|c|c|c|c|c|}
\hline $\exp$ & cal & ppm & seq & protein & notes \\
\hline 1096.596 & 1096.58 & 16 & YLFAGVVDGR & & \\
\hline 1181.634 & 1181.63 & 2 & GVTAFGFDLVR & & \\
\hline 1469.836 & 1469.83 & 3 & AGITVIQIDEAALR & & \\
\hline 1469.836 & 1469.87 & 22 & VVEVNALAKALSGAK & & V \\
\hline 1658.882 & 1658.83 & 32 & YGAGIGPGVYDIHSPR & & \\
\hline 2071.876 & 2072.06 & 87 & EGVKYGAGIGPGVYDIHSPR & & \\
\hline 2135.137 & 2135.22 & 38 & AGITVIQIDEAALREGLPLR & & V \\
\hline 2135.137 & 2135.16 & 11 & TRKYTEVKPALQNMVSAAK & & M \\
\hline 2310.483 & 2310.31 & 76 & LNLPILPTTTIGSFPQTVELR & & \\
\hline 2983.413 & 2983.40 & 3 & WFDTNYHFIVPELGPDVNFSYASHK & & \\
\hline 3122.170 & 3121.76 & 131 & LDAQQKKLNLPILPTTTIGSFPQTVELR & & M \\
\hline
\end{tabular}

TC119108, Coverage: 19.3\% (148/765)

MASHVVGYPRMGPKRELKFALESFWDGKSSAEDLKKVSADLRSS IWKQMSDAGIKYIPSNTFSYYDQVLDTTAMLGAVPSRYN WTGGEIEFGTYF SMARGNASVPAMEMTKWFDTNYHFIVPELGPDVNFSYASHKAVNEYKEAKAQGVDTVPVLVGPVSYLLLSK PAKGVEKSFPLLSLLDKILPIYKEVIAELKAAGASWIQLDEPTLVLDLESHKLEAFTKAYADLESSLSGLNVLVETYFADVPA GAFKTLTALKGVTAFGFDLVRGTQTLELIKSSFP SGKYLFAGVVDGRN IWANDLAAS LALLQSLEGVVGKDKLVASTSCSLLH TAVDLINETKLDDEIKSWLAFAAQKVVEVNALAKALSGAKDEAFFSANAAAQASRKS SPRVTNEAVQKASAALQGSDHRRATN VSARLDAQQKKLNLPILPTTTIGSFPQTVELRRVRREYKAKKISEEEYVKAITEE IKKVVDLQEELDIDVLVHGEPERNDMVE YFGEQLSGFAFTANGWVQSYGSRCVKPPI IYGDVSRPKPMTVFWS SKAQEMTKRPMKGMLTGPVTILNWSFVRNDQPRFETCY QIALAIKDEVEDLEKAGITVIQIDEAALREGLPLRKAEHAFYLNWAVHS FRITNVGIEDTTQI HTHMCYSNFNDI I HS I I DMD ADVITIENSRSDEKLLSVFREGVKYGAGIGPGVYDIHSPRI PSTEEIADRVNKMLAVLDTNILWVNPDCGLKTRKYTEVKPAL QNMVSAAKTIRTQLASAK

Mr SDS PAGE : $\quad 75 \mathrm{kDa}$ Identified protein(s) : Lipoxygenase (TC112595)

\begin{tabular}{llllll}
\hline exp & cal & ppm & seq & protein & notes \\
\hline 1133.680 & 1133.58 & 85 & DKEPLAAFDK & \\
1189.667 & 1189.56 & 87 & DNELQAWWK & \\
1298.769 & 1298.75 & 15 & ALTAAIPLEMIR & \\
1398.753 & 1398.78 & 17 & EMLAGVNPVIISR &
\end{tabular}




\begin{tabular}{llll}
1511.792 & 1511.92 & 84 & IPLILSLDIYVPR \\
1713.759 & 1713.88 & 73 & DWVFPDQALPADLVK \\
1862.995 & 1863.06 & 37 & LFILNHHDVLIPYLR \\
1869.911 & 1869.99 & 40 & DWVFPDQALPADLVKR \\
1873.039 & 1873.04 & 2 & QILINAGGVLESTVFPSK \\
2007.997 & 2008.00 & 3 & VYTPSDQGVESSIWQLAK \\
2247.192 & 2247.14 & 21 & TDEEFAREMLAGVNPVIISR \\
2308.283 & 2308.21 & 31 & LLIEDYPYAVDGLEIWSAIK \\
2502.175 & 2502.14 & 13 & IYDYDIYNDLGNPDEGKENVR \\
2690.490 & 2690.58 & 33 & LPQGPLFKALTAAPILEMIRELLR \\
\hline
\end{tabular}

TC112595, Coverage: $20 \%(172 / 860)$

MIGQIKSGLFGP PDSKKLKGTVVMMNKNALGFTDLAGSLTDTAFDVLGQKVSFQLISSVQGDPTNGLQGKHSNPAYLENSLF TLPPLTVDETSFGVTFDWNEEFGVPGAFI IKNTH INEFFLKSLTLEDVPNHGKVHFVCNSWVYPSFSYKSDRIFFVNQPYLPS KTPELLRKYRENELLTLRGDGTGKREAWDRIYDYDIYNDLGNPDEGKENVRTTLGGSAEYPYPRRGRTGRPPTRTDPKSESRI PLILSLDIYVPRDERFGHLKMSDFLTYALKS IVQFILPELHALFDGTPNEFDSFEDVLRLYEGGIKLPQGPLFKALTAAIPLE MIRELLRTDGEGILRFPTPLVIKDSKTAWRTDEEFAREMLAGVNPVIISRLQEFP PKSKLDPEAYGNQNSTITAEHIEDKLDG LTVDEAMNNNKLFILNHHDVLIPYLRRINTTTTKTYASRTLLFLQDNGSLKPLAIELSLPHPDGDQFGVTSKVYTPSDQGVES SIWQLAKAYVAVNDSGVHQLISHWLNTHAVIEPFVIATNRQLSVLHP I HKLLYPHFRDTMNINAMARQILINAGGVLESTVFP SKFAMEMSAVVYKDWVFPDQALPADLVKRGVAVEDSSSPHGVRLLIEDYPYAVDGLEIWSAIKSWVTDYCSFYYGSDEEILKD NELQAWWKELREVGHGDKKNEPWWPEMERPQELIDSCTTI IWIASALHAAVNFGQYPYAGYLPNRPTVSRRFMPEPGTPEYEE LKKNPDKAFLKTITAQLQTLLGVSLIEI LSRHTTDEIYLGQRESPEWTKDKEPLAAFDKFGKKLTDIEKQI IQRNGDNILTNR SGPVNAPYTLLFPTSEGGLTGKGIPNSVSI

Mr SDS PAGE : $\quad 68 \mathrm{kDa}$ Identified protein(s) : Lipoxygenase (TC112595)

\begin{tabular}{|c|c|c|c|c|c|}
\hline $\exp$ & cal & ppm & seq & protein & notes \\
\hline 1133.587 & 1133.58 & 3 & DKEPLAAFDK & & \\
\hline 1188.575 & 1188.60 & 18 & MSDFLTYALK & & \\
\hline 1189.511 & 1189.56 & 44 & DNELQAWWK & & \\
\hline 1298.696 & 1298.75 & 42 & ALTAAIPLEMIR & & \\
\hline 1713.839 & 1713.88 & 27 & DWVFPDQALPADLVK & & \\
\hline 1869.905 & 1869.99 & 43 & DWVFPDQALPADLVKR & & \\
\hline 2008.068 & 2008.00 & 33 & VYTPSDQGVESSIWQLAK & & \\
\hline 2206.108 & 2206.14 & 14 & SGPVNAPYTLLFPTSEGGLTGK & & \\
\hline 2247.229 & 2247.14 & 38 & TDEEFAREMLAGVNPVIISR & & \\
\hline 2345.248 & 2345.09 & 68 & LDPEAYGNQNSTITAEHIEDK & & \\
\hline 2502.321 & 2502.14 & 72 & IYDYDIYNDLGNPDEGKENVR & & \\
\hline
\end{tabular}

TC112595, Coverage: $18.5 \%(159 / 860)$

MIGQIKSGLFGPPDDSKKLKGTVVMMNKNALGFTDLAGSLTDTAFDVLGQKVSFQLISSVQGDPTNGLQGKHSNPAYLENSLF TLPPLTVDETSFGVTFDWNEEFGVPGAFIIKNTH INEFFLKSLTLEDVPNHGKVHFVCNSWVYPSFSYKSDRIFFVNQPYLPS KTPELLRKYRENELLTLRGDGTGKREAWDRIYDYDIYNDLGNPDEGKENVRTTLGGSAEYPYPRRGRTGRPPTRTDPKSESRI PLILSLDIYVPRDERFGHLKMSDFLTYALKS IVQFILPELHALFDGTPNEFDSFEDVLRLYEGGIKLPQGPLFKALTAAIPLE MIRELLRTDGEGILRFPTPLVIKDSKTAWRTDEEFAREMLAGVNPVIISRLQEFP PKSKLDPEAYGNQNSTITAEHIEDKLDG LTVDEAMNNNKLFILNHHDVLI PYLRRINTTTTKTYASRTLLFLQDNGSLKPLAIELSLPHPDGDQFGVTSKVYTPSDQGVES SIWQLAKAYVAVNDSGVHQLIS HWLNTHAVIEPFVIATNRQLSVLHP I HKLLYPHFRDTMNINAMARQ I L INAGGVLESTVFP SKFAMEMSAVVYKDWVFPDQALPADLVKRGVAVEDSSSPHGVRLLIEDYPYAVDGLEIWSAIKSWVTDYCSFYYGSDEEILKD NELQAWWKELREVGHGDKKNEPWWPEMERPQELIDSCTTI IWIASALHAAVNFGQYPYAGYLPNRPTVSRRFMPEPGTPEYEE LKKNPDKAFLKTITAQLQTLLGVSLIEI LSRHTTDE IYLGQRES PEWTKDKEPLAAFDKFGKKLTDIEKQI IQRNGDNI LTNR SGPVNAPYTLLFPTSEGGLTGKGIPNSVS I

Mr SDS PAGE : $\quad 60 \mathrm{kDa}$ 
Identified protein(s) : Enolase (TC112026)

\begin{tabular}{|c|c|c|c|c|c|}
\hline $\exp$ & cal & ppm & seq & protein & notes \\
\hline 1579.945 & 1579.92 & 18 & AVNNVNSIIGPALIGK & & \\
\hline 1804.937 & 1804.94 & 4 & AAVPSGASTGIYEALELR & & \\
\hline 1827.875 & 1827.88 & 0 & IEEELGSEAVYAGASFR & & \\
\hline 1896.964 & 1896.96 & 4 & GNPTVEVDVHVSNGVFAR & & \\
\hline 1919.848 & 1920.01 & 84 & VNQIGSVTESIEAVKMSK & & M \\
\hline 2252.100 & 2252.13 & 13 & SGETEDTFIADLAVGLSTGQIK & & \\
\hline 2324.042 & 2324.04 & 0 & YGQDATNVGDEGGFAPNIQENK & & \\
\hline 2382.244 & 2382.28 & 14 & LTTEIGEQVQIVGDDLLVTNPK & & \\
\hline 2980.373 & 2980.34 & 11 & SFVSEYPIVSIEDPFDQDDWETYAK & & \\
\hline
\end{tabular}

TC112026, Coverage: $40.1 \%(178 / 444)$

MATIKS IKARQI FDSRGNPTVEVDVHVSNGVFARAAVPSGASTGIYEALELRDGGSDYLGKGVSKAVNNVNSIIGPALIGKDP TDQTGLDNFMVHELDGTQNEWGWCKEKLGANA I LAVSLAVCKAGAAVRNVPLYKHIADLAGNKKLVLPVPAFNVINGGSHAGN KLAMQEFMI LPVGAANFKEAMKMGCEVYHHLKSVIKKKYGQDATNVGDEGGFAPNIQENKEGLELLKTAIEKAGYTGKVVIGM DVAASEFYGKDKSYDLNFKEESNDGSQKISGDQLKDLYKSFVSEYPIVS IEDPFDQDDWETYAKLTTEIGEQVQIVGDDLLVT NPKRVAKAIAEKTCNALLLKVNQIGSVTESIEAVKMSKKAGWGVMTSHRSGETEDTFIADLAVGLSTGQIKTGAPCRSERLAK YNQLLRIEEELGSEAVYAGASFRKPVEPY

Mr SDS PAGE : $\quad 42 \mathrm{kDa}$

Identified protein(s) : Patatin 3-k1 (Kuras, DQ114421)

Represented : Patatin 1-k1 (Kuras, DQ114415)

Patatin 1-k2 (Kuras, DQ114416)

Patatin 2-k1 (Kuras, DQ114417)

\begin{tabular}{|c|c|c|c|c|c|}
\hline $\exp$ & cal & ppm & seq & protein & notes \\
\hline 1133.701 & 1133.65 & 45 & YLLQVLQEK & Pat2-k1 & Unique \\
\hline 1151.677 & 1151.61 & 58 & YLMQVLQEK & Pat3-k1, Pat1-k1, Pat1-k2 & \\
\hline 1167.721 & 1167.61 & 95 & YLMQVLQEK & Pat3-k1, Pat1-k1, Pat1-k2 & $\mathrm{O}, \mathrm{M}$ \\
\hline 1289.733 & 1289.67 & 49 & LAQEDPAFASIK & Pat3-k1 & Unique \\
\hline 1305.739 & 1305.66 & 60 & LAQEDPAFSSIK & Pat2-k1 & Unique \\
\hline 1437.825 & 1437.68 & 101 & DSPETYEEALKR & Pat2-k1 & Unique \\
\hline 1448.776 & 1448.71 & 45 & DIIPFYFDHGPK & Pat3-k1 & Unique \\
\hline 1499.838 & 1499.78 & 39 & IFEPSGFHLVEPK & Pat3-k1 & Unique \\
\hline 1705.925 & 1705.80 & 73 & TLGEMVTVLSIDGGGIK & Pat3-k1, Pat1-k1, Pat2-k1 & $\mathrm{O}$ \\
\hline 1720.880 & 1720.80 & 47 & SVSEDNHETYEVALK & Pat3-k1 & Unique \\
\hline 1757.961 & 1757.94 & 12 & VHQALTEVAISSFDIK & Pat3-k1, Pat1-k1, Pat1-k2, Pat2-k1 & \\
\hline 1825.974 & 1825.93 & 24 & QMLLLSLGTGTNSEFAK & Pat3-k1 & $\mathrm{O}$, Unique, $\mathrm{M}$ \\
\hline 1864.872 & 1864.89 & 10 & WGILQWMSPLWEMR & Pat3-k1 & $2 \mathrm{O}$, Unique, $\mathrm{M}$ \\
\hline 2247.094 & 2247.08 & 6 & ADDASEANMELLAQVGENLLK & Pat1-k2 & $\mathrm{O}$, Unique \\
\hline 2275.121 & 2275.11 & 5 & ADDASEANMELLVQVGENLLK & Pat1-k1 & \\
\hline 2807.316 & 2807.42 & 37 & SVSEDNHETYEVALKRFAKLLSDR & Pat3-k1 & Unique \\
\hline
\end{tabular}

DQ114421, Coverage: $36.9 \%(134 / 363)$

TLGEMVTVLSIDGGGIKGI I PAT I LEFLEGQLQEVDNNTDARLADYFDVIGGTGTGGLLTAMITTPNENNRP FAAAKDIIPFY FDHGPKIFEPSGFHLVEPKYDGKYLMQVLQEKLGETRVHQALTEVAISSFDIKTNKPVIFTKSNLAKTPELDAKMYDICYSTA AAPTYFP PHYFATNTSNGDQYDFNLVDGDVAAVDPS LLS ISVATRLAQEDPAFAS IKSLNYKQMLLLSLGTGTNSEFAKNYTA EEAAKWGILQWMSPLWEMRSAAS SYMNDYYLSTVFQALDSQNNYL $\overline{\text { RVQENALTGTATTFDDASVANMILLVQVGENLLKKSVS }}$ EDNHETYEVALKRFAKLLSDRKKLRANKASF 
DQ114415, Coverage: $17.3 \%(63 / 364)$

TLGEMVTVLS IDGGGIKGI IPGI I LEFLEGQLQKMDNNADARLADY FDVIGGTSTGGLLTAMITTPNENNRPFAAAKDIVPFY FQHGPH I FNSSTGQFFGPKYDGKYLMQVLQEKLGETRVHQALTEVAISSFDIKTNKPVI FTKSNLAKSPELDAKMSDICYSTA AAPTYFP PHYFATNTSNGDKYEFNLVDGAVATVADPALLSVSVATRRAEEDPAFAS IRSLNYKQLLLLSLGTGTNSEFDKTHT AQETAKWGALQWMLVIQQMTEAASSYMTDYYLSTVFQDLHSQNNYLRVQENALTGTTTKADDASEANMELLVQVGENLLKKPV SKDNPETYEEALKRFAKLLSDRKKFRANKASY

DQ114416, Coverage: 12.6\% (46/364)

SLEEMVTVLS I DGGG IKGI I PGT I LEFLEGQLQKMDNNADARLADY FDVIGGTSTGGLLTAMITT PNENNRPFAAANEIVPFY FEHGPH I FNSSTGQFFGPKYDGKYLMQVLQEKLGETRVHQALTEVAISSFDIKTNKPVIFTKSNLAKSPELDAKMYDICYSTA AAPTYFPPHYFATNTINGDKYKFNLVDGAVATVADPALLSVSVATRRAQEDPAFAS IRS LNYKKMLLLSLGTGTTSEFDKTHT AEETAKWGALQWMLVIQQMTEAAS SYMTDYYLSTVFQDLHSQNNYLRVQENALTGTTTKADDASEANMELLAQVGENLLKKPV SKDNPETYEEALKRFAKLLSDRKKLRANKASY

DQ114417, Coverage: $18.2 \%(66 / 363)$

TLGEMVTVLS IDGGGIKGI I PAT I LEFLEGQLQEVDNNKDARLADY FDVIGGTSTGGLLTAMITTPNENNRPFAAAKDIVPFY FEHGPH I FNS S GT I FGPMY DGKYLLQVLQEKLGETRVHQALTEVAISSFDIKTNKPVIFTKSNLAKS PE LDAKMYDICYSTAA APIYFPPHYFVTHTSNGDRYEFNLVDGAVATVGDPALLSLSVATRLAQEDPAFSSIKSLDYKQMLLLSLGTGTNSEFDKTYTA EEAAKWGPLRWLLAIQQMTNAAS SYMTDYYLSTVFQARHSQNNYLRVQENALTGTTTEMDDASEANMELLVQVGETLLKKPVS KDSPETYEEALKRFAKLLSDRKKLRANKASY

Mr SDS PAGE : $\quad 40 \mathrm{kDa}$

Identified protein(s) : Patatin 2-k1 (Kuras, DQ114417)

Represented : Patatin1-k1 (Kuras, DQ114415)

Patatin1-k2 (Kuras, DQ114416)

Patatin2-k3 (Kuras, DQ114418)

\begin{tabular}{|c|c|c|c|c|c|}
\hline $\exp$ & cal & ppm & seq & protein & notes \\
\hline 1133.683 & 1133.65 & 29 & YLLQVLQEK & Pat2-k1 & Unique \\
\hline 1167.636 & 1167.61 & 22 & YLMQVLQEK & Pat1-k1, Pat1-k2 & $\mathrm{O}, \mathrm{M}$ \\
\hline 1262.735 & 1262.65 & 67 & VQENALTGTTTK & Pat1-k1, Pat1-k2 & \\
\hline 1592.886 & 1592.80 & 54 & TYTAEEAAKWGPLR & Pat2-k1, Pat2-k3 & \\
\hline 1689.946 & 1689.90 & 27 & TLGEMVTVLSIDGGGIK & Pat1-k1, Pat2-k1, Pat2-k3 & N-term \\
\hline 1689.946 & 1689.94 & 4 & YLLQVLQEKLGETR & Pat2-k1 & Unique \\
\hline 1705.932 & 1705.90 & 19 & TLGEMVTVLSIDGGGIK & Pat1-k1, Pat2-k1, Pat2-k3 & $\mathrm{O}, \mathrm{N}$-term \\
\hline 1757.965 & 1757.94 & 14 & VHQALTEVAISSFDIK & Pat1-k1, Pat1-k2, Pat2-k1, Pat2-k3 & \\
\hline 1836.287 & 1835.97 & 173 & QLLLLSLGTGTNSEFDK & Pat1-k1 & Unique \\
\hline 1856.017 & 1856.05 & 18 & GIIPGTILEFLEGQLQK & Pat1-k2 & Unique \\
\hline 1869.983 & 1869.92 & 34 & QMLLLSLGTGTNSEFDK & Pat2-k1, Pat2-k3 & $\mathrm{O}, \mathrm{M}$ \\
\hline 2231.117 & 2231.08 & 16 & ADDASEQNMELLAQVGENLLK & Pat1-k2 & Unique \\
\hline 2247.091 & 2247.08 & 5 & ADDASEQNMELLAQVGENLLK & Pat1-k2 & $\mathrm{O}$, Unique, $\mathrm{M}$ \\
\hline 2259.106 & 2259.11 & 2 & ADDASEANMELLVQVGENLLK & Pat1-k1 & Unique \\
\hline 2275.133 & 2275.11 & 10 & ADDASEANMELLVQVGENLLK & Pat1-k1 & $\mathrm{O}$, Unique, $\mathrm{M}$ \\
\hline 2441.358 & 2441.28 & 32 & SLNYKQLLLLSLGTGTNSEFDK & Pat1-k1 & Unique \\
\hline 2441.358 & 2441.28 & 32 & GIIPATILEFLEGQLQEVDNNK & Pat2-k1, Pat2-k3 & \\
\hline 2783.421 & 2783.45 & 10 & GIIPATILEFLEGQLQEVDNNKDAR & Pat2-k1, Pat2-k3 & \\
\hline 3241.337 & 3241.52 & 56 & VQENALTGTTTEMDDASEANMELLVQVGEK & Pat2-k3 & $\mathrm{O}$, Unique \\
\hline 3257.252 & 3257.52 & 82 & VQENALTGTTTEMDDASEANMELLVQVGEK & Pat2-k3 & $2 \mathrm{O}$, Unique \\
\hline 3289.742 & 3290.74 & 303 & WILAIQQMTNAASSYMTDYYLSTVFQAR & Pat2-k3 & $\mathrm{O}$, Unique \\
\hline 3305.834 & 3306.73 & 271 & WLLAIQQMTNAASSYMTDYYLSTVFQAR & Pat2-k1 & $2 \mathrm{O}$, Unique, $\mathrm{M}$ \\
\hline
\end{tabular}

DQ114417, Coverage: 36.1\% (131/363) 
TLGEMVTVLSIDGGGIKGI IPATILEFLEGQLQEVDNNKDARLADYFDVIGGTSTGGLLTAMITTPNENNRPFAAAKDIVPFY FEHGPH I FNSSGT IFGPMYDGKYLLQVLQEKLGETRVHQALTEVAISSFDIKTNKPVIFTKSNLAKSPELDAKMYDICYSTAA APIYFPPHYFVTHTSNGDRYEFNLVDGAVATVGDPALLSLSVATRLAQEDPAFSSIKSLDYKQMLLLSLGTGTNSEFDKTYTA EEAAKWGPLRWLLAIQQMTNAASSYMTDYYLSTVFQARHSQNNYLRVQENALTGTTTEMDDASEANMELLVQVGETLLKKPVS KDSPETYEEALKRFAKLLSDRKKLRANKASY

DQ114415, Coverage: $26.6 \%(97 / 364)$

TLGEMVTVLSIDGGGIKGI IPGI ILEFLEGQLQKMDNNADARLADYFDVIGGTSTGGLLTAMITTPNENNRPFAAAKDIVPFY FQHGPHIFNSSTGQFFGPKYDGKYLMQVLQEKLGETRVHQALTEVAISSFDIKTNKPVIFTKSNLAKSPELDAKMSDICYSTA AAPTYFPPHYFATNTSNGDKYEFNLVDGAVATVADPALLSVSVATRRAEEDPAFAS IRSLNYKQLLLLSLGTGTNSEFDKTHT AQETAKWGALQWMLVIQQMTEAASSYMTDYYLSTVFQDLHSQNNYLRVQENALTGTTTKADDASEANMELLVQVGENLLKKPV SKDNPETYEEALKRFAKLLSDRKKFRANKASY

DQ114416, Coverage: $14.8 \%(54 / 364)$

SLEEMVTVLS I DGGG I KGI IPGTILEFLEGQLQKMDNNADARLADYFDVIGGTSTGGLLTAMITTPNENNRP FAAANE IVPFY FEHGPHIFNSSTGQFFGPKYDGKYLMQVLQEKLGETRVHQALTEVAISSFDIKTNKPVIFTKSNLAKSPELDAKMYDICYSTA AAPTYFPPHYFATNTINGDKYKFNLVDGAVATVADPALLSVSVATRRAQEDPAFAS IRSLNYKKMLLLSLGTGTTSEFDKTHT AEETAKWGALQWMLVIQQMTEAAS SYMTDYYLSTVFQDLHSQNNYLRVQENALTGTTTKADDASEANMELLAQVGENLLKKPV SKDNPETYEEALKRFAKLLSDRKKLRANKASY

DQ114418, Coverage: 40.5\% (147/363)

TLGEMVTVLSIDGGGIKGI IPATILEFLEGQLQEVDNNKDARLADYFDVIGGTSTGGLLTAMITTPNENNRPFAAAKDIVPFY FEHGPHIFNSSGS IFGPMYDGKYFLQVLQEKLGETRVHQALTEVAISSFDIKTNKPVIFTKSNLAKSPELDAKMYDICYSTAA APTYFPPHYFVTHTSNGDKYEFNLVDGAVATVGDPALLSLSVATKLAQVDPKFAS IKSLNYKQMLLLSLGTGTNSEFDKTYTA EEAAKWGPLRWILAIQQMTNAASSYMTDYYLSTVFQARHS QNNYLRVQENALTGTTTEMDDASEANMELLVQVGEKLLKKPVS KDSPETYEEALKRFAKLLSDRKKLRANKASY

Mr SDS PAGE : $\quad 39 \mathrm{kDa}$

Identified protein(s) : Patatin 2-k1 (Kuras, DQ114417)

Represented : Patatin1-k1 (Kuras, DQ114415)

Patatin1-k2 (Kuras, DQ114416)

\begin{tabular}{llllll}
\hline exp & cal & ppm & seq & protein & notes \\
\hline 1133.655 & 1133.65 & 5 & YLLQVLQEK & Pat2-k1 & Unique \\
1167.637 & 1167.61 & 23 & YLMQVLQEK & Pat1-k1, Pat1-k2 & O, M \\
1305.650 & 1305.66 & 8 & LAQEDPAFSSIK & Pat2-k1 & Unique \\
1437.675 & 1437.68 & 3 & DSPETYEEALKR & Pat2-k1 & Pat1-k1, Pat2-k1 \\
1689.8702 & 1689.90 & 17 & TLGEMVTVLSEDGGGIK & Pat2-k1 & Unique \\
1689.870 & 1689.95 & 47 & YLLQVLQEKLGETR & Pat1-k1, Pat2-k1 & O \\
1705.872 & 1705.90 & 16 & TLGEMVTVLSEDGGGIK & Pat1-k1, Pat1-k2, Pat2-k1 & Unique \\
1757.882 & 1757.90 & 10 & VHQALTEVAISSFDIK & Pat1-k1 & O, Unique, M \\
1835.920 & 1835.97 & 27 & QLLLLSLGTGTNSEFDK & Pat2-k1 & Unique \\
1869.876 & 1869.92 & 23 & QMLLLSLGTGTNSEFDK & Pat1-k2 & Unique \\
2247.126 & 2247.08 & 21 & ADDASEANMELLAQVGENLLK & Pat1-k1 & Unique \\
2441.328 & 2441.28 & 20 & SLNYKQLLLLSLGTGTNSEFDK & Pat2-k1 & O, Unique, M \\
2783.587 & 2783.45 & 49 & GIIPATILEFLEGQLQEVDNNKDAR & Pat2-k1 & \\
3305.880 & 3305.74 & 42 & WLLAIQQMTNAASSYMTDYYLSTVFQAR & & \\
\hline
\end{tabular}

DQ114417, Coverage: $38.8 \%(141 / 363)$

TLGEMVTVLSIDGGGIKGI IPATILEFLEGQLQEVDNNKDARLADYFDVIGGTSTGGLLTAMITTPNENNRPFAAAKDIVPFY FEHGPH I FNSSGT IFGPMYDGKYLLQVLQEKLGETRVHQALTEVAISSFDIKTNKPVIFTKSNLAKSPELDAKMYDICYSTAA APIYFPPHYFVTHTSNGDRYEFNLVDGAVATVGDPALLSLSVATRLAQEDPAFSSIKSLDYKQMLLLSLGTGTNSEFDKTYTA EEAAKWGPLRWLLAIQQMTNAASSYMTDYYLSTVFQARHSQNNYLRVQENALTGTTTEMDDASEANMELLVQVGETLLKKPVS KDSPETYEEALKREAKLLSDRKKLRANKASY 
DQ114415, Coverage: 17.6\% (64 / 364)

TLGEMVTVLS IDGGGIKGI I PGI I LEFLEGQLQKMDNNADARLADY FDVIGGTSTGGLLTAMITTPNENNRPFAAAKDIVPFY FQHGPH I FNSSTGQFFGPKYDGKYLMQVLQEKLGETRVHQALTEVAISSFDIKTNKPVI FTKSNLAKSPELDAKMSDICYSTA AAPTYFPPHYFATNTSNGDKYEFNLVDGAVATVADPALLSVSVATRRAEEDPAFAS IRSLNYKQLLLLSLGTGTNSEFDKTHT AQETAKWGALQWMLVIQQMTEAASSYMTDYYLSTVFQDLHSQNNYLRVQENALTGTTTKADDASEANMELLVQVGENLLKKPV SKDNPETYEEALKRFAKLLSDRKKFRANKASY

DQ114416, Coverage: $12.6 \%(46 / 364)$

SLEEMVTVLS I DGGG IKGI I PGT I LEFLEGQLQKMDNNADARLADY FDVIGGTSTGGLLTAMITTPNENNRPFAAANEIVPFY FEHGPH I FNSSTGQFFGPKYDGKYLMOVLQEKLGETRVHQALTEVAISSFDIKTNKPVIFTKSNLAKSPELDAKMYDICYSTA AAPTYFP PHYFATNT INGDKYKFNLVDGAVATVADPALLSVSVATRRAQEDPAFAS IRS LNYKKMLLLSLGTGTTSEFDKTHT AEETAKWGALQWMLVIQQMTEAASSYMTDYYLSTVFQDLHSQNNYLRVQENALTGTTTKADDASEANMELLAQVGENLLKKPV SKDNPETYEEALKRFAKLLSDRKKLRANKASY

Mr SDS PAGE : $\quad 33 \mathrm{kDa}$

Identified protein(s) : Annexin p34 (TC119057)

\begin{tabular}{|c|c|c|c|c|c|}
\hline $\exp$ & cal & ppm & seq & protein & notes \\
\hline 1035.604 & 1035.67 & 61 & LIISILAHR & & \\
\hline 1146.620 & 1146.69 & 59 & LLVPLVSSYR & & \\
\hline 1274.721 & 1274.78 & 49 & KLLVPLVSSYR & & \\
\hline 1441.732 & 1441.80 & 51 & LVLIWTLDPSER & & \\
\hline 1514.673 & 1514.74 & 42 & QTYAETFGEDLLK & & \\
\hline 1576.707 & 1576.79 & 50 & QLEDEDEFVALLR & & \\
\hline 1720.820 & 1720.91 & 50 & GLVYPEHYFVEVLR & & \\
\hline 2335.182 & 2335.16 & 11 & AQLNATLNHYKDEYGEDILK & & \\
\hline
\end{tabular}

TC119057, Coverage: 29.3\% (92/314)

MASLTVPAEVPSVAEDCEQLRSAFKGWGTNEKLIISILAHRNAAQRKLIRQTYAETFGEDLLKELDRELTHDFEKLVLIWTLD PSERDAYLAKEATKRWTKSNFVLVEIACTRSPKELVLAREAYHARNKKSLEEDVAYHTTGDHRKLLVPLVSSYRYGGDEVDLR LAKAESKVLHEKI SDKAYSDDEV IRI LATRSKAQLNATLNHYKDEYGEDILKQLEDEDEFVALLRAT IKGLVYPEHYFVEVLR DAINRRGTEEDHLSRVIATRAEVDLKTIANEYQKRDSI PLGRAIAKDTGGDYENMLVALLGQEEE

\section{Fraction VI}

Mr SDS PAGE : $\quad 98 \mathrm{kDa}$ Identified protein(s) : 9-Lipoxygenase (TC112798)

\begin{tabular}{|c|c|c|c|c|c|}
\hline $\exp$ & cal & ppm & seq & protein & notes \\
\hline 858.447 & 858.47 & 30 & LQEFPPK & & \\
\hline 899.506 & 899.53 & 32 & LPQGPLFK & & \\
\hline 987.524 & 987.55 & 23 & ENELLTLR & & \\
\hline 1016.469 & 1016.51 & 42 & NGDNILTNR & & \\
\hline 1189.562 & 1189.56 & 1 & DNELQAWWK & & \\
\hline 1396.661 & 1396.68 & 15 & GVAVEDSSSPHGVR & & \\
\hline 1411.667 & 1411.69 & 13 & TTLGGSAEYPYPR & & \\
\hline 1412.769 & 1412.79 & 17 & EMLAGVNPIIISR & & \\
\hline 1424.730 & 1424.76 & 19 & IFFANQPYLPSK & & \\
\hline 1428.736 & 1428.79 & 36 & EMLAGVNPIIISR & & $\mathrm{O}, \mathrm{M}$ \\
\hline 1434.778 & 1434.81 & 20 & KYRENELLTLR & & $\mathrm{M}$ \\
\hline 1510.994 & 1510.82 & 121 & RINTTITKSYASR & & M \\
\hline 1533.707 & 1533.72 & 9 & LDGLTVDEAMNNNK & & \\
\hline
\end{tabular}




\begin{tabular}{lllll}
1549.675 & 1549.72 & 27 & LDGLTVDEAMNNNK & $\mathrm{O}, \mathrm{M}$ \\
1912.094 & 1912.09 & 2 & IPLLLSLDIYVPRDER & \\
1971.188 & 1971.13 & 31 & SESRIPLLLSLDIYVPR & $\mathrm{M}$ \\
2002.915 & 2002.90 & 6 & IYDYDIYNDLGNPDQGK & $\mathrm{M}$ \\
2206.231 & 2206.14 & 42 & SGPVNAPYTLLFPTSEGGLTGK & \\
2345.090 & 2345.09 & 1 & LDPEAYGNQNSTITAEHIEDK & \\
2501.262 & 2501.16 & 42 & IYDYDIYNDLGNPDQGKENVR & \\
\hline
\end{tabular}

TC112798, Coverage: $24.0 \%(207 / 864)$

MNIGQIMGGRELFGGHDDSKKVKGTVVMMKKNALDFTDLAGSLTDKAFDVLGQKVSFQLISSVQGDPTNGLQGKHSNPAYLEN SLFTLTPLTAGSETAFGVTFDWNEEFGVPGAFI IKNTH INEFFLKSLTLEDVPNHGKVHFDCNSWVYPSFRYKSDRIFFANQP YLPSKTPELLRKYRENELLTLRGDGTGKREAWDRIYDYDIYNDLGNPDQGKENVRTTLGGSAEYPYPRRGRTGRPPTRTDPKS ESRIPLLLSLDIYVPRDERFGHLKMSDFLTYALKS IVQF I LPELHALFDGTPNEFDSFEDVLRLYEGG IKLPQGPLFKALTAA IPLEMIKELLRTDGEGILRFPTPLVIKDSKTAWRTDEEFAREMLAGVNPIIISRLQEFPPKSKLDPEAYGNQNSTITAEHIED KLDGLTVDEAMNNNKLFILNHHDLLIPYLRRINTTITKSYASRTLLFLQDNGS LKPLAIELSLPHPDGDQFGVTSKVYTPSDQ GVESS IWQLAKAYVAVNDAGVHQLISHWLNTHAVIEPFVIATNRQLSVLHP I HKLLYPHFRDTMNINASARQILINAGGVLES TVFQSKFALEMSAVVYKDWVFPDQALPADLVKRGVAVEDSSSPHGVRLLIEDYPYAVDGLEIWSAIKSWVTDYCSFYYGSDEE ILKDNELQAWWKELREVGHGDKKNEPWWPEMETPQELIDSCTTI IWIASALHAAVNFGQYPYAGYLPNRPTVSRRFMPEPGTP EYEELKKNPDKAFLKTITAQLQTLLGVSLVEILSRHTTDE I YLGQRESPEWTKDKEPLAAFDKFGKKLTDIEKQI IQRNGDNI LTNRSGPVNAPYTLLFPTSEGGLTGKGIPNSVS I

Mr SDS PAGE : $\quad 57 \mathrm{kDa}$ Identified protein(s) : Enolase (TC112026)

\begin{tabular}{|c|c|c|c|c|c|}
\hline $\exp$ & cal & ppm & seq & protein & notes \\
\hline 1138.671 & 1138.61 & 54 & TAIEKAGYTGK & & \\
\hline 1258.623 & 1258.73 & 85 & AGAAVRNVPLYK & & \\
\hline 1490.849 & 1490.84 & 6 & SERLAKYNQLLR & & M \\
\hline 1579.945 & 1579.92 & 18 & AVNNVNSIIGPALIGK & & \\
\hline 1804.937 & 1804.94 & 4 & AAVPSGASTGIYEALELR & & \\
\hline 1827.875 & 1827.88 & 0 & IEEELGSEAVYAGASFR & & \\
\hline 1896.964 & 1896.96 & 4 & GNPTVEVDVHVSNGVFAR & & \\
\hline 1919.848 & 1920.01 & 4 & VNQIGSVTESIEAVKMSK & & M \\
\hline 1951.199 & 1951.13 & 35 & GVSKAVNNVNSIIGPALIGK & & \\
\hline 2252.100 & 2252.13 & 13 & SGETEDTFIADLAVGLSTGQIK & & \\
\hline 2324.042 & 2324.04 & 0 & YGQDATNVGDEGGFAPNIQENK & & \\
\hline 2382.244 & 2382.28 & 14 & LTTEIGEQVQIVGDDLLVTNPK & & \\
\hline 2406.291 & 2406.20 & 38 & AGYTGKVVIGMDVAASEFYGKDK & & M \\
\hline 2980.373 & 2980.34 & 11 & SFVSEYPIVSIEDPFDQDDWETYAK & & \\
\hline
\end{tabular}

TC112026, Coverage: $52.7 \%(234 / 444)$

MATIKS IKARQ I EDSRGNPTVEVDVHVSNGVFARAAVPSGASTGIYEALELRDGGSDYLGKGVSKAVNNVNSIIGPALIGKDP TDQTGLDNFMVHELDGTQNEWGWCKEKLGANA I LAVSLAVCKAGAAVRNVPLYKH IADLAGNKKLVLPVPAFNVINGGSHAGN KLAMQE FMI LPVGAANFKEAMKMGCEVYHHLKSVI KKKYGQDATNVGDEGGFAPNIQENKEGLELLKTAIEKAGYTGKVVIGM DVAASEFYGKDKSYDLNFKEESNDGSQKISGDQLKDLYKSFVSEYPIVSIEDPFDQDDWETYAKLTTEIGEQVQIVGDDLLVT NPKRVAKAIAEKTCNALLLKVNQIGSVTES IEAVKMSKKAGWGVMTSHRSGETEDTFIADLAVGLSTGQIKTGAPCRSERLAK YNQLLRIEEELGSEAVYAGASFRKPVEPY

Mr SDS PAGE : $\quad 56 \mathrm{kDa}$ Identified protein(s) : Enolase (TC112026)

\begin{tabular}{llllll}
\hline exp & cal & ppm & seq & protein & notes \\
\hline 1138.661 & 1138.61 & 45 & TAIEKAGYTGK & &
\end{tabular}




\begin{tabular}{|c|c|c|c|c|}
\hline 1258.613 & 1258.73 & 93 & AGAAVRNVPLYK & \\
\hline 1579.935 & 1579.92 & 12 & AVNNVNSIIGPALIGK & \multirow{7}{*}{$\mathrm{M}$} \\
\hline 1804.927 & 1804.94 & 9 & AAVPSGASTGIYEALELR & \\
\hline 1827.865 & 1827.88 & 6 & IEEELGSEAVYAGASFR & \\
\hline 1919.838 & 1920.01 & 90 & VNQIGSVTESIEAVKMSK & \\
\hline 1951.189 & 1951.13 & 30 & GVSKAVNNVNSIIGPALIGK & \\
\hline 2252.09 & 2252.13 & 17 & SGETEDTFIADLAVGLSTGQIK & \\
\hline 2324.032 & 2324.04 & 5 & YGQDATNVGDEGGFAPNIQENK & \\
\hline 2382.234 & 2382.28 & 18 & LTTEIGEQVQIVGDDLLVTNPK & \multirow{3}{*}{ M } \\
\hline 2406.281 & 2406.20 & 34 & AGYTGKVVIGMDVAASEFYGKDK & \\
\hline 2980.363 & 2980.34 & 7 & SFVSEYPIVSIEDPFDQDDWETYAK & \\
\hline
\end{tabular}

TC112026, Coverage: $46.0 \%(204 / 444)$

MATIKS IKARQ I EDSRGNPTVEVDVHVSNGVFARAAVPSGASTGIYEALELRDGGSDYLGKGVSKAVNNVNSIIGPALIGKDP TDQTGLDNFMVHELDGTQNEWGWCKEKLGANA ILAVSLAVCKAGAAVRNVPLYKH IADLAGNKKLVLPVPAFNVINGGSHAGN KLAMQE FMI LPVGAANFKEAMKMGCEVYHHLKSVI KKKYGQDATNVGDEGGFAPNIQENKEGLELLKTAIEKAGYTGKVVIGM DVAASEFYGKDKSYDLNFKEESNDGSQK I SGDQLKDLYKSFVSEYPIVSIEDPFDQDDWETYAKLTTEIGEQVQIVGDDLLVT NPKRVAKAIAEKTCNALLLKVNQIGSVTESIEAVKMSKKAGWGVMTSHRSGETEDTFIADLAVGLSTGQIKTGAPCRSERLAK YNQLLRIEEELGSEAVYAGASFRKPVEPY

Mr SDS PAGE : $\quad 42 \mathrm{kDa}$ Identified protein(s) : Patatin 2-k1 (Kuras, DQ114415)

\begin{tabular}{|c|c|c|c|c|c|}
\hline $\exp$ & cal & ppm & seq & protein & notes \\
\hline 628.372 & 628.36 & 19 & WGPLR & & \\
\hline 1047.468 & 1047.62 & 144 & TNKPVIFTK & & \\
\hline 1077.712 & 1077.64 & 67 & FAKLLSDRK & & M \\
\hline 1133.526 & 1133.66 & 115 & YLLQVLQEK & & \\
\hline 1272.512 & 1272.68 & 132 & SNLAKSPELDAK & & \\
\hline 1281.592 & 1281.59 & 2 & DSPETYEEALK & & \\
\hline 1305.578 & 1305.67 & 69 & LAQEDPAFSSIK & & \\
\hline 1437.572 & 1437.69 & 79 & DSPETYEEALKR & & \\
\hline 1757.810 & 1757.94 & 76 & VHQALTEVAISSFDIK & & \\
\hline 1853.734 & 1853.93 & 107 & QMLLLSLGTGTNSEFDK & & \\
\hline 1911.972 & 1911.97 & 1 & LAQEDPAFSSIKSLDYK & & \\
\hline 3626.839 & 3626.82 & 5 & LADYFDVIGGTSTGGLLTAMITTPNENNRPFRAAAK & & \\
\hline 3642.839 & 3642.82 & 5 & LADYFDVIGGTSTGGLLTAMITTPNENNRPFRAAAK & & $\mathrm{O}, \mathrm{M}$ \\
\hline
\end{tabular}

DQ114417, Coverage: $38.8 \%(141 / 363)$

TLGEMVTVLS I DGGG I KG I I PAT I LEFLEGQLQEVDNNKDARLADYFDVIGGTSTGGLLTAMITTPNENNRPFAAAKD IVPFY FEHGPHIFNSSGT IFGPMYDGKYLLQVLQEKLGETRVHQALTEVAISSFDIKTNKPVIFTKSNLAKSPELDAKMYDICYSTAA APIYFPPHYFVTHTSNGDRYEFNLVDGAVATVGDPALLSLSVATRLAQEDPAFSS IKSLDYKQMLLLSLGTGTNSEFDKTYTA EEAAKWGPLRWLLAIQQMTNAASSYMTDYYLSTVFQARHSQNNYLRVQENALTGTTTEMDDASEANMELLVQVGETLLKKPVS KDSPETYEEALKRFAKLLSDRKKLRANKASY

Mr SDS PAGE : $\quad 40 \mathrm{kDa}$ Identified protein(s) : Patatin 1-k1 (Kuras, DQ114415) and Patatin 1-k2 (Kuras, DQ114416)

\begin{tabular}{llllll}
\hline exp & cal & ppm & seq & protein & notes \\
\hline 1167.667 & 1167.61 & 49 & YLMQVLQEK & Pat1-k1, Pat1-k2 & O, M \\
1475.773 & 1474.91 & 585 & FAKLLSDRKKLR & Pat1-k2 & Unique, 4 mis \\
1689.900 & 1689.90 & 0 & TLGEMVTVLSIDGGGIK & Pat1-k1 & Unique
\end{tabular}




\begin{tabular}{llllll}
1705.902 & 1705.90 & 1 & TLGEMVTVLSIDGGGIK & Pat1-k1 & O, Unique, M \\
1757.912 & 1757.90 & 7 & VHQALTEVAISSFDIK & Pat1-k1, Pat1-k2 & Pat1-k1 \\
1835.950 & 1835.97 & 11 & QLLLLSLGTGTNSEFDK & Pat1-k2 & Unique \\
2247.156 & 2247.08 & 34 & ADDASEANMELLAQVGENLLK & Pat1-k1 & Unique \\
2441.358 & 2441.28 & 32 & SLNYKQLLLLSLGTGTNSEFDK & Pat1-k2 & 2 O, Unique, M \\
3305.910 & 3305.74 & 51 & VQENALTGTTTKADDASEANMELLAQVGENLLK & & \\
\hline
\end{tabular}

DQ114415, Coverage: 17.6\% (64 / 364)

TLGEMVTVLSIDGGGIKG I I PGI I LEFLEGQLQKMDNNADARLADYFDVIGGTSTGGLLTAMITTPNENNRPFAAAKDIVPFY FQHGPH I FNSSTGQFFGPKYDGKYLMQVLQEKLGETRVHQALTEVAISSFDIKTNKPVIFTKSNLAKSPELDAKMSDICYSTA AAPTYFPPHYFATNTSNGDKYEFNLVDGAVATVADPALLSVSVATRRAEEDPAFAS I RSLNYKQLLLLSLGTGTNSEFDKTHT AQETAKWGALQWMLVIQQMTEAASSYMTDYYLSTVFQDLHSQNNYLRVQENALTGTTTKADDASEANMELLVQVGENLLKKPV SKDNPETYEEALKRFAKLLSDRKKFRANKASY

DQ114416, Coverage: $19.2 \%(70 / 364)$

SLEEMVTVLS I DGGGIKGI I PGTILEFLEGQLQKMDNNADARLADYFDVIGGTSTGGLLTAMITTPNENNRPFAAANEIVPFY FEHGPHIFNSSTGQFFGPKYDGKYLMQVLQEKLGETRVHQALTEVAISSFDIKTNKPVIFTKSNLAKSPELDAKMYDICYSTA AAPTYFPPHYFATNTINGDKYKFNLVDGAVATVADPALLSVSVATRRAQEDPAFAS IRSLNYKKMLLLSLGTGTTSEFDKTHT AEETAKWGALQWMLVIQQMTEAASSYMTDYYLSTVFQDLHSQNNYLRVQENALTGTTTKADDASEANMELLAQVGENLLKKPV SKDNPETYEEALKRFAKLLSDRKKLRANKASY

Mr SDS PAGE : $\quad 25 \mathrm{kDa}$

Identified protein(s) : KPI C-k1 (C-k1, Kuras, BG350049)

KPI C-k2 (C-k2, Kuras, BG350725)

\begin{tabular}{llllll}
\hline exp & cal & ppm & seq & protein & notes \\
\hline 1012.603 & 1012.62 & 17 & LPIPQFLGK & $\mathrm{C}-\mathrm{k} 1$ & Unique \\
1034.605 & 1034.58 & 24 & GTPVMFVRK & $\mathrm{C}-\mathrm{k} 1$ & Unique \\
1056.585 & 1056.55 & 33 & NFAPFVFSK & $\mathrm{C}-\mathrm{k} 2$ & Unique \\
1206.576 & 1206.56 & 13 & KSESDDGDVVR & $\mathrm{C}-\mathrm{k} 2$ & Unique \\
1972.018 & 1972.05 & 16 & IMKTDLVTPEGSKYVYK & $\mathrm{C}-\mathrm{k} 2$ & Unique \\
1983.090 & 1983.05 & 20 & LATVDDDKDFIPFVFIK & $\mathrm{C}-\mathrm{k} 1$ & Unique \\
2262.259 & 2262.09 & 5 & VNDEELVVTGGNVGNENDIFK & $\mathrm{C}-\mathrm{k} 2$ & Unique \\
2544.421 & 2545.33 & 37 & VNDEELVVTGGKVGNENDIFKIR & $\mathrm{C}-\mathrm{k} 1$ & Unique \\
\hline
\end{tabular}

BG350049, Coverage: $31.5 \%(58 / 184)$

LVLPEIYDRDGDPLRI GERYI IENPLIGGGSVYLDN I GNLQCPNAVLQRLPI PQFLGKGTPVMFVRKSESDYGDVVRVMTVVY IKFFVKTTPSCVDETVWKVNDEELVVTGGKVGNENDIFKIRKTDLVIRGMKNVYKLLHCPSHHECKNIGGNFKNGYPRLATVD DDKDFIPEVEIKAKAYNA

BG350725, Coverage: $31.4 \%(58 / 185)$

LVLPEVYDDEGHPLRI GEKYI INNPRLGAGAVYLYNIGNLKCPNAVLQHISTFNLSGKGTPVKFVRKSESDDGDVVRVMTVVY IKFFPNI PNLLCVNENVWKVNDEELVVTGGNVGNENDIFKIMKTDLVTPEGSKYVYKLLHCPAHLECKNIGVNFKDGYPRLTT VDDDKNFAPF'VFSKAKNAN

\section{Mr SDS PAGE : $\quad 24 \mathrm{kDa}$}

Identified protein(s) : KPI A-k1 (A-k1, Kuras, DQ168311)

Represented

KPI A-k2 (A-k2, Kuras, DQ207847)

\begin{tabular}{llllll}
\hline exp & cal & ppm & seq & protein & notes \\
\hline 1105.659 & 1105.56 & 90 & ELNPNLSYR & A-k1 & Unique \\
1112.598 & 1112.57 & 25 & LCVSYTIWK & A-k1, A-k2 & \\
1348.642 & 1348.65 & 6 & YNSDVGPSGTPVR & A-k1, A-k2 &
\end{tabular}




\begin{tabular}{llllll}
1461.683 & 1461.64 & 29 & SPNSDAPCPDGVFR & A-k1, A-k2 & \\
1812.961 & 1812.97 & 5 & LALVNENPLDLFQEV & A-k1, A-k2 & C-term \\
1812.961 & 1812.95 & 6 & IISTYWGALGGDVYLGK & A-k1 & Unique \\
1969.124 & 1969.08 & 22 & RLALVNENPLDVLFQEV & A-k1, A-k2 & M \\
2019.024 & 2018.97 & 27 & TMLLETGGTIGQADSSYFK & A-k1, A-k2 & \\
2146.267 & 2146.15 & 55 & NIFEDQLLNIQFNIPTVK & A-k2 & Unique \\
\hline
\end{tabular}

DQ168311, Coverage: 51.3\% (98/191)

LPSESPLPKPVLDTNGKELNPNLSYRIISTYWGALGGDVYLGKSPNSDAPCPDGVFRYNSDVGPSGTPVRFI PLSTNIFEDQL LNIQFNI PTPKLCVSYTIWKVGN INAP LRTMLLETGGTIGQADSSYFKIVKSSNFGYNLLYCPITRHFLCPFCRDDNFCAKVG VVIQNGKRRLALVNENPLDVLFQEV

DQ207847, Coverage: 47.12\% (90 / 191

LPSES P LPKPVLDTNGKE LNPNSSYRI I I GRGALGGDVYLGKSPNSDAPCPDGVFRYNSDVGPSGTPVRF I PLSKNIFEDQL LNIQFNIPTVKLCVSYTIWKVGNLNAPLWTMLLETGGTIGQADSSYFKIVKS SNFGYNLLYCP ITRHFLCPFCRDDNFCAKVG VVIQNDKRRLALVNENPLDVLFQEV

Mr SDS PAGE : $\quad 20 \mathrm{kDa}$

Identified protein(s) : KPI B-k1 (B-k1, Kuras, DQ168319) KPI B-k2 (B-k2, Kuras, DQ168331)

\begin{tabular}{llllll}
\hline exp & cal & ppm & seq & protein & notes \\
\hline 1034.474 & 1034.52 & 45 & DNPLDVSFK & B-k1, B-k2 & \\
1086.650 & 1086.52 & 120 & SCVSYTIWK & B-k1 & Unique \\
1148.522 & 1148.61 & 77 & ELDPRLSYR & B-k1 & Unique \\
1348.760 & 1348.65 & 81 & YNSDVGPSGTPVR & B-k1, B-k2 & \\
1796.805 & 1796.96 & 86 & IISTFWGALGGDVYLGK & B-k1, B-k2 & B-term \\
1914.078 & 1914.07 & 4 & LALVKDNPLDVSFKQVQ & B-k1, B-k2 & Unique \\
2778.268 & 2778.28 & 4 & SPNSDAPCANGIFRYNSDVGPSGTPVR & B-k2 & Unique \\
3119.860 & 3119.78 & 26 & FIGSSSHFGPHIFEGELLNIQFDISTVK & B-k1 & Unique \\
3259.095 & 3259.62 & 161 & VGDYDASLGTMLLETGGTIGQADSSWFKIVK & B-k2 & \\
\hline
\end{tabular}

DQ168319, Coverage: 48.7\% (93 / 191)

LPSDATPVLDVTGKELDPRLSYRI ISTFWGALGGDVYLGKS PNSDAPCANGVFRYNSDVGPSGTPVRFIGSSSHFGPHIFEGE LLNIQFDISTVKSCVSYTIWKVGDYDAS LGTMLLETGGT I GQEDSSWFKIVKS SQLGYNLLYCPATMICPFCSDDEFCLKVGV IHQNGKRRLALVKDNPLDVSFKQVQ

DQ168331, Coverage: 47.7\% (92/193)

LPSDATPVLDITGKELDSRLSYRI ISTFWGALGGDVYLGKSPNSDAPCANGIFRYNSDVGPSGTPVRFIGSSSHFGQGIFENE LLNIQFA ISTSKLCVSYT IWKVGDYDASLGTMLLETGGTIGQADSSWFKIVKS SQLGYNLLYCPVTSTMICPF SS DDQFCLKV GVVHQNGKRRLALVKDNPLDVSFKQVQ

Mr SDS PAGE : $\quad 18 \mathrm{kDa}$ Identified protein(s) : KPI B-k3 (B-k3, Kuras, DQ268836)

\begin{tabular}{|c|c|c|c|c|c|}
\hline $\exp$ & cal & ppm & seq & protein & notes \\
\hline 1034.449 & 1034.52 & 69 & DNPLDVSFK & & \\
\hline 1112.462 & 1112.57 & 97 & LCVSYTIWK & & \\
\hline 1148.497 & 1148.61 & 98 & ELDPRLSYR & & \\
\hline 1178.443 & 1178.78 & 286 & LSYRIISIGR & & \\
\hline 1332.516 & 1332.65 & 101 & FNSDVGPSGTPVR & & Unique \\
\hline 1914.053 & 1914.07 & 9 & LALVKDNPLDVSFKQVQ & & C-term, $\mathrm{M}$ \\
\hline
\end{tabular}


$\begin{array}{llll}2023.126 & 2023.07 & 28 & \text { LPSDATPVLDVTGKELDPR }\end{array}$

$\begin{array}{llll}3119.835 & 3119.58 & 82 & \text { FIGSSSHFGPHIFEGELLNIQFDISTVK }\end{array}$

DQ268836, Coverage: 51.9\% (96/185)

LPSDATPVLDVTGKELDPRLSYRI ISIGRGALGGDVYLGKS PNSDAPCANGVFRFNSDVGPSGTPVRFIGSSSHFGPHIFEGE LLNIQFDISTVKLCVSYTIWKVGDYDASLGTMLLETGGT I GQADS SWFKIVKS SQLGYNLLYCPFS SDDQFCLKVGVVHQNGK RRLALVKDNPLDVSFKQVQ

Mr SDS PAGE : $\quad 12 \mathrm{kDa}$

Identified protein(s) : Protease inhibitor I PI I-k1 (DQ168322)

\begin{tabular}{llllll}
\hline exp & cal & ppm & seq & protein & notes \\
\hline 1328.712 & 1328.69 & 17 & ESDGPEVIELLK & & \\
1524.891 & 1524.88 & 7 & GKLSWPELIGVPTK & & \\
2150.123 & 2150.06 & 29 & ESDGPEVIELLKEFECKGK & & \\
\hline
\end{tabular}

DQ168322, Coverage: $34.1 \%(31 / 91)$

SFETLMAQKESDGPEVIELLKEFECKGKLSWPELIGVPTKLAKGI IEKQNSL I SNVH I LLNGSPVTLDIRCDRVRLFDNILGA VVQIPVVG

\section{Fraction VII}

Mr SDS PAGE : $\quad 25 \mathrm{kDa}$

Identified protein(s) : KPI A-k1 (A-k1, Kuras, DQ168311)

\begin{tabular}{|c|c|c|c|c|c|}
\hline $\exp$ & cal & ppm & seq & protein & notes \\
\hline 1112.451 & 1112.57 & 56 & LCVSYTIWK & & \\
\hline 1461.536 & 1461.64 & 32 & SPNSDAPCPDGVFR & & \\
\hline 1812.814 & 1812.97 & 67 & LALVNENPLDVLFQEV & & \\
\hline 1812.814 & 1812.95 & 75 & IISTYWGALGGDVYLGK & & Unique \\
\hline 1968.977 & 1969.08 & 23 & RLALVNENPLDVLFQEV & & C-term, M \\
\hline 2018.755 & 2018.97 & 78 & TMLLETGGTIGQADSSYFK & & Unique \\
\hline 2086.955 & 2087.09 & 37 & IVKSSNFGYNLLYCPITR & & \\
\hline 2359.155 & 2359.22 & 3 & TMLLETGGTIGQADSSYFKIVK & & Unique \\
\hline
\end{tabular}

DQ168311, Coverage: 49.2\% (94/191)

LPSESPLPKPVLDTNGKELNPNLSYRIISTYWGALGGDVYLGKSPNSDAPCPDGVFRYNSDVGPSGTPVRFIPLSTNIFEDQL LNIQFN I PT PKLCVSYTIWKVGN INA PLRTMLLETGGTIGQADSSYFKIVKSSNFGYNLLYCPITRHFLCPFCRDDNFCAKVG VVIQNGKRRLALVNENPLDVLFQEV

Mr SDS PAGE : $\quad 24 \mathrm{kDa}$

Identified protein(s) : KPI A-k1 (A-k1, Kuras, DQ168311)

KPI A-k2 (A-k2, Kuras, DQ207847)

KPI B-k1 (B-k1, Kuras, DQ168319)

KPI B-k2 (B-k2, Kuras, DQ168331)

KPI B-k4 (B-k4, TC111942)

KPI C-k1 (C-k1, Kuras, BG350049)

KPI C-k2 (C-k2, Kuras, BG350725)

KPI C-k3 (C-k3, Kuras, BG351756)

KPI C-k4 (C-k4, TC119013)

KPI K-k1 (K-k1, TC112888) 
KPI M-k1 (M-k1, TC112274)

KPI M-k2 (M-k2, TC112554)

\begin{tabular}{|c|c|c|c|c|c|}
\hline $\exp$ & cal & ppm & seq & protein & notes \\
\hline 1012.493 & 1012.62 & 125 & LPIPQFLGK & C-k1 & Unique \\
\hline 1034.495 & 1034.52 & 24 & DNPLDVSFK & B-k1, B-k2, B-k4 & \\
\hline 1034.495 & 1034.58 & 82 & GTPVMFVRK & C-k1 & \\
\hline 1056.475 & 1056.55 & 71 & NFAPFVFSK & C-k2 & Unique \\
\hline 1059.411 & 1059.58 & 159 & DTNIGIKSIV & M-k1 & Unique, C-term \\
\hline 1085.671 & 1085.52 & 139 & SCVSYTIWK & B-k1 & Unique \\
\hline 1092.514 & 1092.58 & 60 & NVASWFQIK & M-k1 & Unique \\
\hline 1105.569 & 1105.56 & 8 & ELNPNLSYR & A-k1 & Unique \\
\hline 1112.508 & 1112.57 & 56 & LCVSYTIWK & A-k1, A-k2, B-k2, B-k4 & \\
\hline 1148.543 & 1148.61 & 58 & ELDPRLSYR & B-k1, B-k4 & \\
\hline 1177.489 & 1177.78 & 247 & LSYRIISIGR & B-k4 & \\
\hline 1206.466 & 1206.56 & 78 & KSESDDGDVVR & $\mathrm{C}-\mathrm{k} 2$ & Unique \\
\hline 1286.619 & 1286.77 & 117 & GMPVIFKPKAAK & M-k1 & Unique \\
\hline 1348.781 & 1348.65 & 97 & YNSDVGPSGTPVR & $\begin{array}{l}\text { A-k1, A-k2, B-k1, B-k2, } \\
\text { B-k4 }\end{array}$ & \\
\hline 1421.673 & 1421.74 & 47 & TTKLCVDQTVWK & $\mathrm{C}-\mathrm{k} 4$ & Unique \\
\hline 1429.617 & 1429.87 & 177 & IRKTDLVIRGMK & C-k1 & Unique \\
\hline 1461.593 & 1461.64 & 32 & SPNSDAPCPDGVFR & A-k1, A-k2 & \\
\hline 1609.756 & 1609.85 & 58 & YFIVSAIWGAGGGGVR & $\mathrm{M}-\mathrm{k} 2$ & Unique \\
\hline 1711.758 & 1711.98 & 130 & KTDLVTPEGSKFVYK & $\mathrm{C}-\mathrm{k} 4$ & Unique \\
\hline 1723.761 & 1723.92 & 92 & AFVFQKDERIGMAIV & M-k2 & Unique, $\mathrm{C}$-term \\
\hline 1796.826 & 1796.96 & 75 & IISTFWGALGGDVYLGK & B-k1, B-k2 & \\
\hline 1812.814 & 1812.97 & 67 & LALVNENPLDVLFQEV & A-k1, A-k2 & C-term \\
\hline 1812.814 & 1812.95 & 75 & IISTYWGALGGDVYLGK & A-k1 & Unique \\
\hline 1834.872 & 1834.78 & 50 & DLYRGMPVIFKPKAAK & M-k1 & Unique \\
\hline 1914.099 & 1914.07 & 15 & LALVKDNPLDVSFKQVQ & B-k1, B-k2, B-k4 & C-term \\
\hline 1967.192 & 1967.03 & 82 & NSRYFIVSAIWGAGGGVR & M-k2 & Unique \\
\hline 1969.034 & 1969.08 & 23 & RLALVNENPLDVLFQEV & A-k1, A-k2 & C-term \\
\hline 1971.908 & 1972.05 & 72 & IMKTDLVTPEGSKYVYK & $\mathrm{C}-\mathrm{k} 2$ & Unique \\
\hline 1982.98 & 1983.05 & 35 & LATVDDDKDFIPFVFIK & C-k1 & Unique \\
\hline 2010.974 & 2011.04 & 33 & LVTVDDDQDFIPFVFIK & $\mathrm{C}-\mathrm{k} 3$ & Unique \\
\hline 2010.974 & 2011.08 & 53 & LVTVDDDKDFLPFVFIK & C-k4 & Unique \\
\hline 2023.172 & 2023.07 & 50 & LPSDATPVLDVTGKELDPR & B-k1, B-k4 & N-term \\
\hline 2109.04 & 2109.06 & 9 & LLGYELITCDGALVGTMGQR & K-k1 & Unique \\
\hline 2146.177 & 2146.15 & 13 & NIFEDQLLNIQFNIPTVK & A-k2 & Unique \\
\hline 2146.177 & 2146.09 & 41 & KSESDYGDVVRVMTGVYIK & $\mathrm{C}-\mathrm{k} 3, \mathrm{C}-\mathrm{k} 4$ & \\
\hline 2226.131 & 2226.12 & 5 & VNHEGLVVTGGQVGNENDIFK & $\mathrm{C}-\mathrm{k} 4$ & Unique \\
\hline 2262.149 & 2262.09 & 26 & VNDEELVVTGGNVGNENDIFK & $\mathrm{C}-\mathrm{k} 2$ & \\
\hline 2545.511 & 2545.33 & 70 & VNDEELVVTGGKVGNENDIFKIR & C-k1 & Unique \\
\hline 2546.311 & 2546.32 & 4 & FVYKLLHCPSHLQCKNIGGNFK & $\mathrm{C}-\mathrm{k} 3, \mathrm{C}-\mathrm{k} 4$ & \\
\hline 2570.343 & 2571.33 & 5 & NGYPRLATVDDDKDFIPFVFIK & C-k1 & Unique \\
\hline 2759.311 & 2759.26 & 18 & RGPYGGDIYLDYSPGSTAPCPDGVFR & K-k1 & Unique \\
\hline 2778.289 & 2778.65 & 130 & SPNSDAPCANGIFRYNSDVGPSGTPVR & B-k2 & Unique \\
\hline 3119.881 & 3119.78 & 32 & FIGSSSHFGPHIFEGELLNIQFDISTVK & B-k1 & \\
\hline 3121.671 & 3121.56 & 36 & FIGSSSHFGPHIFEDELLNIQFAISTSK & B-k4 & Unique \\
\hline 3259.116 & 3259.235 & 37 & VGDYDASLGTMLLETGGTIGQADSSWFKIVK & B-k2, B-k4 & \\
\hline
\end{tabular}

DQ168311, Coverage: 41.4\% (79/ 191) 
LPSESPLPKPVLDTNGKELNPNLSYRIISTYWGALGGDVYLGKSPNSDAPCPDGVFRYNSDVGPSGTPVRF I PLSTNI FEDQL LNIQFNI PTPKLCVSYTIWKVGN INAPLRTMLLETGGTIGQADSSYFKIVKS SNFGYNLLYCP ITRHFLCPFCRDDNFCAKVG VVIQNGKRRLALVNENPLDVLFQEV

DQ207847, Coverage: 37.2\% (71/191)

LPSES P LPKPVLDTNGKE LNPNSSYR I I I GRGALGGDVYLGKSPNSDAPCPDGVFRYNSDVGPSGTPVRF I PLSKNIFEDQL LNIQFNIPTVKLCVSYTIWKVGNLNAPLWTMLLETGGTIGQADSSYFK IVKS SNFGYNLLYCP ITRHF LCPFCRDDNFCAKVG VVIQNDKRRLALVNENPLDVLFQEV

DQ168319, Coverage: 56.0\% (107/ 191)

LPSDATPVLDVTGKELDPRLSYRI ISTFWGALGGDVYLGKS PNSDAPCANGVFRYNSDVGPSGTPVRFIGSSSHFGPHIFEGE LLNIQFDISTVKSCVSYTIWKVGDYDASLGTMLLETGGT I GQEDS SWFKIVKS SQLGYNLLYCPATMICPFCSDDEFCLKVGV IHQNGKRRLALVKDNPLDVSFKQVQ

DQ168331, Coverage: 52.3\% (101/193)

LPSDATPVLDITGKELDSRLSYRI ISTFWGALGGDVYLGKSPNSDAPCANGIFRYNSDVGPSGTPVRFIGSSSHFGQGIFENE LLNIQFA ISTSKLCVSYTIWKVGDYDASLGTMLLETGGTIGQADSSWFKIVKSSQLGYNLLYCPVTS TMICPFSSDDQFCLKV GVVHQNGKRRLALVKDNPLDVSFKQVQ

TC111942, Coverage: $68.7 \%(127 / 185)$

LPSDATPVLDVTGKELDPRLSYRI ISIGRGALGGDVYLGKS PNSDAPCANGVFRYNSDVGPSGTPVRFIGSSSHFGPHIFEDE LLNIQFAISTSKLCVSYTIWKVGDYDASLGTMLLETGGTIGQADSSWFKIVKS SQLGYNLLYCPF S SDDQFCLKVGVVHQNGK RRLALVKDNPLDVSFKQVQ

BG350049, Coverage: 39.7\% (73/184)

LVLPEIYDRDGDPLRI GERYI IENPLIGGGSVYLDNIGNLQCPNAVLQRLPIPQFLGKGTPVMFVRKSESDYGDVVRVMTVVY IKFFVKTTPSCVDETVWKVNDEELVVTGGKVGNENDIFKIRKTDLVIRGMKNVYKLLHCPSHHECKNIGGNFKNGYPRLATVD DDKDFIPFVFIKAKAYNA

BG350725, Coverage: $31.4 \%(58 / 185)$

LVLPEVYDDEGHPLRI GEKYI INNPRLGAGAVYLYNIGNLKCPNAVLQHISTFNLSGKGTPVKFVRKSESDDGDVVRVMTVVY IKFFPNI PNLLCVNENVWKVNDEELVVTGGNVGNENDIFKIMKTDLVTPEGSKYVYKLLHCPAHLECKNIGVNFKDGYPRLTT VDDDKNFAPFVFSKAKNAN

BG351756, Coverage: $32.4 \%(58 / 179)$

LVLPEVYDQDGHPLRI GERYI INNPLIGAGAVYLYNIGNLQCPNAVLQHMS I PQFLGEGTPVKFVRKSESDYGDVVRVMTGVY IKFFVKPTKLCVDQTVWKVNDEGLVVTGGKVGNENDIFKIRKTDLVTPGGSKFVYKLLHCPSHLQCKNIGGNFKNGYPRLVTV DDDQDFIPFVFIK

TC119013, Coverage: 56.7\% (102/180)

LVLPEVYDQNGHPLRIGQRYI INNPLIGAGAVYLYNIGNLQCPNAVLQHMS I PQFLGEGTPVVFVRKSESDYGDVVRVMTGVY IKFFVKTTKLCVDQTVWKVNHEGLVVTGGQVGNENDIFKIRKTDLVTPEGSKFVYKLLHCPSHLQCKNIGGNFKNGY PRLVTV DDDKDFLPFVFIKA

TC112888, Coverage: $25.3 \%(46 / 182)$

STFTSQNPIDLPSAKSVPVLDTTGKEVDPRWSYRMVYTKRGPYGGDIYLDYSPGSTAPCPDGVFRYGQVGPKGTPVRLITPSH FGPGVYVDQE INIQFVI SNVEKCGSYT IWKVGPYDNEDRVSFLETGQQNSKSCFKIVKS PRLLGYELITCDGALVGTMGQRVA LVSNFSLDFEFEKVED

TC112274, Coverage: $17.5 \%(35 / 200)$

SCTSATTTPNPVLQVVRDI HGDILTPDSRYFVVSAITGAGGGGVFRGIGAGHDANFVCPFQVLQSGRDLYRGMPVIFKPKAAK QVEITESSDVNIEFY IDNPSGICNNTVWEVEGFPGHDMPMYLATNGEAGHVKNVASWFQIKKTGSYMYKLMFCPYGEPICTDI GIDYTAGRRLAIGTGNTFNLVFIKDTNIGIKSIV

TC112554, Coverage: $15.6 \%(34 / 217)$ 
MKI I LLLLFS LAFLLLFTLAS STNNI PNQAFRT IRDIEGNPLNKNSRYFIVSAIWGAGGGGVRLANLGNQGQNDCPTSVVQSH NDLDNGIAVYITPHDPKYDIISEMSTVNIKFYLDSPTCSHFTMWMVNDFPKPADQLYTISTGEQLIDSVNLNNRFQIKSLGGS TYKLVFCPYGEKFTCQNVGIADENGYNRLVLTENEKAFVFQKDERIGMAIV

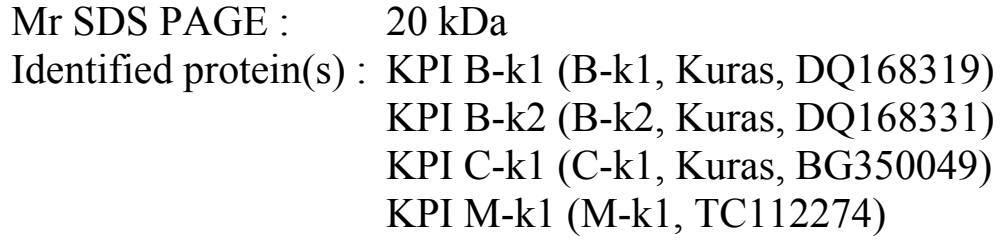

\begin{tabular}{|c|c|c|c|c|c|}
\hline $\exp$ & cal & ppm & seq & protein & notes \\
\hline 1012.572 & 1012.62 & 48 & LPIPQFLGK & C-k1 & Unique \\
\hline 1034.574 & 1034.52 & 52 & DNPLDVSFK & B-k1, B-k2 & \\
\hline 1059.782 & 1059.58 & 190 & DTNIGIKSIV & M-k1 & Unique, C-term \\
\hline 1085.750 & 1085.52 & 212 & SCVSYTIWK & B-k1 & Unique \\
\hline 1092.593 & 1092.58 & 12 & NVASWFQOK & M-k1 & Unique \\
\hline 1112.587 & 1112.57 & 15 & LCVSYTIWK & B-k2 & \\
\hline 1148.622 & 1148.61 & 10 & ELDPRLSYR & B-k1 & \\
\hline 1286.698 & 1286.77 & 56 & GMPVIFKPKAAK & M-k1 & Unique \\
\hline 1348.860 & 1348.65 & 156 & YNSDVGPSGTPVR & B-k1, B-k2 & \\
\hline 1429.696 & 1429.87 & 122 & IRKTDLVIRGMK & C-k1 & Unique \\
\hline 1796.905 & 1796.96 & 31 & IISTFWGALGGDVYLGK & B-k1, B-k2 & \\
\hline 1834.951 & 1834.78 & 93 & DLYRGMPVIFKPKAAK & M-k1 & Unique \\
\hline 1914.178 & 1914.07 & 56 & LALVKDNPLDVSFKQVQ & B-k1, B-k2 & C-term \\
\hline 1983.059 & 1983.05 & 4 & LATVDDDKDFIPFVFIK & C-k1 & Unique \\
\hline 2571.422 & 2571.33 & 36 & NGYPRLATVDDDKDFIPFVFIK & C-k1 & Unique \\
\hline 2778.368 & 2778.28 & 32 & SPNSDAPCANGIFRYNSDVGPSGTPVR & B-k2 & Unique \\
\hline 3119.960 & 3119.78 & 58 & FIGSSSHFGPHIFEGELLNIQFDISTVK & B-k1 & \\
\hline 3259.195 & 3259.24 & 12 & VGDYDASLGTMLLETGGTIGQADSSWFKIVK & B-k2 & \\
\hline
\end{tabular}

DQ168319, Coverage: 48.7\% (93/191)

LPSDATPVLDVTGKELDPRLSYRIISTFWGALGGDVYLGKS PNSDAPCANGVFRYNSDVGPSGTPVRFIGSSSHFGPHIFEGE LLNIQFDISTVKSCVSYTIWKVGDYDAS LGTMLLETGGT I GQEDSSWFKIVKSSQLGYNLLYCPATMICPFCSDDEFCLKVGV IHQNGKRRLALVKDNPLDVSFKQVQ

DQ168331, Coverage: 52.3\% (101/193)

LPSDATPVLDITGKELDSRLSYRIISTFWGALGGDVYLGKSPNSDAPCANGIFRYNSDVGPSGTPVRFIGSSSHFGQGIFENE LLNIQFA ISTSKLCVSYTIWKVGDYDASLGTMLLETGGTIGQADSSWFKIVKS SQLGYNLLYCPVTS TMICPFSSDDQFCLKV GVVHQNGKRRLALVKDNPLDVSFKQVQ

BG350049, Coverage: $23.4 \%(43 / 184)$

LVLPEIYDRDGDPLRI GERYI IENPLIGGGSVYLDNIGNLQCPNAVLQRLPIPQFLGKGTPVMFVRKSESDYGDVVRVMTVVY IKFFVKTTPSCVDETVWKVNDEELVVTGGKVGNENDIFKIRKTDLVIRGMKNVYKLLHCPS HHECKNIGGNFKNGYPRLATVD DDKDFIPFVFIKAKAYNA

TC112274, Coverage: 17.5\% (35/200)

SCTSATTTPNPVLQVVRDIHGDILTPDSRYFVVSAITGAGGGGVFRG IGAGHDANFVCPFQVLQSGRDLYRGMPVIFKPKAAK QVEITESSDVNIEFYIDNPSGICNNTVWEVEGFPGHDMPMYLATNGEAGHVKNVASWFQIKKTGSYMYKLMFCPYGEPICTDI GIDYTAGRRLAIGTGNTFNLVFIKDTNIGIKSIV

\section{Fraction VIII}

Mr SDS PAGE : $\quad 25 \mathrm{kDa}$ 
Identified protein(s) : KPI C-k4 (TC112013)

Present : $\quad$ KPI C-k1 (Kuras, BG350049)

\begin{tabular}{llllll}
\hline exp & cal & ppm & seq & protein & notes \\
\hline 1012.414 & 1012.62 & 24 & LPIPQFLGK & $\mathrm{C}-\mathrm{k} 1$ & Unique \\
1421.594 & 1421.74 & 103 & TTKLCVDQTVWK & $\mathrm{C}-\mathrm{k} 4$ & Unique \\
1429.538 & 1429.87 & 232 & IRKTDLVIRGMK & $\mathrm{C}-\mathrm{k} 1$ & Unique \\
1711.679 & 1711.98 & 176 & KTDLVTPEGSKFVYK & $\mathrm{C}-\mathrm{k} 4$ & Unique \\
1982.901 & 1983.05 & 75 & LATVDDDKDFIPFVFIK & $\mathrm{C}-\mathrm{k} 1$ & Unique \\
2010.895 & 2011.08 & 92 & LVTVDDDKDFLPFVFIK & $\mathrm{C}-\mathrm{k} 4$ & Unique \\
2226.052 & 2226.12 & 31 & VNHEGLVVTGGQVGNENDIFK & $\mathrm{C}-\mathrm{k} 4$ & Unique \\
2545.681 & 2545.33 & 138 & VNDEELVVTGGKVGNENDIFKIR & $\mathrm{C}-\mathrm{k} 1$ & Unique \\
2546.719 & 2546.32 & 157 & FVYKLLHCPSHLQCKNIGGNFK & $\mathrm{C}-\mathrm{k} 4$ & \\
2571.264 & 2571.33 & 26 & NGYPRLATVDDDKDFIPFVFIK & $\mathrm{C}-\mathrm{k} 1$ & Unique \\
3242.747 & 3242.68 & 21 & YIIENPLIGGGSVYLDNIGNLQCPNAVLQR & $\mathrm{C}-\mathrm{k} 1$ & Unique \\
\hline
\end{tabular}

TC112013, Coverage: 46.1\% (83/180)

LVLPEVYDQNGHPLR IGQRYI INNPLIGAGAVYLYN I GNLQCPNAVLQHMS I PQFLGEGTPVVFVRKSESDYGDVVRVMTGVY IKFFVKTTKLCVDQTVWKVNHEGLVVTGGQVGNENDIFKIRKTDLVTPEGSKFVYKLLHCPSHLQCKNIGGNFKNGYPRLVTV DDDKDFLPFVFIKA

BG350049, Coverage: 51.1\% (94 / 184)

LVLPEIYDRDGDPLRIGERYIIENPLIGGGSVYLDNIGNLQCPNAVLQRLPI PQFLGKGTPVMFVRKSESDYGDVVRVMTVVY IKFFVKTTPSCVDETVWKVNDEELVVTGGKVGNENDIFKIRKTDLVIRGMKNVYKLLHCP SHHECKNIGGNFKNGYPRLATVD DDKDFIPFVFIKAKAYNA

Mr SDS PAGE : $22 \mathrm{kDa}$

Identified protein(s) : KPI C-k7 (Kuras, K1-01898)

\begin{tabular}{|c|c|c|c|c|c|}
\hline $\exp$ & cal & ppm & seq & protein & notes \\
\hline 1018.612 & 1018.57 & 41 & TDLVVRGMK & & Unique \\
\hline 1225.671 & 1225.58 & 74 & SESDYRDVVR & & Unique \\
\hline 1379.779 & 1379.77 & 7 & DVVRVMTGVYIK & & Unique \\
\hline 2033.263 & 2033.11 & 75 & LVTVHDDKDFIPFVFIK & & Unique, C-term \\
\hline 2038.991 & 2039.02 & 14 & LLHCPSHLQCKNIGSNFK & & Unique \\
\hline
\end{tabular}

K1-01898, Coverage: $34.8 \%(62 / 178)$

LVLPEVYDQDGNPLRI GERYI I KNPLLGGGAVYLDNI GNLQCPNAVLQHMS I PQFLGKGTPVMFVRKSESDYRDVVRVMTGVY IKFFVKTSRLCVDETVWKVNHEQLVVTGGNVGNENDIFKIKKTDLVVRGMKNVYKLLHCPSHLQCKNIGSNFKNGYPRLVTVH DDKDFIPFVFIK

Mr SDS PAGE : $\quad 8 \mathrm{kDa}$ Identified protein(s) : Carboxypeptidase inhibitor (CPI-k2, TC119261)

\begin{tabular}{llllll}
\hline exp & cal & ppm & seq & protein & notes \\
\hline 696.591 & 696.39 & 288 & TCGPYVG & C-term \\
1742.129 & 1741.85 & 169 & LFQQHADPICNKPCK & N-term \\
\hline
\end{tabular}

TC126340, Coverage: $53.7 \%(22 / 41)$

LFQQHADPICNKPCKTHDDCSGAWFCQACWNSARTCGPYVG 